Rivista dell'Associazione Italiana Financial Industry Risk Managers

Anno 13, numero 3

Settembre - Dicembre 2018

Poste Italiane - Spedizione in abbonamento postale - 70\% aut. DCB / Genova nr. 569 anno 2005

TESTATA INDIPENDENTE CHE NON PERCEPISCE CONTRIBUTI PUBBLICI (legge 250/1990)

\section{IN QUESTO NUMERO}

\section{ARTICOLI A CARATTERE DIVULGATIVO}

\begin{tabular}{|l|l|}
\hline 3 & $\begin{array}{l}\text { Banche, il business model che verrà } \\
\text { di Maurizio Pierigè }\end{array}$ \\
\hline
\end{tabular}

\section{ARTICOLI A CARATTERE SCIENTIFICO} (sottoposti a referaggio)

\begin{tabular}{|c|c|}
\hline 11 & $\begin{array}{l}\text { La misurazione dell'esposizione al rischio } \\
\text { di tasso di interesse del portafoglio } \\
\text { bancario: quali implicazioni in sede ICAAP } \\
\text { a seguito della recente introduzione } \\
\text { dell'approccio del margine di interesse nel } \\
\text { quadro normativo di vigilanza prudenziale? } \\
\text { di Igor Gianfrancesco }\end{array}$ \\
\hline 37 & $\begin{array}{l}\text { Confronto tra l'approccio tradizionale e le } \\
\text { tecniche di Machine Learning per la } \\
\text { modellizzazione della stagionalità nella } \\
\text { valorizzazione degli swap indicizzati } \\
\text { all'inflazione }\end{array}$ \\
\hline & di Ottavio Caligaris e Pier Giuseppe Giribone \\
\hline
\end{tabular}

Risk Management Magazine

Anno 13, n 3 Settembre - Dicembre 2018

Direttore Responsabile:

Maurizio Vallino

\section{Condirettore}

Corrado Meglio

\section{Consiglio scientifico}

Giampaolo Gabbi (Direttore del Consiglio Scientifico), Ruggero Bertelli, Paola Bongini, Anna Bottasso, Marina Brogi, Ottavio Caligaris, Rosita Cocozza, Simona Cosma, Paola Ferretti, Andrea Giacomelli, Adele Grassi, Pier Giuseppe Giribone, Valentina Lagasio, Duccio Martelli, Laura Nieri, Pasqualina Porretta, Anna Grazia Quaranta, Francesco Saita, Enzo Scannella, Cristiana Schena, Giuseppe Torluccio.

Comitato di redazione

Sara Brianza, Emanuele Diquattro, Fausto Galmarini, Igor Gianfrancesco, Rossano Giuppa, Aldo Letizia, Paolo Palliola, Enzo Rocca, Fabio Salis

Vignettista: Silvano Gaggero

Proprietà, Redazione e Segreteria:

Associazione Italiana Financial Industry Risk Managers (AIFIRM), Via Sile 18, 20139 Milano

Registrazione del Tribunale di Milano $n^{\circ} 629$ del 10/9/2004 ISSN 2283-7329

E-mail: amministrazione@ aifirm.it; Tel. 3896946315

Stampa: Algraphy S.n.c. - Passo Ponte Carrega 62-62r 16141 Genova

Le opinioni espresse negli articoli impegnano unicamente la responsabilità dei rispettivi autori

SPEDIZIONE IN ABBONAMENTO POSTALE AI SOCI AIFIRM RESIDENTI IN ITALIA, IN REGOLA CON L'ISCRIZIONE

Rivista in stampa il 20 Dicembre 2018 


\section{Processo di referaggio degli articoli proposti per la pubblicazione}

Gli articoli che sono proposti alla rivista per la pubblicazione sono sottoposti in forma anonima a due successivi livelli di referaggio.

Il primo livello di referaggio (di ammissibilità) viene effettuato sull'articolo dai membri del Consiglio Scientifico che ne valutano la congruità ai temi trattati dalla rivista.

Il secondo livello di referaggio (di pubblicabilità) viene effettuato sull'articolo da due referee scelti all'interno del Consiglio Scientifico o all'esterno tra accademici, ricercatori, esperti della materia, che ne valutano il contenuto e forma.

\section{Regolamento redazionale}

"Risk Management Magazine" è il periodico di AIFIRM (Associazione Italiana Financial Industry Risk Managers) ed interamente dedicato ai temi del risk management.

La sua struttura organizzativa prevede, oltre al direttore responsabile, un condirettore e un Consiglio Scientifico formato da accademici; quest'ultimo è garante della qualità e correttezza degli articoli pubblicati.

La rivista favorisce la diffusione di tutti i contenuti afferenti i temi del risk management, dagli aspetti normativi a quelli organizzativi e alle technicalities e vaglierà con interesse, per mezzo del Comitato Scientifico, i contributi che le perverranno.

Gli articoli proposti dovranno pervenire in formato Microsoft Word carattere Times New Roman 10 ed avere un numero di battute compreso tra 6.000 e 20.000; è gradita la presenza di tabelle e grafici.

La bibliografia deve essere redatta specificando accuratamente le fonti.

Si richiede la predisposizione di un Abstract in lingua inglese.

Le opinioni espresse negli articoli impegnano unicamente la responsabilità dei rispettivi autori.

Sul sito $\underline{w w w . a i f i r m . i t ~ e ̀ ~ p u b b l i c a t a ~ l a ~ D i c h i a r a z i o n e ~ s u l l ' e t i c a ~ e ~ s u l l e ~ p r a t i c h e ~ s c o r r e t t e ~}$ nella pubblicazione dei lavori scientifici.

\section{Referee del 2018}

Si ringraziano i referee sottoelencati che hanno collaborato con la rivista nel 2018: Anna Bottasso, Marina Brogi, Ottavio Caligaris, Simona Cosma, Andrea Giacomelli, Pier Giuseppe Giribone, Duccio Martelli, Laura Nieri, Pasqualina Porretta, Grazia Quaranta, Enzo Scannella, Cristiana Schena, Giuseppe Torluccio. 


\title{
Banche, il business model che verrà
}

\author{
di Maurizio Pierigè (Prometeia)
}

\section{Abstract}

The "Business model issue" is a topic of growing interest for the whole financial community as well as for banking regulators. As we know it today, the business model is a complex and dynamic object, composed of multiple elements and characterized by different facets that make it challenging to just give a shared definition and identify an appropriate analytical approach for its assessment.

It's also still rapidly changing due to a series of factors including the disruptive digital innovation, the increasing regulatory pressures and the gradual exit from the financial crisis that has forced banks to a long period of low profitability. So the financial intermediaries are frequently involved into strategic rethinking on the viability and sustainability of their business models and on evaluating business transformations that could also be radical.

How far will the banks push themselves on this path? What impacts these changes will reshape the structure of the financial sector? What characteristics will have the winning competitors? It is not easy to answer these questions; maybe today we can just guess the scope of change, but we do not fully understand its consequences and implications yet.

The following paper tries to define business models, summarize the analytical approach both from a management and regulatory point of view and imagine their possible transformation according to the changes in the competitive environment.

\section{Premessa}

L'espressione "business model" è probabilmente tra quelle che ricorrono maggiormente - e che suscitano maggiore interesse - nel confronto, istituzionale o meno, tra esponenti della comunità finanziaria e matter expert. Vista la diretta interrelazione con i meccanismi di generazione della redditività, è senza dubbio un tema la cui crescente rilevanza è giustificata dal lungo periodo di debolezza del sistema bancario italiano, con la maggior parte degli istituti in difficoltà nel generare flussi di utili in grado di remunerare il costo del capitale.

Il presidio del business model è quindi divenuto un tema (o una preoccupazione) ricorrente tra i regolatori del sistema bancario europeo, che lo hanno posto al centro dell'attenzione inserendolo tra le "ECB annual priority" a partire dal 2016 . Nel corso del 2017-2018, poi, la BCE ha lanciato specifiche iniziative, come la "Thematic review on profitability", finalizzate ad indagare lo stato di salute del sistema bancario su alcuni elementi specifici del modello di business ed eventuali criticità riscontrate dai singoli operatori significant, rafforzando in parallelo il livello di controllo esercitato sui business model nell'ambito del processo SREP, attraverso un potenziamento delle analisi condotte in sede di Business Model Analysis (BMA).

L'innovazione del business model è però un tema con cui i CEO bancari hanno necessariamente iniziato a confrontarsi, anche in modo disgiunto dalle aspettative della Vigilanza, dato l'avvio del processo di digitalizzazione del sistema finanziario, che sta radicalmente mutando il contesto competitivo di riferimento (nuovo contesto normativo nei sistemi di incasso e pagamento e nei servizi di investimento, nuovi operatori, nuovi competitor, etc.) e il modo di fare banca, attraverso un nuovo business mix (prodotti-clienti-canali).

Molte banche hanno inserito nei propri piani industriali una serie di iniziative finalizzate a introdurre rilevanti innovazioni tecnologiche che stanno progressivamente trasformando il modo con cui organizzano la propria catena del valore: in particolare, la catena produzione-distribuzione-gestione del post vendita. Questo processo di transizione appare tuttavia tuttora fluido e in continua evoluzione: molte banche hanno "aggiunto" iniziative spot, mentre alcune hanno anche sviluppato una strategia digitale con obiettivi chiari e investimenti rilevanti.

Ma quando si può dire che la banca ha effettivamente cambiato il proprio business model? E se lo cambia troppo, ad esempio focalizzandosi su una sola base della catena del valore, sarà ancora una "banca"?

Alcuni operatori hanno maturato una riflessione di tale portata da implicare una revisione del proprio modello di business così intensa da riconsiderare il proprio posizionamento all'interno dell'ecosistema finanziario, o forse addirittura da uscirne.

Alcune esperienze di banche europee sembrano suggerire che il processo di transizione possa, almeno in alcuni casi, concludersi infatti con una radicale revisione del ruolo delle banche commerciali. In quale direzione e con quali tempistiche non è ancora chiaro.

Questo articolo intende fornire qualche spunto di riflessione per inquadrare lo stato dell'arte sul tema dei business model e immaginare una possibile direttrice di evoluzione futura. Nella misura in cui si affermeranno nuovi modelli, anche i tradizionali paradigmi di valutazione dei rischi dovranno cambiare.

\section{Cos'è un business model?}

Innanzitutto, il termine "business model" è spesso utilizzato per associare concetti simili ma non necessariamente unanimemente condivisi, con il rischio di incorrere in equivoci e incomprensioni. Può quindi essere utile uno sforzo definitorio a partire dai contributi offerti dalla letteratura.

La letteratura sui business model è infatti relativamente ampia e offre diverse definizioni. Tra le numerose disponibili, secondo A. Osterwalder "il business model descrive la logica con la quale un'organizzazione crea, distribuisce e cattura valore". In altre parole, è la struttura logica a monte che definisce le relazioni e il comportamento di ogni singolo elemento e che permette all'impresa di lavorare in modo fluido, ottimizzato e produttivo. 
Per creare valore, un'azienda deve comprendere in modo approfondito il proprio business model. Questo significa innanzitutto conoscerne il perimetro di riferimento, le componenti e il sistema di interrelazioni (culturali, funzionali ed organizzative) che lo caratterizzano: è solo attraverso un'analisi accurata del business model che diventa possibile identificare le fonti di generazione del valore dell'impresa e indirizzare le azioni strategiche necessarie a garantire il loro sviluppo e/o a garantire il loro arricchimento con ulteriori combinazioni profittevoli.

Per supportare la mappatura e la comprensione del modello di business, può risultare utile utilizzare il modello "canvas" (cfr. Business Model Generation, edizioni FAG) che, attraverso una rappresentazione grafica, consente di rappresentare in modo sintetico (ma esaustivo) le principali caratteristiche/componenti endogene del business model.

\begin{tabular}{|l|l|l|l|l|}
\hline \multirow{2}{*}{ Partner chiave } & Attività chiave & Proposte di & Relazioni con i clienti & Segmenti di clientela \\
\cline { 2 - 3 } & Ralore & Ranali & \\
\hline Struttura dei costi & Struttura dei ricavi & \\
\hline
\end{tabular}

- Partner chiave;

- Attività chiave;

- Risorse chiave;

- Relazione con i clienti;

- Segmenti di clientela;

- Canali;

- Proposte di valore;

- Struttura dei costi;

- Struttura dei ricavi.

Mappare le componenti principali del modello di business non è però di per sé sufficiente per una sua piena comprensione. L'identificazione del posizionamento a tendere rispetto a ciascuna delle suddette componenti attraverso la valutazione di:

- $\quad$ contesto di mercato;

- contesto regolamentare;

- contesto socio-culturale;

può infatti contribuire in modo significativo alla definizione del proprio modello di business e alla adozione di politiche di gestione ad esso pienamente coerenti.

\section{La Business Model Analysis nel processo SREP}

Con il faro acceso dal regolatore su redditività e business model, la tematica è uscita dal "perimetro" dei piani industriali dove le banche dichiarano la loro mission e/o value proposition e/o business idea (a volte coprendo solo alcune componenti del modello canvas) ed è entrata nell'agenda del CRO, richiedendo una definizione chiara di ruoli e responsabilità, in particolare tra area Risk e Finance.

L'obiettivo della Business Model Analysis (c.d. BMA) è valutare, attraverso un assessment qualitativo e uno quantitativo, due principali aspetti del modello imprenditoriale e strategico attraverso cui la banca si muove sul mercato. Cioè:

- la solidità del modello di business corrente della banca: capacità di generare utili adeguati su un orizzonte temporale di breve termine (12 mesi);

- la sostenibilità dei piani strategici: capacità di generare, a partire dai piani strategici e dalle previsioni economicofinanziarie, utili adeguati su un orizzonte temporale di lungo termine (almeno 3 anni).

Nelle intenzioni del regolatore, la BMA dovrebbe pertanto supportare la comprensione della profittabilità del modello di business attuale e a tendere, ma anche valutare la resilienza/stabilità dello stesso e i punti di vulnerabilità e debolezza "critici", che potrebbero mettere a rischio la futura sopravvivenza della banca e che potrebbero non essere messi in luce da altri elementi dello SREP.

Nel framework SREP, la BMA è strutturata nei seguenti passaggi:

- $\quad$ assessment preliminare, che prevede l'inquadramento delle principali attività/aree geografiche e del posizionamento di mercato della banca oggetto di analisi;

- identificazione delle aree di interesse, ossia delle linee di business che maggiormente potrebbero mettere a rischio la solidità del modello di business e la sostenibilità economica futura della banca sotto esame;

- valutazione del contesto imprenditoriale (business environment), anche con riferimento allo scenario macroeconomico e finanziario di mercato e alle strategie attese di "peer group" di riferimento;

- analisi (quantitativa) dell'attuale modello di business, focalizzata sulla verifica della performance economicofinanziaria dell'istituto e della sua coerenza con la propensione al rischio dichiarata; 
- $\quad$ analisi (qualitativa) dell'attuale modello di business, con particolare riferimento ai fattori di successo e di vantaggio competitivo e al valore del franchise;

- $\quad$ analisi quantitativa della strategia forward-looking e dei piani finanziari, per approfondire la plausibilità delle ipotesi manageriali e la rischiosità implicita nella propria strategia di business;

- valutazione della sostenibilità economica (viability) del modello di business e della sostenibilità della strategia, attraverso la quale le autorità di vigilanza competenti sintetizzano il proprio giudizio sul rischio di sostenibilità connesso al modello di business e alla strategia per identificare le cosidette "key vulnerability" e lo scoring finale attribuito alla banca analizzata.

La prassi attraverso la quale le banche supportano l'interlocuzione con i Joint Supervisory Team (JST) nelle analisi di BMA risultano ancora in evoluzione e non pienamente consolidate. Molte delle informazioni che la Vigilanza richiede di acquisire sono infatti disseminate in una pletora di documenti (piani industriali, Tableau de Bord RAF, analisi di posizionamento competitivo, piani di risanamento, etc.) riconducibili a responsabilità diffuse (CRO, Pianificazione Strategica, Pianificazione commerciale, etc.) con un coordinamento interno tra funzioni che viene attivato solo "ad hoc" in occasione della chiamata da parte dei JST. Siamo tuttavia ancora in presenza di una "zona grigia" in cui non c'è certezza su:

- tipologia di informazioni di cui è necessario disporre per dare una risposta efficace alla Vigilanza (manca cioè una sorta di "catalogo" della documentazione BMA);

- esaustività della informativa sviluppata nell'ambito di processi già consolidati (spesso infatti il modello di business non è descritto in modo compiuto in nessun documento aziendale);

- $\quad$ ruoli e responsabilità ricoperti dalle funzioni aziendali con specifico riferimento alla indagine di vigilanza sul modello di business (qual è la figura pivot che si occupa di attivare e coordinare il processo BMA all'interno della banca?).

\section{Come identificare/classificare i business model?}

Nell'ambito del sistema bancario sono stati formulati diversi tentativi di pervenire a una classificazione omogenea dei business model dei principali operatori del settore che supportasse l'analisi delle relazioni causali con le performance e/o con il valore di mercato, e l'analisi industriale dei vari cluster. Questi contributi sono stati prevalentemente basati su logiche/algoritmi di classificazione delle banche alimentati con i (pochi) dati pubblici disponibili, tendenzialmente riconducibili all'informativa di bilancio.

Il razionale sottostante a questi modelli quantitativi di classificazione è che la struttura delle poste di bilancio e fuori bilancio sia in qualche modo rappresentativa della tipologia di business in cui la banca intende competere.

Ad esempio, avendo a disposizione una base dati contenente i dati di bilancio degli istituti oggetto di interesse, si possono utilizzare algoritmi statistici di machine learning per creare cluster di banche omogenee in termini di strutture di bilancio e cercare di derivare, a partire dalle caratteristiche di bilancio, una serie di profili che identificano uno specifico business model. L'approccio basato sui dati di bilancio a livello consolidato presenta alcuni limiti, connessi alla indisponibilità di alcune informazioni qualificanti dai dati finanziari, da un lato, e dalla difficoltà di individuare i "modelli" di business "specializzati" delle varie divisioni/business unit/società strumentali nell'ambito dei gruppi complessi, dall'altro.

Per effetto di questi due fattori l'output di queste analisi spesso consente di individuare un numero limitato di business model e di catalogare i grandi gruppi complessi come "diversificati".

Tuttavia l'analisi a livello di gruppo consente di verificare come il business mix consenta di garantire al meglio la sostenibilità dei risultati nel medio termine, risultando quindi più idonea alla valutazione della solidità complessiva del gruppo.

In una recente analisi condotta da Prometeia sono state testate in particolare due metodologie di clustering (una di tipo deterministico, l'altra di tipo probabilistico) per l'individuazione del business model su un campione di 70 Gruppi bancari di 14 paesi dell'area euro, che coprono oltre il 74\% del totale attivo dell'area euro, rilevati tra il 2016 e il 2017 (cluster analysis condotta sui dati di bilancio consolidato - fonte base dati SNL).

Le due metodologie hanno mostrato una significativa convergenza nei risultati.

A fini di cluster analysis sono state considerate le esposizioni di asset \& liability delle banche del campione relativamente ai seguenti aggregati:

- crediti a clientela;

- crediti Interbancari;

- $\quad$ attività finanziarie in titoli;

- derivati;

- depositi da clientela;

- titoli obbligazionari e altre passività finanziarie;

- debiti interbancari.

Con un algoritmo di clustering deterministico (hard) è stata sviluppata una aggregazione progressiva di gruppi omogenei di banche, in base a:

- distanza euclidea per misurare la somiglianza delle banche in termini di indicatori di bilancio di struttura; 
- $\quad$ metodo di Ward implementato tramite l'algoritmo di Lance-Williams;

- numero di business model identificato tramite test di ipotesi derivanti da 40 diversi test statistici basati sull’omogeneità intra-cluster ed eterogeneità inter-cluster.

Con un algoritmo di clustering probabilistico (fuzzy) si è stimata poi la distribuzione di probabilità di appartenenza di una banca ai tre business model identificati tramite hard clustering:

- Fuzzy c-means algorithm (FCM): minimizzazione della distanza attesa tra osservazioni e centri dei clusters, considerato un determinato livello di incertezza.

Questi esercizi hanno restituito risultati simili, di seguito rappresentati attraverso un indicatore di Z-score:

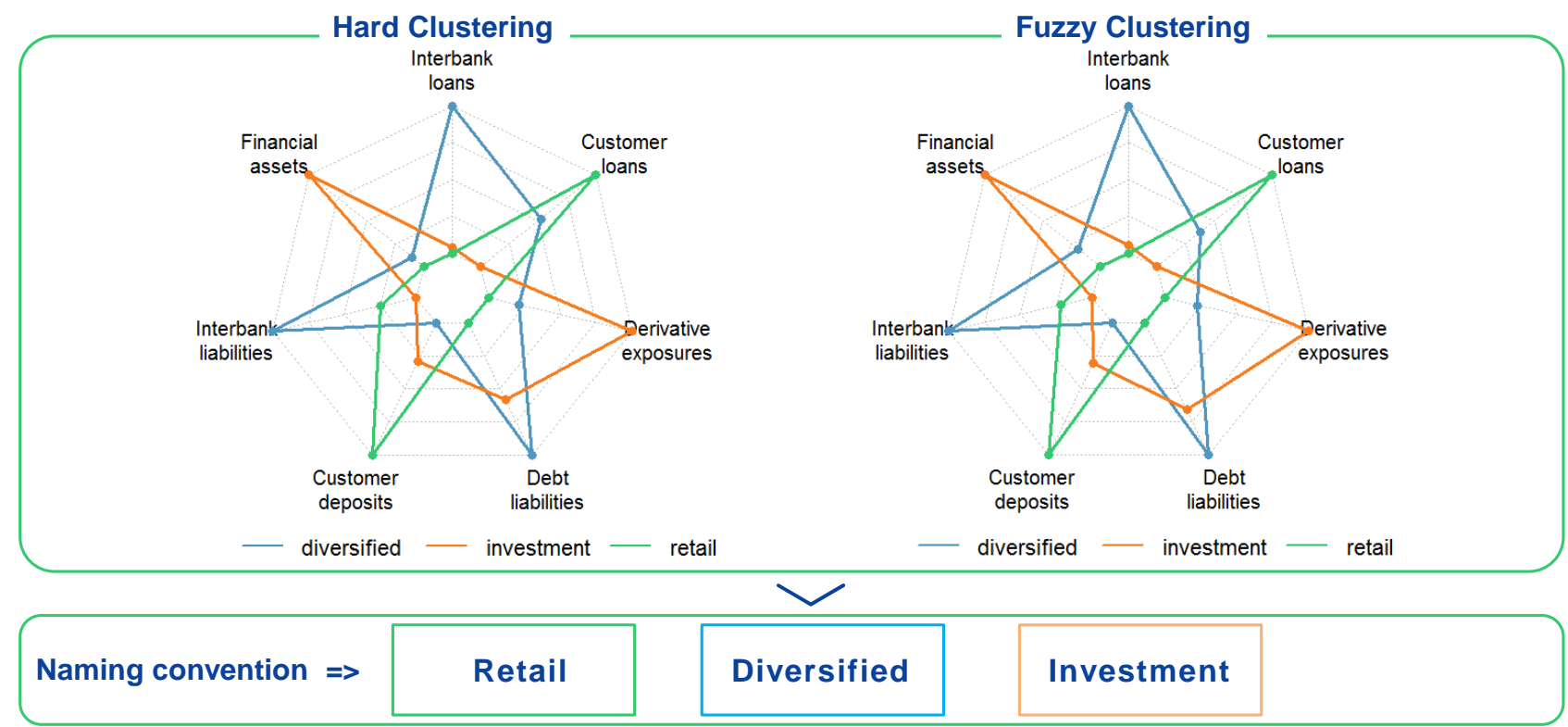

I cluster identificati evidenziano, come da attese, caratteristiche ben differenziate in termini di redditività dell'attivo, costo del rischio ed efficienza (costi/totale attivo) che l'algoritmo ha isolato in tre differenti cluster; attraverso una riconduzione degli "spike" rilevati dall'analisi alle caratteristiche del bilancio si è attribuita ai cluster una label descrittiva, che rappresenta un riferimento indicativo da interpretarsi "per prevalenza". Sono classificate come:

- "retail" le banche che presentano una particolare prevalenza degli aggregati di impieghi e raccolta da clientela;

- "diversified" le banche che presentano una particolare rilevanza delle diverse fonti di funding (interbancario, titoli e passività finanziarie) e di investimenti sull'interbancario attivo;

- “investment" le banche che presentano una particolare prevalenza di esposizioni su attività finanziarie in titoli e derivati. 


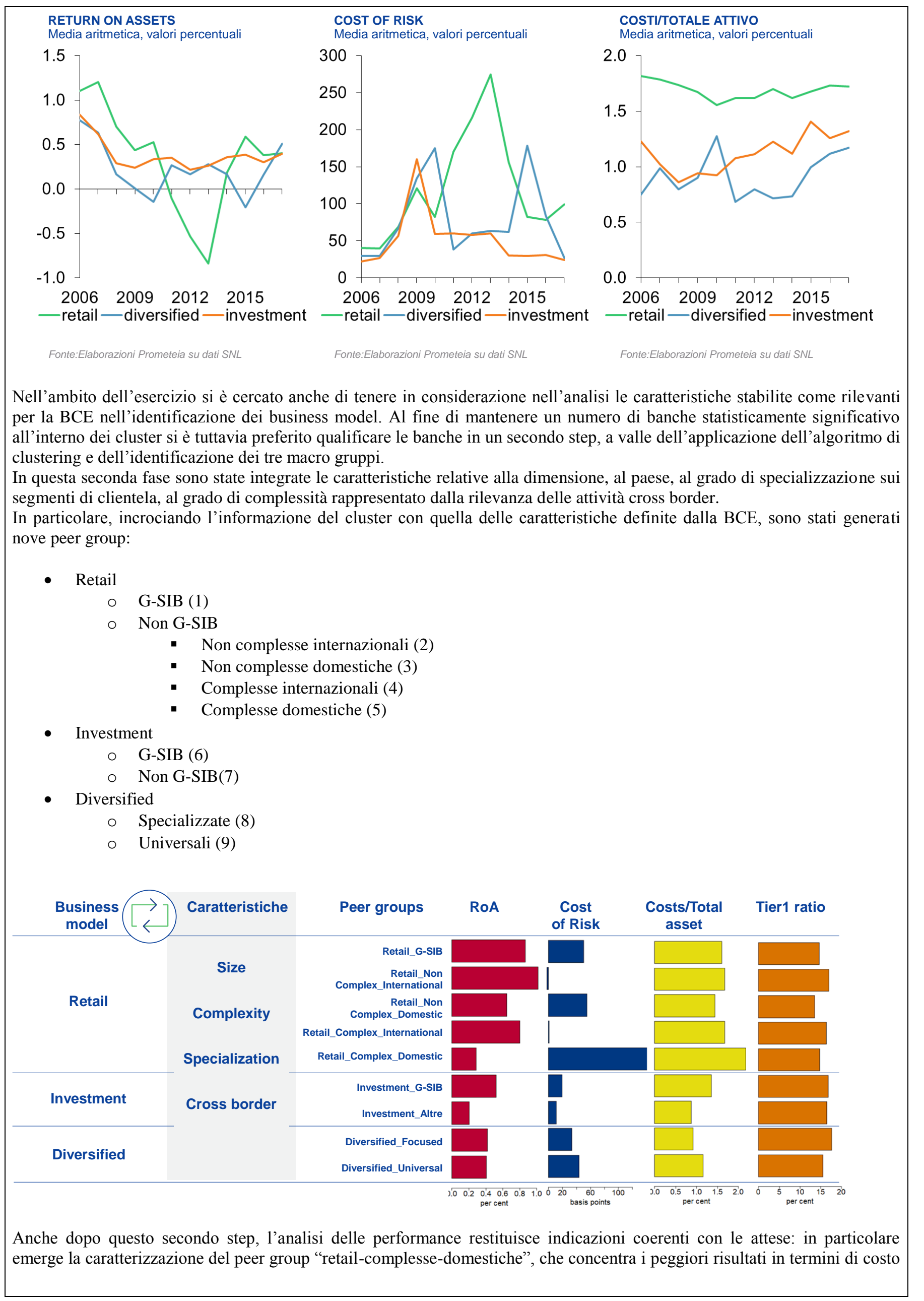


del rischio, mentre le banche internazionali evidenziano risultati più positivi correlati alla capacità di diversificazione del portafoglio.

In parte queste metodologie di lavoro hanno restituito risultati incoraggianti, supportando l'identificazione di business model almeno ad un livello macro di granularità (separando in modo ragionevole le banche commerciali da quelle diversificate e di investimento).

Tuttavia la struttura di bilancio rappresenta solo uno dei potenziali ambiti di interesse per identificare un modello di business. Molti aspetti rilevanti riconducibili agli altri tasselli del modello "canvas" - come partner, risorse chiave, relazioni con i clienti, etc. - non risultano disponibili o sono rappresentati da informazioni qualitative e/o destrutturate che ne rendono difficoltoso l'utilizzo all'interno di una metodologia meramente quantitativa.

Per questo motivo è opportuno integrare i risultati ottenuti dagli algoritmi di classificazione, basati su criteri statistici/quantitativi, con un set di variabili qualitative che contribuiscono a cogliere gli aspetti più immateriali. Queste variabili comprendono:

- $\quad$ il patrimonio di relazioni con gli stakeholder (azionisti, clienti, dipendenti, fornitori, regulator, etc.);

- $\quad$ le strutture societarie e/o di governance, inerenti:

○ i meccanismi decisionali;

- la struttura di governance vera e propria;

- lo stile di management;

- la struttura industriale:

○ i sistemi informativi ed il modello organizzativo;

- la struttura dei vincoli, come:

○ vincoli regolamentari specifici (requisiti regolamentari specifici assegnati/concordati con i regulator, giuslavoristici, etc.);

- vincoli operativi (ad esempio la legacy nelle politiche distributive, clausole di claw back, etc.);

○ vincoli territoriali (ad esempio vincoli di prossimità territoriale)

- etc.

\section{Come cambieranno gli attuali modelli di business?}

Senza dubbio l'innovazione tecnologica (come blockchain, big data, robofinance, AI, machine learning) e la nascita di nuovi operatori (come fintech, challenger bank, open service provider) sono tra i principali fattori di cambiamento che condizioneranno l'evoluzione del modello di business delle banche. Ma in che modo e in quale direzione?

Le nuove tecnologie e i nuovi soggetti consentono di instaurare nuove modalità di relazione con la clientela, nuove modalità operative per gestire e accedere ai prodotti/servizi bancari, nuove opportunità per gestire - e arricchire - il patrimonio informativo tradizionalmente a disposizione delle banche.

Molte banche commerciali sono però cresciute in un mondo in cui non dovevano scegliere quale ruolo ricoprire in relazione all'accesso alla clientela o all'offerta di prodotti. In questo contesto, non era necessaria nessuna differenziazione strategica: molte banche hanno così optato per il posizionamento più universale possibile, con massima ampiezza e profondità di creazione del valore.

In termini strategici, le banche cioè sono spesso rimaste ancorate al loro DNA, continuando a pensare secondo il paradigma implicito nel loro posizionamento di mercato, in base al quale tendono a coprire l'intera catena del valore.

Questo rende impossibile definire e seguire una chiara linea strategica per le attività digitali, con il rischio di sviluppare una collezione non coordinata di iniziative "spot" che tuttavia non possono essere il punto di partenza per il business model digitale del futuro. Le banche devono prendere una decisione consapevole circa il proprio posizionamento strategico, investendoci le giuste energie. Solo allora saranno in grado di competere nella partita del digitale.

Al fine di decidere quale posizione strategica adottare, gli istituti devono prima esaminare criticamente il proprio posizionamento corrente all'interno dell'ecosistema finanziario in evoluzione. La domanda a cui rispondere è: quali sono i punti di forza e quali i punti deboli del modello di business, sia dal punto di vista degli stakeholder esterni (clienti in primis) che da una prospettiva interna?

All'orizzonte vediamo profilarsi un possibile scenario di progressiva specializzazione dei modelli di business verso la gestione di specifici anelli della catena del valore che, conseguentemente, integri a monte o a valle modelli di business di altri operatori (ad esempio attraverso operazioni di M\&A o accordi di partnership con altri operatori specializzati), analogamente a quanto accaduto nel Wealth Management, dove molte banche commerciali hanno scomposto la catena del valore tra produzione, distribuzione, controllo/back office e gestione del post vendita, per concentrarsi sulle attività di distribuzione e gestione del rapporto con il cliente.

In particolare possiamo immaginare tre strategie principali nel prossimo futuro delle banche: relationship leader, originator e technology provider.

\section{Relationship leader}

Anche in un mondo completamente digitale, i clienti continueranno ad avere bisogno di risolvere problemi. I fornitori di servizi finanziari che faranno della risoluzione dei problemi il proprio di forza, potranno posizionarsi con successo nell'interfaccia verso il cliente in qualità di esperti di relazioni. 
Nel ruolo di relationship expert, i fornitori di servizi finanziari si concentreranno sulla comprensione delle esigenze dei clienti e sulla possibilità di offrire un adeguato supporto di consulenza che vada oltre l'offerta di specifici prodotti finanziari e possa estendersi anche ad altri servizi complementari. La chiave del successo di questo posizionamento è il livello di attenzione verso il cliente, e come questo viene percepito dal cliente stesso. Cosa deve accadere perché questo focus sia autentico?

Due aspetti sono decisivi in questo senso:

- al posto della tradizionale mentalità di prodotto orientata al fornitore all'interno della banca, il punto di vista da adottare è la centralità del cliente e la risoluzione dei suoi problemi;

- la disponibilità e la centralità del cliente saranno viste in modo olistico nel contesto di una strategia "omnichannel": il contatto con il cliente, fisico o virtuale, deve avvenire ovunque egli si trovi.

Il business model del relationship expert è caratterizzato dalla remunerazione del servizio di consulenza/brokeraggio con provvigioni e commissioni. La struttura di bilancio di questa tipologia di operatori sarà pertanto capital light e quindi a bassa RWA density in quanto il rischio di credito, parte del rischio di mercato e il rischio di tasso saranno sostenuti dagli operatori che hanno effettivamente iscritto le posizioni dei clienti nei propri libri.

Sarà inoltre knowledge leader nelle metodologie di analisi predittiva e nell'interpretazione (anticipazione) dei bisogni della clientela chi saprà indirizzare la struttura degli investimenti verso l'eccellenza nella gestione delle basi dati e nell'utilizzo di business analytics.

L'esperienza di digitalizzazione di altre industrie indica tuttavia che non tutte le banche saranno in grado di posizionarsi in modo permanente come esperti di relazioni. La concorrenza nell'interfaccia client sarà intensa, non da ultimo a causa della crescente importanza delle piattaforme web, canale attraverso il quale anche player non industriali potranno entrare sul mercato e occupare spazi.

\section{Originator}

Se i punti di forza di una banca risiedono principalmente nell'ottimizzazione dei prodotti e dei processi, essa potrebbe posizionarsi come specialista di prodotto. Questa tipologia di banca, esperta di prodotto, fornisce soluzioni di alta qualità, commercializzate attraverso un'ampia rete di canali di vendita - come le piattaforme - che in futuro tenderanno ad essere gestite da terzi. A differenza delle banche posizionate nell'interfaccia client, l'esperto di prodotto si concentra sul raggiungimento del massimo livello possibile di efficienza nei processi aziendali e sulla realizzazione di una migliore ingegneria di prodotto. Gli esperti mirano a posizionare bene i propri prodotti con i distributori che occupano l'interfaccia del cliente. Il business model degli originator si basa in genere sui tradizionali ricavi da intermediazione (interessi) e/o sui ricavi commissionali, a seconda del prodotto. Gli originator saranno soggetti con forte competenza nella pianificazione finanziaria e nella gestione dei rischi. Saranno con ogni probabilità soggetti eccellenti nei processi di pricing, nelle metodologie e nei processi di allocazione del capitale e nelle attività di capital e liquidity management. Questi soggetti potranno limitare l'utilizzo di canali di vendita diretti (fisici o virtuali) e distribuire i propri prodotti attraverso le piattaforme di mercato e/o accordi distributivi con reti di terze parti.

\section{Technology provider}

Un terzo scenario strategico è quello del technology provider. I fornitori di tecnologia si concentrano sullo sviluppo di soluzioni basate sulla tecnologia per clienti finali o aziende (B2B, B2C) e sulla fornitura di tecnologie in logica di piattaforma. Questo modello ha già acquisito enorme importanza nell'evoluzione delle piattaforme digitali. I processi e i sistemi informatici delle banche spesso non si dimostrano in grado di soddisfare i requisiti digitali; le piattaforme di benchmarking stanno collegando le banche alla loro infrastruttura attraverso l'acquisizione diretta delle banche come utenti (in un modello Platform-as-a-Service). Il modello di business del fornitore di tecnologia è fortemente centrato su commissioni di licenza di accesso e pagamenti di commissioni sull'operatività. Inoltre, tutte le attività e i servizi offerti sono allineati alla tecnologia o all'infrastruttura fornite. Non c'è quindi competizione per l'accesso ai clienti.

Per avere successo come fornitore di tecnologie a lungo termine, sono essenziali un alto livello di competenza tecnologica e la capacità di realizzare economie di scala digitali.

\section{Quali implicazioni industriali}

Il processo di specializzazione dei modelli di business potrebbe portare a una significativa revisione delle strutture di bilancio $\mathrm{e}$, in particolare, delle fonti di profitability nel conto economico delle banche così come le conosciamo ora.

Per quanto riguarda gli "originator" potremmo ad esempio assistere ad una progressiva concentrazione degli asset \& liability del sistema sui bilanci di un numero più limitato di operatori.

Le banche che si focalizzeranno sul modello di relationship leader perderanno una quota di ricavi riconducibile al margine di intermediazione per compensarlo con una quota importante di commissioni sui servizi di advisory e una rilevante diminuzione dei costi delle infrastrutture e del personale impegnati nei processi produttivi. Allo stesso tempo, dovranno gestire un incremento di costi e di investimenti nelle infrastrutture funzionali ad ottimizzare la relazione con la clientela (analytics, modelli predittivi, modelli di advisory, etc.).

Per i technology provider, le piattaforme multi-brand di re-seller o dealer di prodotti e servizi finanziari acquisiranno una quota importante di commissioni per la remunerazione dei servizi di accesso alla clientela finale, anche se dovranno garantire adeguati standard di sicurezza (investimenti in sistemi di cyber security e fraud management) e di qualità nei propri processi 
digitali (investimenti in processi di customer experience). Buona parte della redditività di questi operatori deriverà poi dalla loro effettiva capacità di realizzare economie di scala che transitano dalla gestione accentrata dell'operatività della clientela.

La ricomposizione del settore poterà inoltre a una maggiore contendibilità di specifiche business combination, che potrebbero diventare oggetto di accordi (acquisizioni di start-up tecnologiche, cessioni di piattaforme, consolidamento di centri informatici o unità di back office), funzionali alla definizione di nuovi accordi produttivi e/o distributivi. Questo passaggio favorirà la nascita di operatori specializzati "mono-business" (SPAC, Società di credit recovery, Asset Manager, Servicer amministrativi e/o tecnologici, etc.), contribuendo ulteriormente alla specializzazione dei soggetti nell'ecosistema finanziario.

\section{Bibliografia}

Maurizio Pierigè

- $\quad$ Osterwalder., Y. Pigneur, (2010) creare modelli di business, Milano FAG 2012

- M. Di Antonio, I modelli di business nel settore bancario: un'analisi da ripensare - Bancaria 2018

- $\quad$ S. Cosma, R. Ferretti, E. Gualandri, A. Landi, V. Venturelli: I modelli di business delle banche: letteratura, nuove regole e implicazioni strategiche. S - BANCARIA, 2016

- E. Pelganta, M. Pierigè, L. Prosperi: Le variabili determinanti della redditività e i loro fattori di cambiamento: profilazione delle banche nel contesto (new normal) post crisi; intervento al convegno AIFIRM di Roma 08/08/2018

- $\quad$ E. De Meo, A. De Nicola, G. Lusignani, F. Orsini, L. Zicchino, 2017: European Banks in the 21st Century: are their Business Models profitable?

- Beckert, Hach, Steger: Roland Berger: Third European Retail Banking Survey - Findings and recommendations, august 2018 


\title{
La misurazione dell'esposizione al rischio di tasso di interesse del portafoglio bancario: quali implicazioni in sede ICAAP a seguito della recente introduzione dell'approccio del margine di interesse nel quadro normativo di vigilanza prudenziale?
}

\author{
di $^{1}$ Igor Gianfrancesco ${ }^{2}$ (Extrabanca)
}

\section{Abstract}

According to the new regulatory framework, in their Internal Capital Adequacy Assessment Process (ICAAP) analysis banks should base the contribution of interest rate risk in the banking book (IRRBB) to the overall internal capital by taking into account not only the economic value perspective but also the earning one. The supervisory authorities, however, do not provide detailed information on how to integrate the two approaches. The objective of this paper is twofold. First, it sheds more light on the impact of the new six interest rate shock scenarios recently introduced by the Basel Committee on Banking Supervision to the regulatory framework currently in force to measure banks' exposure to IRRBB. In particular, the study compares the evidence obtained following both the economic value approach and the earning perspective. Secondly, in line with the technical documents produced by the supervisory authorities, it provides a methodological proposal to integrate these results within the ICAAP, based on a solvency indicator that takes into account second pillar risks too. The analysis highlights the importance of declining specific integration criteria in order to calibrate an appropriate amount of internal capital so as to preserve the bank from any adverse interest rates scenarios and, at the same time, not to penalize the credit supply to the economy.

\section{Introduzione}

Il $20^{\circ}$ aggiornamento della Circolare 285/2013 del 21/11/2017 apporta sostanziali modifiche al quadro normativo di vigilanza prudenziale sul rischio di tasso di interesse del portafoglio bancario al fine di recepire le linee guida dell'Autorità Bancaria Europea (ABE) sulla gestione del rischio in questione emanate nel mese di ottobre 2015. Le principali novità introdotte riguardano l'introduzione ai fini della determinazione dell'esposizione al rischio di tasso di interesse del portafoglio bancario dell'approccio del margine di interesse in aggiunta a quello del valore economico, che rappresentava l'unico approccio fino a tale data previsto dalle disposizioni di vigilanza. Le innovazioni regolamentari, inoltre, chiariscono che nella determinazione dell'esposizione al rischio le banche devono utilizzare, sia nell'ambito dell'approccio del valore economico che in quello del margine di interesse, anche scenari diversi di variazione del livello e della forma della curva dei rendimenti. Il disposto normativo richiama, quindi, solo implicitamente i nuovi scenari di variazione dei tassi di interesse introdotti dal Comitato di Basilea nel documento tecnico pubblicato ad aprile 2016 e, successivamente, recepiti dall'ABE nell'aggiornamento delle linee guida emanato in data 19 luglio 2018.

Le modifiche regolamentari si inseriscono in uno scenario finanziario di riferimento caratterizzato da un basso livello dei tassi di interesse che, a seguito dell'applicazione del vincolo di non negatività, ha determinato degli effetti distorsivi dando luogo al fenomeno della neutralità al rischio. Si tratta, in altri termini, di banche non esposte al rischio di tasso di interesse del portafoglio bancario a seguito dell'applicazione delle metodologie di calcolo attualmente in vigore nel quadro regolamentare di vigilanza prudenziale rappresentate dallo scenario parallelo dei $+/-200$ punti base e dal metodo dei percentili ${ }^{3}$. Il rischio in questione, come noto, contribuisce, nell'ambito del processo interno di determinazione dell'adeguatezza patrimoniale (Internal Capital Adequacy Assessment Process - ICAAP), con le altre tipologie di rischio a cui le banche sono esposte alla determinazione del capitale interno complessivo. Le relative evidenze sono, successivamente, analizzate dalle Autorità di Vigilanza in sede di processo di revisione e valutazione prudenziale (Supervisory Review and Evaluation Process - SREP) ai fini della determinazione per ciascuna banca degli Overall Capital Requirement (OCR). Essi sono definiti dalle Guidelines EBA/GL/2014/13 come somma del Total SREP Capital Requirement (TSCR) ratio, dato dalla somma del requisito minimo regolamentare e dal requisito aggiuntivo determinato ad esito dello SREP, e della riserva di conservazione del capitale. Il livello degli OCR ratio, sommato alla eventuale capital guidance disposta dalle stesse Autorità di vigilanza in sede SREP, influenza la crescita dimensionale della banca. L'attività di erogazione del credito è infatti, funzione della differenza tra il profilo di rischio della banca alle varie date di valutazione e i limiti imposti dalle Autorità di vigilanza in sede SREP. Una maggiore esposizione sul rischio di tasso di interesse del portafoglio bancario derivante dalle innovazioni regolamentari oggetto di analisi può, quindi, condurre ad un incremento dei requisiti aggiuntivi in sede SREP, di cui al precedente punto, e, per tale via, ad una potenziale riduzione dell'offerta di credito all'economia. Quanto detto assume, inoltre, particolare

${ }^{1}$ L'articolo è stato inoltre sottoposto a doppio referaggio anonimo, pervenuto in data 22/10/2018 e accettato il 16/11/2018.

${ }^{2}$ Le opinioni espresse sono proprie dell'Autore e non vincolano in alcun modo le Istituzioni di appartenenza

${ }^{3}$ Il fenomeno della neutralità al rischio è stato più volte segnalato nella letteratura di riferimento. Al riguardo si rimanda a Cocozza et. al. (2015), Gianfrancesco (2016 e 2017), Cerrone et al. (2017). 
rilevanza nell'ipotesi di un eventuale futuro aumento del livello della struttura per scadenza dei tassi di interesse. Ciò, infatti, farebbe, progressivamente, venir meno il fenomeno della neutralità al rischio rendendo, quindi, esposte (con un relativo capitale interno da porre a presidio del rischio in questione) tutte quelle banche che attualmente risultano neutrali al rischio.

Il presente contributo prende spunto dalle evidenze fornite in Gianfrancesco (2017), di cui rappresenta un proseguimento, relative all'impatto dei nuovi scenari proposti dal Comitato di Basilea nell'ambito del solo approccio del valore economico e si inserisce in un'area di ricerca che analizza, da differenti prospettive, la robustezza e le relative implicazioni sulla complessiva esposizione al rischio della metodologia di calcolo definita dal Comitato di Basilea nel 2004, e, successivamente, recepita dalla Banca d'Italia prima nella Circolare 263 del dicembre 2010 e poi nella Circolare 285 nel dicembre 2013, per stimare l'esposizione al rischio di tasso di interesse del portafoglio bancario [cfr. Fiori e Iannotti (2007), Entrop et al. (2008), Entrop et al. (2009), Abdymomunov e Gerlach (2014), Cocozza et al. (2015), Cerrone et al. (2017)]. L'AIFIRM ha posto particolare attenzione a tale tematica ospitato vari contributi nella propria Newsletter poi Risk Management Magazine [cfr. Curcio e Gianfrancesco (2012), Gianfrancesco e Giliberto (2014) e Gianfrancesco (2016 e 2017)] oltre a predisporre uno specifico Position Paper nell'ambito della consultazione promossa nel 2015 dal Comitato di Basilea, che ha condotto alla stesura del documento tecnico pubblicato ad aprile 2016. In aggiunta alla precedente letteratura ${ }^{4}$ il presente contributo fornisce una prima stima dell'impatto sull'esposizione al rischio di tasso di interesse del portafoglio bancario dell'applicazione dei sei nuovi scenari definiti dal Comitato di Basilea considerando congiuntamente l'approccio del margine di interesse e del valore economico così come richiesto, di recente, dall'autorità di vigilanza prudenziale. Inoltre, formula specifiche ipotesi metodologiche, in linea con gli orientamenti di vigilanza prudenziale e le prassi di settore, in termini di misurazione del capitale interno complessivo da porre a presidio del rischio in questione mediante l'integrazione delle evidenze ottenute dall'applicazione dei due suddetti approcci.

La trattazione, quindi, si rivolge, in un prospettiva micro, agli operatori del settore al fine di fornire agli stessi utili indicazioni funzionali ad una più appropriata integrazione delle evidenze risultanti dall'applicazione dei due approcci (valore economico e margine di interesse) nella stesura del proprio Resoconto ICAAP e, in generale, ad una più consapevole comprensione delle determinanti dell'esposizione al rischio di tasso di interesse del portafoglio bancario a seguito delle recenti innovazioni regolamentari. Allo stesso tempo, in una prospettiva macro, si propone di fornire agli studiosi della disciplina, posti sulla linea di demarcazione tra ricerca teorica ed applicata, ulteriori stimoli atti a spostare più in avanti l'attuale frontiera dell'indagine scientifica nella comprensione della relazione di interdipendenza tra l'attivo e il passivo di bilancio e delle relative implicazioni in termini di redditività. Il proseguo della trattazione è strutturato come segue: nel paragrafo 2 sono descritte brevemente le metodologie di calcolo nell'ambito dell'approccio del valore economico e del margine di interesse sulla base di quanto disposto dal quadro normativo prudenziale di vigilanza prudenziale. La trattazione prosegue poi fornendo le evidenze relative ad un campione di 130 banche italiane nel periodo che va dal 2006 al 2017. Infine, l'attenzione si focalizza sulle modalità di integrazione tra l'approccio del valore economico e del margine di interesse fornendo, al riguardo, una specifica proposta metodologica basata sul calcolo di un indicatore di solvibilità rettificato che tiene conto non solo dei rischi di I pilastro ma anche di quelli di II pilastro. Il contributo si conclude con le relative implicazioni di policy.

\section{Metodologie}

\subsection{Approccio del valore economico}

La misurazione dell'esposizione al rischio di tasso di interesse del portafoglio bancario mediante l'approccio del valore economico viene effettuata sulla base dell'algoritmo semplificato definito nel Titolo III, Capitolo 1, Allegato C della Circolare 285/2013 emanata dalla Banca d'Italia sulla base delle indicazioni fornite nel documento tecnico di Basilea pubblicato nel $2004^{5}$. Nello specifico, la metodologia proposta: i) prevede la ripartizione dei valori contabili delle attività e delle passività di bilancio sensibili ai tassi di interesse in fasce temporali a cui sono associati specifici coefficienti di duration; ii) prende in considerazione sia una variazione parallela dei tassi di interesse di $+/-200$ punti base, sia il $1^{\circ}$ e il $99^{\circ}$ percentile delle variazioni dei tassi di interesse su base annua registrate in un periodo di osservazione di 6 anni (nel seguito della

\footnotetext{
${ }^{4}$ Per un approfondimento sui vari contributi citati nel presente articolo si rimanda a Gianfrancesco (2017) in cui è riportata una dettagliata rassegna della letteratura sul tema in questione.

${ }^{5}$ L'attenzione verso il rischio di tasso di interesse del portafoglio bancario ha assunto una certa rilevanza a seguito della crisi delle Savings and Loans americane degli anni '80. Subito dopo la crisi, infatti, gli economisti della Federal Reserve System svilupparono il modello Economic Value Model (EVM), adottando per la misurazione di tale tipologia di rischio la prospettiva del valore economico. Il modello è descritto in Houpt e Embersit (1991) e, successivamente, analizzato in Wright e Houpt (1996), Sierra e Yeager (2004) e Sierra (2009). Nel 2004 il Comitato di Basilea ha emanato la versione definitiva del documento tecnico intitolato "Principles for the management of the interest rate risk" contenente 15 principi per la misurazione e la gestione del rischio in questione e una metodologia di misurazione basata sulla stessa logica sottostante il modello sviluppato dalla Federal Reserve System.
} 
trattazione indicati, rispettivamente, con scenario parallelo e metodo dei percentili ${ }^{6}$; iii) calcola un indicatore di rischio dato dal rapporto tra la variazione di valore economico del portafoglio bancario, a seguito della scenario di variazione dei tassi di interesse ipotizzato, e i fondi propri; e, iv) stabilisce, infine, che le Autorità di vigilanza devono prestare particolare attenzione alle c.d. banche "anomale" che presentano un livello dell'indicatore di rischio superiore al $20 \%$. Il procedimento di calcolo sopra descritto deve essere effettuato per ogni valuta rilevante (superiore al $5 \%$ del totale delle attività o passività rientranti nel portafoglio bancario) e per l'aggregato delle valute non rilevanti. Le evidenze ottenute sono, successivamente, sommate tra loro solo se conducono ad una effettiva riduzione di valore economico. Non è, quindi, ammessa la compensazione tra le diverse valute. In simboli, l'indicatore di rischio regolamentare (IR) è calcolato mediante la seguente formula:

$$
\begin{aligned}
& \sum_{j=1}^{k} \sum_{i=1}^{n} P N_{j i} \cdot D M_{j i} \cdot \Delta r_{j i} \\
& I R=\frac{s e>0}{F P}
\end{aligned}
$$

dove, con $\mathrm{j}=1, \ldots \mathrm{k}$ sono indicate le valute rilevanti e l'aggregato delle valute non rilevanti; con $\mathrm{i}=1, \ldots .14$ le fasce temporali della matrice per scadenza e per data di riprezzamento definita dalla normativa di vigilanza prudenziale; $\mathrm{PNi}_{\mathrm{i}} \mathrm{DM}_{\mathrm{i}}$, $\Delta \mathrm{r}_{\mathrm{i}}$ indicano, rispettivamente, la posizione netta, la duration modificata approssimata e la variazione del tasso di interesse associato alla i-esima fascia temporale e, infine, FP il livello dei fondi propri. Per costruzione un valore positivo (negativo) della (1.) corrisponde ad una riduzione (incremento) del valore economico dei fondi propri.

Il documento tecnico del Comitato di Basilea pubblicato nell'aprile del 2016 introduce sei nuovi scenari di variazione dei tassi di interesse da utilizzare ai fini della stima dell'esposizione al rischio di tasso di interesse del portafoglio bancario. Tali scenari sono stati successivamente recepiti dalle linee guida dell'ABE pubblicate lo scorso mese di luglio 2018 . I sei scenari sono di seguito elencati:

i) parallel shock up: scenario parallelo verso l'alto;

ii) parallel shock down: scenario parallelo verso il basso;

iii) short rates shock up: spostamento verso l'alto di tutti i tassi di interesse con maggior ampiezza su quelli a breve termine;

iv) short rates shock down: spostamento verso il basso di tutti i tassi di interesse con maggiore ampiezza su quelli a breve termine;

v) steepener shock: riduzione dei tassi di interesse a breve e medio termine e incremento di quelli a lungo termine;

vi) flattener shock: incremento dei tassi di interesse a breve e medio termine e riduzione di quelli a lungo termine.

La successiva Tabella 1 riporta i vari scenari di variazione dei tassi di interesse considerando anche l'applicazione del vincolo di non negatività, con riferimento ai dati al 31/12/2017, disposto dall'attuale quadro normativo di vigilanza prudenziale.

Lo scenario parallelo verso l'alto e verso il basso coincide in termini di ampiezza con quello attualmente in vigore di +/-200 punti base ${ }^{7}$. Gli scenari di variazione dei tassi di interesse short rates shock up e short rates shock down sono tra loro simmetrici e caratterizzati da variazioni dello stesso segno, rispettivamente, positive e negative, che diminuiscono via via in termini di ampiezza in corrispondenza delle varie fasce temporali da quella "a vista" fino a quella "oltre i 20 anni". Nell'ultima fascia temporale si ha per entrambi una variazione pari a zero. Gli scenari di variazione dei tassi di interesse steepener e flattener sono, invece, caratterizzati da variazioni di differente segno sulle varie fasce temporali della matrice per scadenza e per data di revisione. Il primo è caratterizzato da variazioni negative via via decrescenti in ampiezza dalla fascia temporale "a vista "fino a quella "da 3 anni a 4 anni" e da variazioni positive via via crescenti in ampiezza dalla fascia "da 4 a 5 anni" fino a quella "oltre i 20 anni”. Il secondo, invece, da variazioni positive via via decrescenti in ampiezza dalla fascia "a

${ }^{6}$ In linea con quanto disposto dal Comitato di Basilea nel documento tecnico del 2004 e dalla Banca d'Italia nella Circolare 285/2013 nell'ambito del metodo dei percentili si utilizza ai fini del calcolo delle variazioni su base annua la tecnica delle osservazioni sovrapposte. In base a tale tecnica le variazioni su base annua dei tassi di interesse sono calcolate come differenza tra i livelli osservati in un dato anno e quelli rilevati nell'anno precedente. Utilizzando per ciascun nodo della struttura a termine dei tassi di interesse una serie storica di sei anni di livelli su base giornaliera precedenti la data di valutazione si ottiene una serie storica di cinque anni di variazioni su base annua.Nello specifico il metodo dei percentili prevede la determinazione, in corrispondenza di ciascuna fascia temporale, del $1^{\circ}$ e del $99^{\circ}$ percentile della distribuzione storica delle variazioni su base annua del relativo tasso di interesse. In tal modo si ottengono due differenti scenari. Il primo scenario composto per ciascuna fascia temporale da variazioni, in genere, negative dei tassi di interesse; il secondo caratterizzato, invece, per ciascuna fascia temporale, da variazioni, in genere, positive.

${ }^{7}$ Ciò si verifica per specifiche valute di denominazione tra cui l'euro come si può osservare dalle relative modalità di determinazione degli scenari per un approfondimento delle quali si riporta allo stesso documento tecnico pubblicato dal Comitato di Basilea o alle recenti linee guida dell'ABE. 
vista" fino a quella "da 4 a 5 anni" e da variazioni negative via via crescenti in ampiezza dalla fascia "da 5 a 7 anni”" a "oltre i 20 anni".

Tabella 1. Scenari di variazione dei tassi di interesse (Valori percentuali)

\begin{tabular}{|c|c|c|c|c|c|c|c|c|c|c|c|c|c|c|}
\hline \multirow{2}{*}{$\begin{array}{c}\text { Fasce } \\
\text { temporali }\end{array}$} & \multirow{2}{*}{$\begin{array}{c}\text { Livello } \\
\text { tassi di } \\
\text { interesse }\end{array}$} & \multicolumn{3}{|c|}{ Scenario parallelo } & \multicolumn{3}{|c|}{$\begin{array}{l}\text { Metodo dei } \\
\text { percentili }\end{array}$} & \multicolumn{3}{|c|}{$\begin{array}{c}\text { Short rates } \\
\text { up/down }\end{array}$} & \multicolumn{4}{|c|}{ Steepener/Flattener } \\
\hline & & 200 & -200 & $\mathbf{V N}$ & $99^{\circ}$ & $1^{\circ}$ & VN & SRU & SRD & $\mathbf{V N}$ & STEEP & $\mathbf{V N}$ & FLAT & VN \\
\hline A vista & $-0,35$ & 2,00 & $-2,00$ & 0,00 & 0,27 & $-1,09$ & 0,00 & 2,50 & $-2,50$ & 0,00 & $-1,63$ & 0,00 & 2,00 & 2,00 \\
\hline Fino a 1m & $-0,37$ & 2,00 & $-2,00$ & 0,00 & 0,15 & $-1,27$ & 0,00 & 2,47 & $-2,47$ & 0,00 & $-1,60$ & 0,00 & 1,97 & 1,97 \\
\hline $1 \mathrm{~m}-3 \mathrm{~m}$ & $-0,33$ & 2,00 & $-2,00$ & 0,00 & 0,14 & $-1,28$ & 0,00 & 2,40 & $-2,40$ & 0,00 & $-1,52$ & 0,00 & 1,89 & 1,89 \\
\hline $3 m-6 m$ & $-0,27$ & 2,00 & $-2,00$ & 0,00 & 0,22 & $-1,37$ & 0,00 & 2,28 & $-2,28$ & 0,00 & $-1,40$ & 0,00 & 1,77 & 1,77 \\
\hline $6 \mathrm{~m}-1^{\circ}$ & $-0,19$ & 2,00 & $-2,00$ & 0,00 & 0,25 & $-1,42$ & 0,00 & 2,07 & $-2,07$ & 0,00 & $-1,19$ & 0,00 & 1,56 & 1,56 \\
\hline $1 \mathrm{a}-2^{\circ}$ & $-0,15$ & 2,00 & $-2,00$ & 0,00 & 0,15 & $-1,40$ & 0,00 & 1,72 & $-1,72$ & 0,00 & $-0,84$ & 0,00 & 1,19 & 1,19 \\
\hline $2 a-3^{\circ}$ & 0,01 & 2,00 & $-2,00$ & $-0,01$ & 0,27 & $-1,57$ & $-0,01$ & 1,34 & $-1,34$ & $-0,01$ & $-0,45$ & $-0,01$ & 0,79 & 0,79 \\
\hline $3 a-4^{\circ}$ & 0,16 & 2,00 & $-2,00$ & $-0,16$ & 0,37 & $-1,63$ & $-0,16$ & 1,04 & $-1,04$ & $-0,16$ & $-0,15$ & $-0,15$ & 0,48 & 0,48 \\
\hline $4 a-5^{\circ}$ & 0,30 & 2,00 & $-2,00$ & $-0,30$ & 0,42 & $-1,63$ & $-0,30$ & 0,81 & $-0,81$ & $-0,30$ & 0,08 & 0,08 & 0,24 & 0,24 \\
\hline $5 a-7^{\circ}$ & 0,56 & 2,00 & $-2,00$ & $-0,56$ & 0,47 & $-1,58$ & $-0,56$ & 0,56 & $-0,56$ & $-0,56$ & 0,34 & 0,34 & $-0,02$ & $-0,02$ \\
\hline $7 a-10^{\circ}$ & 0,89 & 2,00 & $-2,00$ & $-0,89$ & 0,50 & $-1,51$ & $-0,89$ & 0,30 & $-0,30$ & $-0,30$ & 0,60 & 0,60 & $-0,29$ & $-0,29$ \\
\hline $10 a-15 a$ & 1,26 & 2,00 & $-2,00$ & $-1,26$ & 0,50 & $-1,59$ & $-1,26$ & 0,11 & $-0,11$ & $-0,11$ & 0,79 & 0,79 & $-0,49$ & $-0,49$ \\
\hline $15 a-20 a$ & 1,43 & 2,00 & $-2,00$ & $-1,43$ & 0,51 & $-1,66$ & $-1,43$ & 0,03 & $-0,03$ & $-0,03$ & 0,87 & 0,87 & $-0,57$ & $-0,57$ \\
\hline Oltre 20a & 1,49 & 2,00 & $-2,00$ & $-1,49$ & 0,52 & $-1,66$ & $-1,49$ & 0,00 & 0,00 & 0,00 & 0,90 & 0,90 & $-0,59$ & $-0,59$ \\
\hline
\end{tabular}

Note: $200=$ scenario parallelo dei +200 punti base; $-200=$ scenario parallelo dei -200 punti base; $99^{\circ}=$ scenario del $99^{\circ}$ percentile; $1^{\circ}=$ scenario del $1^{\circ}$ percentile; SRU = scenario dello short rates shock up; SRD = scenario dello short rates shock down; STEEP = scenario steepener; FLAT = scenario flattener. VN indica l'applicazione del vincolo di non negatività sulla base del livello dei tassi di interesse al 31/12/2017 allo scenario indicato nella Tabella alla colonna precedente.

Fonte: Elaborazioni dell'Autore su dati Datastream.

Le variazioni dei tassi di interesse al ribasso devono essere corrette al fine di rispettare il vincolo di non negatività. Ai fini della corretta applicazione di tale vincolo occorre distinguere a seconda che il livello vigente dei tassi di interesse alla data di valutazione sia maggiore o minore di zero. Nel dettaglio, possono verificarsi le seguenti casistiche a seconda che il livello del tasso di interesse vigente alla data di valutazione in corrispondenza di una specifica fascia temporale è:

i) positivo e superiore o uguale, in valore assoluto, alla variazione negativa da applicare: si applica la variazione negativa propria dello scenario oggetto di applicazione;

ii) positivo e inferiore, in valore assoluto, alla variazione negativa da applicare: si applica una variazione negativa di uguale ampiezza al livello del tasso di interesse vigente alla data di valutazione, in modo che quest'ultimo a seguito dell'applicazione dello scenario utilizzato per la stima dell'esposizione al rischio assuma valori non inferiori a zero;

iii) negativo: si applica in base alle recenti indicazioni fornite dall'Autorità Bancaria Europea in una recente Frequently Asked Questions (FAQ) del luglio 2017 (Question ID 2017_3121) una variazione pari a zero. Dalla $F A Q$ si deduce, infatti, che il floor dello $0 \%$ si applica solo in corrispondenza di scenari di variazione dei tassi negativi sui nodi della struttura per scadenza dei tassi di interesse dove si registrano livelli positivi. In precedenza alla predetta $F A Q$ la prassi di settore considerava, in genere, una variazione positiva di pari ampiezza al livello negativo del tasso di interesse vigente alla data di valutazione tale da ricondurre quest'ultimo ad un livello pari zero.

È interessante notare nella Tabella 1 che dalle fasce temporali da "a vista" fino a quella "da 1 anno a 2 anni" il livello vigente dei tassi di interesse è negativo. In tal caso in corrispondenza dell'adozione di scenari caratterizzati da variazioni negative l'applicazione del vincolo di non negatività impone di considerare una variazione pari a zero così come specificato nel precedente punto iii). Nelle fasce temporali successive "da 2 anni a 3 anni" fino a quella "oltre i 20 anni”" si registrano, invece, valori positivi ma comunque contenuti. In tali casi, se la variazione negativa ipotizzata è maggiore, in valore assoluto, del livello vigente alla data di valutazione l'applicazione del vincolo di non negatività richiede di considerare una variazione negativa esattamente pari al livello del tasso di interesse in modo tale da non condurre lo stesso ad assumere valori inferiori a zero. Quanto appena descritto è stato fatto, ad esempio, per lo scenario parallelo dei -200 punti e per quello del $1^{\circ}$ percentile. Inoltre, dato il basso livello dei tassi di interesse al 31/12/2017 e la conseguente applicazione del vincolo di non negatività, i due predetti scenari sono caratterizzati per ogni fascia temporale da variazioni della stessa ampiezza. Se, invece, il livello positivo del tasso di interesse è maggiore, in valore assoluto, delle variazioni negative ad essi associate si utilizzano le stesse variazione negative cosi come fatto, ad esempio, per il SRD dalle fasce temporali da "5 anni a 7 anni" fino a quella "da 15 anni a 20 anni”. Analoghe considerazioni valgono per lo scenario del flattener, che non è caratterizzato dall'applicazione del 
vincolo di non negatività in quanto le variazioni negative di cui alle fasce temporali "da 5 a 7 anni "fino a quella "oltre i 20 anni" sono minori in valore assoluto del livello dei relativi livelli dei tassi di interesse vigenti alla data di valutazione.

\subsection{Approccio del margine di interesse}

Il $20^{\circ}$ aggiornamento della Circolare 285 del 21/11/2017, al paragrafo 2.2. della Parte Prima, Titolo III, Capitolo 1, Sezione III) relativo al calcolo del capitale interno in condizioni ordinarie introduce ai fini della stima della complessiva esposizione al rischio di tasso di interesse del portafoglio bancario l'approccio del margine di interesse, in aggiunta a quello già previsto del valore economico. Nello specifico, il dettato normativo stabilisce che per determinare l'esposizione al rischio di tasso di interesse del portafoglio bancario, le banche misurano le potenziali variazioni sia del valore economico sia del margine di interesse o degli utili attesi, considerando scenari diversi di variazione del livello e della forma della curva dei rendimenti.

La misurazione dell'esposizione al rischio di tasso di interesse del portafoglio bancario nella prospettiva reddituale avviene, nella prassi operativa, su un orizzonte temporale di breve periodo (detto gapping period) posto, di solito, pari ad 1 anno. Nella presente trattazione si utilizza il modello del repricing gap che calcola la variazione attesa del margine di interesse a seguito di una variazione attesa dei tassi di interesse. La metodologia richiede, in corrispondenza di ogni fascia temporale, di calcolare il relativo gap periodale, che coincide con le posizioni nette nell'ambito dell'approccio del valore economico. In altre parole, le varie poste di bilancio sono classificate in funzione dei criteri definiti dal quadro normativo di vigilanza prudenziale sul rischio di tasso di interesse del portafoglio bancario. Il gap periodale è, successivamente, moltiplicato per la variazione di tasso ipotizzata e per il periodo di tempo in cui tale variazione ha effetto. In particolare, si ipotizza che tutte le scadenze e le revisioni di tasso all'interno di una specifica fascia temporale avvengono nel punto medio della stessa. In termini formali la variazione attesa del margine di interesse a seguito di una variazione attesa dei tassi di interesse risulta pari $a^{8}$ :

$$
E(\Delta M I)=\sum_{i=1}^{n} G A P_{i} \cdot\left(T-t^{*}\right) \cdot E\left(\Delta r_{i}\right)
$$

dove:

- $\mathrm{GAP}_{\mathrm{i}}$ indica il gap periodale relativo all'i-esimo fascia temporale;

- T-t* indica l'intervallo di tempo intercorrente tra il punto medio della fascia temporale e la fine del gapping period;

- $t^{*}=\frac{t_{i}+t_{i-1}}{2}$ è il punto medio della fascia temporale che ha per estremi i punti $\mathrm{t}_{\mathrm{i}} \mathrm{e} \mathrm{t}_{\mathrm{i}-1}$;

- $\Delta \mathrm{r}_{\mathrm{i}}$ indica la variazione del tasso di interesse associato nell'i-esima fascia temporale.

In base alla (2.), quindi, il gap periodale, o in altri termini la posizione netta, è la variabile che lega la variazione attesa del margine di interesse allo shock di tasso di interesse ipotizzato. Inoltre, una data variazione dei tassi di interessi esercita i propri effetti ell'intervallo di tempo intercorrente tra il punto medio della relativa fascia temporale e la fine del gapping period. Per costruzione, un valore negativo (positivo) della (2.) corrisponde ad una riduzione (incremento) del margine di interesse.

${ }^{8}$ Il modello di seguito descritto si basa sulle seguenti ipotesi semplificatrici:

i. le attività e le passività sensibili prese in considerazione sono solo quelle esistenti alla data di valutazione; vengono, quindi, trascurate le variazioni relative ai volumi intermediati, che possono verificarsi all'interno dell'orizzonte temporale di riferimento;

ii. ogni attività o passività sensibile in scadenza viene rinnovata automaticamente con un'operazione di natura analoga, dello stesso ammontare e di durata pari o superiore alla rimanente durata del gapping period. Gli interessi maturati sia sulle poste sensibili sia su quelle insensibili non sono presi in considerazione nel modello;

iii. si analizzano gli effetti di una sola variazione dei tassi di mercato all'interno del gapping period;

iv. si ipotizza che la variazione dei tassi di mercato avviene un istante immediatamente successivo la data di classificazione delle poste di bilancio in sensibili e non sensibili e ha effetti sulle attività e passività sensibili per tutto il periodo di riferimento. La variazione dei tassi di mercato, inoltre, impatta allo stesso modo (con la stessa intensità) su tutte le attività sensibili e le passività onerose. In altre parole, si esclude l'ipotesi che i tassi applicati su alcune poste di bilancio si adeguino in maniera più o meno marcata rispetto alle altre alle variazioni dei tassi di mercato;

v. si utilizza, inoltre, nel calcolo il regime della capitalizzazione semplice. 


\section{Le implicazioni sull'esposizione complessiva al rischio}

\subsection{Dati descrittivi}

Il presente paragrafo riporta le evidenze relative all'impatto dei differenti scenari di variazione dei tassi di interesse descritti nel precedente paragrafo 2 su un campione di 130 banche commerciali italiane nel periodo 2006-2017. L'analisi è effettuata considerando i dati di bilancio pubblicati al 31/12 di ogni anno. Nello specifico i dati utilizzati sono quelli riportati nella matrice per scadenza e data di riprezzamento pubblicata nel bilancio di esercizio di fine anno nella Parte E della nota integrativa intitolata "Informazioni sui rischi e sulle relative politiche di copertura". Per i dati relativi alla dotazione patrimoniale si è fatto riferimento, invece, alla parte E della nota integrativa intitolata "Informazioni sul patrimonio" Il campione è costituito da banche, principalmente di piccola e media dimensione rientranti nella classe 3 ai fini ICAAP, per le quali sono disponibili i dati nell'intero periodo di riferimento'.

La seguente tabella 2 riporta la composizione dei principali aggregati dell'attivo e del passivo di bilancio nell'intero periodo oggetto di analisi, in percentuale del totale attività e passività per cassa comprensive del valore nominale delle posizioni fuori bilancio ${ }^{10}$. Le quote percentuali sono calcolate per ogni anno come media semplice dei valori ottenuti sulle singole banche. L'analisi dei dati evidenzia, nel corso del periodo oggetto di analisi, dal lato dell'attivo un incremento del peso dei titoli, che è passato dal 14,46\% nel 2006 al 28,62\% nel 2017. Si registra, allo stesso tempo, una contestuale riduzione dei prestiti erogati dal 76,18\% al 62,08\%. Dal lato del passivo si segnala, invece, dal un lato un incremento della componente a vista da clientela al dettaglio dal $38,50 \%$ al $51,13 \%$ e della componente altra raccolta a medio termine (da 1 anno a 5 anni), al cui interno rientra parte della raccolta verso la BCE, dall' $1,51 \%$ al $11,61 \%$ e, dall'altro lato, una riduzione dei titoli obbligazionari emessi dal $28,55 \%$ al $13,40 \%$.

Tabella 2. Composizione attivo e passivo di bilancio del campione di banche (Valori percentuali)

\begin{tabular}{|c|c|c|c|c|c|c|c|c|c|c|c|c|}
\hline & 2006 & 2007 & 2008 & 2009 & 2010 & 2011 & 2012 & 2013 & 2014 & 2015 & 2016 & 2017 \\
\hline Titoli fasce temp. inf. a 1a & 10,00 & 9,47 & 11,78 & 12,20 & 11,80 & 11,23 & 12,41 & 14,81 & 19,08 & 18,92 & 18,51 & 17,60 \\
\hline Titoli fasce temp. 1a e 5a & 3,16 & 2,56 & 1,23 & 2,05 & 2,06 & 2,95 & 6,48 & 7,53 & 6,73 & 6,57 & 6,74 & 6,99 \\
\hline Titoli fasce temp. sup. ai 5a & 1,30 & 1,15 & 0,68 & 1,39 & 1,73 & 1,94 & 3,72 & 5,27 & 5,08 & 5,93 & 5,99 & 4,03 \\
\hline Prestiti fasce temp. inf. a 1a & 19,52 & 23,90 & 24,49 & 25,98 & 29,65 & 26,90 & 22,65 & 20,63 & 20,62 & 21,74 & 22,49 & 23,73 \\
\hline Prestiti fasce temp. 1a e 5a & 5,77 & 3,71 & 4,01 & 5,68 & 5,00 & 6,56 & 5,77 & 6,39 & 6,02 & 7,12 & 7,24 & 7,52 \\
\hline Prestiti fasce temp. sup. ai $5 \mathrm{a}$ & 5,17 & 3,37 & 4,56 & 5,61 & 3,31 & 4,32 & 2,87 & 2,72 & 2,82 & 3,11 & 3,85 & 4,85 \\
\hline Pos. lunghe fuori bilancio & 9,37 & 8,83 & 7,39 & 6,73 & 8,98 & 9,66 & 8,64 & 8,16 & 7,86 & 6,81 & 6,94 & 7,94 \\
\hline CC passivi da clientela & 38,50 & 37,45 & 37,18 & 41,00 & 40,04 & 37,42 & 33,80 & 35,09 & 37,87 & 43,28 & 47,59 & 51,13 \\
\hline Altre componenti a vista & 9,24 & 9,22 & 9,12 & 9,18 & 9,88 & 9,30 & 10,19 & 8,38 & 8,12 & 8,02 & 7,78 & 8,66 \\
\hline Raccolta fasce temp. inf. a 1a & 12,13 & 9,76 & 7,97 & 4,85 & 5,69 & 8,88 & 15,77 & 18,40 & 17,83 & 16,07 & 12,38 & 7,01 \\
\hline Raccolta fasce temp. 1a e 5a & 1,45 & 0,93 & 0,44 & 0,27 & 0,37 & 1,55 & 1,57 & 2,18 & 2,42 & 4,05 & 7,75 & 11,61 \\
\hline Raccolta fasce temp. sup. ai 5 a & 0,33 & 0,19 & 0,20 & 0,19 & 0,19 & 0,18 & 0,16 & 0,17 & 0,23 & 0,29 & 0,31 & 0,28 \\
\hline Titoli fasce temp. inf. a 1a & 20,74 & 25,03 & 29,09 & 28,17 & 21,27 & 19,27 & 15,36 & 13,93 & 12,10 & 10,10 & 8,37 & 6,89 \\
\hline Titoli fasce temp. tra 1 a e $5 a$ & 7,09 & 7,58 & 7,99 & 8,76 & 12,53 & 12,74 & 13,56 & 12,55 & 11,66 & 10,34 & 8,10 & 6,08 \\
\hline Titoli fasce temp. ai $5 a$ & 0,72 & 0,57 & 0,36 & 0,41 & 0,65 & 0,72 & 0,65 & 0,72 & 0,83 & 0,77 & 0,55 & 0,43 \\
\hline Pos. corte fuori bilancio & 38,50 & 37,45 & 37,18 & 41,00 & 40,04 & 37,42 & 33,80 & 35,09 & 37,87 & 43,28 & 47,59 & 51,13 \\
\hline
\end{tabular}

Fonte: elaborazioni dell'Autore sui dati di bilancio al 31/12 di ogni anno.

Le analisi empiriche effettuate nel proseguo della trattazione fanno riferimento all'attuale matrice per scadenza e per data di riprezzamento utilizzata ai fini della stima dell'indicatore di rischio regolamentare e composta da 14 fasce temporali ${ }^{11}$.

${ }^{9}$ La Circolare 262 del 22 dicembre 2015 che disciplina gli schemi e le regole di compilazione del bilancio bancario stabilisce che la tabella concernente la distribuzione delle poste di bilancio per data di scadenza e di riprezzamento può non essere redatta se nella nota integrativa viene fornita un'analisi di sensitività del rischio di tasso di interesse in base ai modelli interni o ad altre metodologie che soddisfi quanto richiesto in materia di IFRS7. Quando detto fa sì che nella costituzione del campione sono state escluse quelle banche che non hanno pubblicato la tabella in questione anche in un solo anno del periodo oggetto di analisi. Le banche che scelgono di inserire nella nota integrativa le proprie analisi di sensitività invece della matrice per scadenza e per data di riprezzamento sono, da quanto emerso nell'ambito della fase di raccolta di dati, in genere quelle di maggiori dimensioni. Le banche escluse sono, quindi, quelle per le quali non sono disponibili i dati utilizzati nella presente analisi nel periodo 2006-2017. Il campione è da ritenersi rappresentativo delle banche commerciali italiane di piccole e medie dimensioni. I controlli effettuati sulle altre banche per gli anni in cui si rendono disponibili i dati confermano, in generale, le evidenze ottenute nel presente contributo. La scelta di un ampio periodo di riferimento consente di ottenere evidenze empiriche concernenti sia la dinamica dei tassi di interesse sia le modifiche intervenute sulla composizione delle poste di bilancio. Il 2006 rappresenta, inoltre, il primo anno in cui sono disponibili gli schemi di bilancio utilizzati nell'analisi.

${ }^{10} \mathrm{Si}$ precisa che la collocazione temporale delle varie poste di bilancio a scadenza nella Tabella 1 fa riferimento ai criteri di classificazione nelle varie fasce temporali definiti dal quadro normativo di vigilanza prudenziale nella prospettiva del rischio di tasso di interesse del portafoglio bancario. Di conseguenza gli aggregati a tasso variabile sono ricondotti alla fascia temporale coincidente la prima data di revisione del tasso di interesse.

${ }^{11}$ La matrice per scadenza e per data di revisione riportata nei bilanci bancari presenta, tuttavia, un numero di fasce temporali inferiori a quelle di cui alla matrice regolamentare. I dati sono stati, quindi, redistribuiti nelle varie fasce temporali della matrice regolamentare in proporzione del numero di mesi contenenti nelle stesse. Ad esempio, i volumi contenuti nella fascia temporale da 1 mese a 3 mesi della matrice di bilancio sono stati redistribuiti per $1 / 3$ nella fascia temporale fino ad 1 mese e per il $2 / 3$ nella fascia temporale da oltre 1 mese 
Per l'intero periodo di riferimento si utilizza il criterio di distribuzione dei depositi introdotto nel 2011 che prevede la ripartizione di tali poste di bilancio nelle fasce temporali fino a $5 \mathrm{anni}^{12}$. Ai fini della stima dell'indicatore di rischio si utilizza, inoltre, una struttura per scadenza di 14 tassi di interesse (detti in genere anche key-rates) i cui nodi corrispondono alla scadenza a vista e ai punti medi delle altre 13 fasce temporali della matrice per scadenza e per data di riprezzamento fornita dalle Autorità di vigilanza. I key-rates utilizzati, in linea con le attuali prassi di settore, sono il tasso EONIA per la scadenza a vista, i tassi Euribor per le scadenze inferiori a 12 mesi e i tassi Swap per le scadenze uguali o superiori a 1 anno ${ }^{13}$. La successiva Tabella 3 riporta per l'intero periodo di riferimento 2006-2017 il peso delle posizioni nette sui fondi propri, in corrispondenza delle varie fasce temporali della matrice per scadenza e data di riprezzamento, relativo all'intero campione oggetto di analisi calcolati per ogni anno come media semplice dei valori ottenuti sulle singole banche.

Tabella 3. Struttura per scadenza delle posizioni nette (Valori percentuali)

\begin{tabular}{|c|c|c|c|c|c|c|c|c|c|c|c|c|c|c|}
\hline Anno & $\begin{array}{c}\text { A } \\
\text { vista }\end{array}$ & $\begin{array}{c}\text { Fino a } \\
1 \mathrm{~m}\end{array}$ & $\begin{array}{c}1 \mathrm{~m}- \\
3 \mathrm{~m}\end{array}$ & $\begin{array}{l}3 \mathrm{~m}- \\
6 \mathrm{~m}\end{array}$ & $\begin{array}{c}6 \mathrm{~m}- \\
1 \mathrm{a}\end{array}$ & $\begin{array}{l}1 \mathrm{a}- \\
2 \mathbf{a}\end{array}$ & $\begin{array}{c}2 a- \\
3 a\end{array}$ & $\begin{array}{l}3 a- \\
4 a\end{array}$ & $\begin{array}{l}4 a- \\
5 a\end{array}$ & $\begin{array}{c}5 a- \\
7 a\end{array}$ & $\begin{array}{l}\text { 7a- } \\
10 a\end{array}$ & $\begin{array}{l}10 a- \\
15 a\end{array}$ & $\begin{array}{l}15 a- \\
20 a\end{array}$ & $\begin{array}{c}\text { Oltre } \\
20 a\end{array}$ \\
\hline 2006 & 2,18 & $-0,10$ & $-0,21$ & $-0,46$ & $-0,32$ & $-0,39$ & $-0,39$ & $-0,39$ & $-0,39$ & 0,12 & 0,17 & 0,07 & 0,07 & 0,07 \\
\hline 2007 & 2,44 & $-0,02$ & $-0,05$ & $-0,42$ & $-0,13$ & $-0,43$ & $-0,43$ & $-0,43$ & $-0,43$ & 0,08 & 0,12 & 0,05 & 0,05 & 0,05 \\
\hline 2008 & 2,59 & 0,03 & 0,06 & $-0,44$ & $-0,27$ & $-0,51$ & $-0,51$ & $-0,51$ & $-0,51$ & 0,08 & 0,11 & 0,08 & 0,08 & 0,08 \\
\hline 2009 & 2,12 & 0,17 & 0,34 & $-0,34$ & $-0,28$ & $-0,54$ & $-0,54$ & $-0,54$ & $-0,54$ & 0,13 & 0,19 & 0,09 & 0,09 & 0,09 \\
\hline 2010 & 2,03 & 0,36 & 0,72 & $-0,18$ & $-0,30$ & $-0,63$ & $-0,63$ & $-0,63$ & $-0,63$ & 0,09 & 0,13 & 0,08 & 0,08 & 0,08 \\
\hline 2011 & 2,30 & 0,12 & 0,23 & $-0,32$ & $-0,20$ & $-0,60$ & $-0,60$ & $-0,60$ & $-0,60$ & 0,16 & 0,25 & 0,10 & 0,10 & 0,10 \\
\hline 2012 & 2,25 & $-0,01$ & $-0,01$ & 0,00 & $-0,37$ & $-0,50$ & $-0,50$ & $-0,50$ & $-0,50$ & 0,20 & 0,29 & 0,09 & 0,09 & 0,09 \\
\hline 2013 & 2,10 & $-0,12$ & $-0,24$ & 0,42 & $-0,42$ & $-0,52$ & $-0,52$ & $-0,52$ & $-0,52$ & 0,24 & 0,35 & 0,10 & 0,10 & 0,10 \\
\hline 2014 & 1,65 & $-0,06$ & $-0,11$ & 1,02 & $-0,36$ & $-0,61$ & $-0,61$ & $-0,61$ & $-0,61$ & 0,27 & 0,41 & 0,09 & 0,09 & 0,09 \\
\hline 2015 & 1,22 & $-0,18$ & $-0,37$ & 1,26 & $-0,46$ & $-0,70$ & $-0,70$ & $-0,70$ & $-0,70$ & 0,27 & 0,40 & 0,10 & 0,10 & 0,10 \\
\hline 2016 & 0,80 & 0,31 & 0,63 & 1,16 & $-0,50$ & $-0,85$ & $-0,85$ & $-0,85$ & $-0,85$ & 0,32 & 0,48 & 0,10 & 0,10 & 0,10 \\
\hline 2017 & 0,51 & 0,59 & 1,18 & 1,09 & $-0,44$ & $-0,95$ & $-0,95$ & $-0,95$ & $-0,95$ & 0,31 & 0,47 & 0,14 & 0,14 & 0,14 \\
\hline Media & 1,85 & 0,09 & 0,18 & 0,23 & $-0,34$ & $-0,60$ & $-0,60$ & $-0,60$ & $-0,60$ & 0,19 & 0,28 & 0,09 & 0,09 & 0,09 \\
\hline
\end{tabular}

Fonte: Elaborazioni dell'Autore su dati di bilancio al 31/12 di ogni anno.

È interessante osservare che i criteri di allocazione definiti dal quadro normativo di vigilanza prudenziale ${ }^{14}$ conducono, in genere, ad una struttura per scadenza delle posizione nette caratterizzata da posizioni nette negative nelle fasce a medio termine da 1 anno a 5 anni riconducibili, essenzialmente, al peso dei depositi a vista che, come noto, sono classificati in un orizzonte temporale di 5 anni e alle altre forme tecniche di raccolta a scadenza come i depositi vincolati e le obbligazioni emesse che hanno, in genere, scadenza nel medio termine e da posizioni nette positive nella fasce temporali a lungo termine oltre i 5 anni riconducibili alla scadenza delle quote capitali dei mutui a tasso fisso e dei titoli a tasso fisso iscritti nel portafoglio bancario in scadenza su tale orizzonte temporale. Le posizioni nette negative a seguito di un aumento (diminuzione) dei key-rates danno luogo a posizioni nette ponderate negative (positive) e quindi ad un incremento (riduzione) del valore economico dei fondi propri. Al contrario le posizioni nette positive a seguito di un aumento (diminuzione) dei keyrates danno luogo a posizione nette ponderate positive (negative) e quindi ad una riduzione (incremento) del valore economico dei fondi propri. Le posizioni nette ponderate positive e negative che si formano, invece, sulle fasce temporali a breve termine entro un anno non impattano in maniera significativa sulla stima dell'indicatore di rischio dati $\mathrm{i}$ bassi coefficienti di duration ad esse associati. In tali fasce temporali rientrano le poste di bilancio a tasso variabile, quelle a tasso fisso in scadenza entro l'anno e la parte dei depositi a vista distribuita in tale orizzonte temporale. La variazione di valore economico dei fondi propri, che rappresenta il numeratore dell'indicatore di rischio regolamentare, dipende quindi: i) dall'entità e dal segno delle posizioni nette in corrispondenza di ogni fascia temporale; ii) dalla duration ad esse associate; e iii) dall'ampiezza e dal segno delle variazioni dei key-rates a seconda dello specifico scenario utilizzato. L'ammontare dei fondi propri influenza il calcolo dell'indicatore di rischio in quanto computato al denominatore dello stesso e, quindi, rileva ai fini della verifica del mantenimento di un'esposizione al rischio sotto la soglia di allerta fissata dalle Autorità di vigilanza.

fino a 3 mesi e così via. Nell'ambito della presente analisi è stata utilizzata una unica matrice per scadenza e per data di revisione relativa alla valuta di denominazione euro. Se non disponibile si è fatto riferimento alla matrice per scadenza e per data di riprezzamento relativa all'intero portafoglio bancario denominato in euro. In entrambi i casi le modalità di calcolo dell'indicatore di rischio regolamentare di cui alla formula (1.) e la variazione del margine di cui alla formula (2.) sono stati applicati per una unica valuta rilevante di denominazione.

${ }^{12} \mathrm{~L}$ 'attuale quadro normativo di vigilanza prudenziale dispone che i depositi a vista sono allocati per il $25 \%$ nella fascia temporale a vista e la rimanente parte nelle fasce temporali che coprono un orizzonte temporale di 5 anni in proporzione al numero di mesi in esse contenuti.

${ }^{13}$ In corrispondenza dei punti medi delle fasce temporali della matrice per scadenza e per data di riprezzamento per i quali non è disponibile un tasso di interesse di mercato il relativo key-rate è ottenuto, in genere nella prassi gestionale e nelle analisi riportate nel presente contributo, considerando quello associato all'estremo superiore della specifica fascia temporale.

${ }^{14}$ Per un maggior dettaglio sui criteri di allocazione delle varie poste di bilancio nella fasce temporali della matrice regolamentare si rimanda alla Circolare 272/2008 e successivi aggiornamenti emanata dalla Banca d'Italia. 
La successiva Tabella 4 riporta i livelli dei tassi di interesse al 31/12 nei vari anni oggetto di analisi. Le evidenze mostrano che la struttura per scadenza dei key-rates ha subito nel corso degli anni una tendenziale riduzione dei livelli in corrispondenza di tutti i nodi, ed in particolare su quelli a breve termine, che ha condotto ad una maggiore inclinazione della stessa. La progressiva riduzione dei livelli ha condotto all'applicazione del vincolo di non negatività sugli scenari di variazione in diminuzione dei tassi di interesse richiesti dal quadro normativo di vigilanza prudenziale. Ad esempio a partire dal 2009 si registrano sulle fasce temporali che vanno da quella a vista fino a quella da oltre 1 anno a 2 anni livelli inferiori ai 200 punti base che hanno condotto all'applicazione del vincolo di non negatività sullo scenario parallelo dei -200 punti base. A partire dallo stesso anno il vincolo di non negatività è stato applicato anche al metodo dei percentili, allo short rates shock down e allo steepener. Inoltre, negli ultimi due anni si registrano valori negativi dei key-rates sui nodi della curva a breve e medio termine. Ciò fa sì che in base all'applicazione del vincolo di non negatività su tali nodi l'utilizzo di scenari di variazione su base annua dei tassi di interesse negativi conduce a considerare variazioni pari a zero, così come specificato nelle recenti indicazioni fornite dall'Autorità Bancaria Europea riportate nel precedente paragrafo 2.

Tabella 4. Struttura per scadenza dei key-rates associati alle varie fasce temporali della matrice per scadenza e data di revisione al 31/12 di ogni anno

(Valori percentuali)

\begin{tabular}{|c|c|c|c|c|c|c|c|c|c|c|c|c|c|c|}
\hline \multirow[b]{2}{*}{$\begin{array}{c}\text { Data di } \\
\text { valutazione }\end{array}$} & \multicolumn{14}{|c|}{ Fasce temporali } \\
\hline & $\begin{array}{c}\text { A } \\
\text { vista }\end{array}$ & $\begin{array}{l}\text { Fino } \\
\text { a } 1 \mathrm{~m}\end{array}$ & $\begin{array}{c}1 \mathrm{~m}- \\
3 \mathrm{~m}\end{array}$ & $\begin{array}{l}3 \mathrm{~m}- \\
6 \mathrm{~m}\end{array}$ & $\begin{array}{c}6 \mathrm{~m}- \\
1 \mathrm{a}\end{array}$ & $1 a-2 a$ & $2 a-3 a$ & $3 a-4 a$ & $4 a-5 a$ & $5 a-7 a$ & $\begin{array}{l}7 \mathbf{a}- \\
10 a\end{array}$ & $\begin{array}{c}10 a- \\
15 a\end{array}$ & $\begin{array}{c}15 a- \\
20 a\end{array}$ & $\begin{array}{c}\text { Oltre } \\
20 a\end{array}$ \\
\hline $31 / 12 / 2006$ & 3,69 & 3,63 & 3,66 & 3,85 & 3,95 & 4,13 & 4,13 & 4,13 & 4,13 & 4,15 & 4,20 & 4,27 & 4,31 & 4,31 \\
\hline $31 / 12 / 2007$ & 3,92 & 4,29 & 4,49 & 4,71 & 4,73 & 4,55 & 4,53 & 4,53 & 4,56 & 4,61 & 4,72 & 4,86 & 4,91 & 4,91 \\
\hline $31 / 12 / 2008$ & 2,35 & 2,60 & 2,79 & 2,97 & 3,02 & 2,76 & 2,96 & 3,12 & 3,24 & 3,46 & 3,74 & 3,90 & 3,86 & 3,67 \\
\hline $31 / 12 / 2009$ & 0,41 & 0,45 & 0,56 & 0,99 & 1,13 & 1,86 & 2,26 & 2,56 & 2,81 & 3,21 & 3,60 & 3,96 & 4,07 & 4,02 \\
\hline $31 / 12 / 2010$ & 0,82 & 0,78 & 0,89 & 1,23 & 1,37 & 1,56 & 1,95 & 2,21 & 2,48 & 2,89 & 3,31 & 3,64 & 3,70 & 3,67 \\
\hline $31 / 12 / 2011$ & 0,63 & 1,02 & 1,18 & 1,62 & 1,79 & 1,32 & 1,38 & 1,53 & 1,73 & 2,07 & 2,37 & 2,67 & 2,69 & 2,62 \\
\hline $31 / 12 / 2012$ & 0,13 & 0,11 & 0,15 & 0,32 & 0,43 & 0,37 & 0,46 & 0,61 & 0,77 & 1,13 & 1,57 & 2,02 & 2,17 & 2,22 \\
\hline $31 / 12 / 2013$ & 0,45 & 0,22 & 0,26 & 0,39 & 0,48 & 0,54 & 0,77 & 1,00 & 1,26 & 1,68 & 2,16 & 2,59 & 2,71 & 2,74 \\
\hline $31 / 12 / 2014$ & 0,14 & 0,02 & 0,04 & 0,17 & 0,25 & 0,18 & 0,22 & 0,29 & 0,36 & 0,53 & 0,82 & 1,15 & 1,33 & 1,42 \\
\hline $31 / 12 / 2015$ & $-0,13$ & $-0,21$ & $-0,17$ & $-0,04$ & 0,00 & $-0,03$ & 0,06 & 0,19 & 0,33 & 0,62 & 1,00 & 1,40 & 1,57 & 1,60 \\
\hline $31 / 12 / 2016$ & $-0,33$ & $-0,37$ & $-0,34$ & $-0,22$ & $-0,14$ & $-0,16$ & $-0,10$ & $-0,03$ & 0,08 & 0,31 & 0,66 & 1,03 & 1,18 & 1,22 \\
\hline $31 / 12 / 2017$ & $-0,35$ & $-0,37$ & $-0,33$ & $-0,27$ & $-0,19$ & $-0,15$ & 0,01 & 0,16 & 0,3 & 0,56 & 0,89 & 1,26 & 1,43 & 1,49 \\
\hline
\end{tabular}

Fonte: Elaborazioni dell'Autore su dati Datastream.

\subsection{Le evidenze empiriche relative all'approccio del valore economico}

La seguente Tabella 5 riporta nel corso del periodo oggetto di analisi il numero di banche esposte nell'ambito dell'approccio del valore economico ai vari scenari di variazione dei tassi di interesse considerati nella presente analisi e rappresentati da quelli attualmente in vigore e da quelli proposti dal Comitato di Basilea nel 2016 e recepiti a luglio 2017 dalle linee guida EBA. I vari scenari sono stati considerati in maniera congiunta a due a due in quanto caratterizzati da un certo grado di simmetria in termini di segni e/o variazioni sulle varie fasce temporali della matrice per scadenza e per data di riprezzamento. Come detto in precedenza per la valuta di denominazione euro, a cui si fa riferimento nella presente analisi, vi è coincidenza tra lo scenario parallelo considerato nel quadro normativo attuale e quello proposto dal Comitato di Basilea. Gli scenari di variazione dei tassi di interesse applicati ai fini della stima dell'esposizione al rischio sono di seguito riportati:

a) shock parallelo verso l'alto e verso il basso di +/- 200 punti base (parallel shock up e down);

b) $1^{\circ}$ e $99^{\circ}$ percentile delle variazioni su base annua dei tassi di interesse degli ultimi 6 anni (metodo dei percentili);

c) shock verso l'alto (basso) sul breve termine (short rates shock up e short rates shock down);

d) shock verso il basso (alto) sul breve termine e verso l'alto (basso) sul lungo termine (steepener e flattener).

In corrispondenza dei suddetti scenari congiunti di variazione dei tassi di interesse sono, inoltre, riportati i casi di neutralità al rischio (banche non esposte ai due scenari considerati in maniera congiunta) e di doppia esposizione (banche esposta a entrambi i due scenari considerati in maniera congiunta).

Lo scenario parallelo dei $+/-200$ punti base è caratterizzato da variazioni della stessa ampiezza e segno opposto su tutte le fasce temporali in assenza di applicazione del vincolo di non negatività. Una banca risulta, in genere, esposta allo scenario in aumento dei tassi di interesse se la riduzione di valore economico associate alle posizioni nette positive è maggiore in valore assoluto dell'incremento di valore economico associato alle posizioni nette negative. Nel caso di una banca esposta a variazioni in aumento dei tassi di interesse la riduzione di valore economico si registra, in genere, sulle fasce temporali a lungo termine dove sono presenti posizioni nette positive riconducibili alla scadenza delle quote capitali dei mutui a tasso fisso e dei titoli a tasso fisso iscritti nel portafoglio bancario. 
Tabella 5. Numero di banche esposte in corrispondenza dei vari scenari di variazione dei tassi di interesse (approccio del valore economico)

\begin{tabular}{|c|c|c|c|c|c|c|c|c|c|c|c|c|c|c|c|c|}
\hline & $(+200)$ & $(-200)$ & $\mathbf{N}$ & D & $99^{\circ}$ & $1^{\circ}$ & $\mathbf{N}$ & D & SRU & SRD & $\mathbf{N}$ & D & STEEP & FLAT & $\mathbf{N}$ & D \\
\hline 2006 & 43 & 87 & 0 & 0 & 35 & 95 & 0 & 0 & 10 & 120 & 0 & 0 & 127 & 1 & 2 & 0 \\
\hline 2007 & 29 & 101 & 0 & 0 & 22 & 107 & 1 & 0 & 6 & 124 & 0 & 0 & 124 & 2 & 2 & 2 \\
\hline 2008 & 24 & 106 & 0 & 0 & 16 & 106 & 8 & 0 & 3 & 127 & 0 & 0 & 129 & 0 & 1 & 0 \\
\hline 2009 & 35 & 91 & 4 & 0 & 21 & 107 & 1 & 1 & 8 & 119 & 2 & 1 & 130 & 0 & 0 & 0 \\
\hline 2010 & 23 & 106 & 1 & 0 & 12 & 117 & 0 & 1 & 2 & 127 & 1 & 0 & 128 & 0 & 2 & 0 \\
\hline 2011 & 41 & 79 & 10 & 0 & 32 & 96 & 2 & 0 & 8 & 121 & 0 & 1 & 129 & 0 & 1 & 0 \\
\hline 2012 & 60 & 20 & 50 & 0 & 37 & 21 & 72 & 0 & 12 & 107 & 10 & 1 & 129 & 0 & 0 & 1 \\
\hline 2013 & 72 & 23 & 35 & 0 & 43 & 29 & 58 & 0 & 13 & 113 & 4 & 0 & 128 & 0 & 0 & 2 \\
\hline 2014 & 68 & 16 & 46 & 0 & 42 & 16 & 72 & 0 & 13 & 75 & 41 & 1 & 125 & 0 & 1 & 4 \\
\hline 2015 & 67 & 6 & 57 & 0 & 36 & 6 & 88 & 0 & 9 & 54 & 65 & 2 & 126 & 0 & 0 & 4 \\
\hline 2016 & 57 & 2 & 71 & 0 & 87 & 2 & 41 & 0 & 5 & 7 & 118 & 0 & 125 & 0 & 4 & 1 \\
\hline 2017 & 57 & 3 & 70 & 0 & 101 & 3 & 26 & 0 & 6 & 54 & 70 & 0 & 129 & 0 & 0 & 1 \\
\hline Media & 48,00 & 53,33 & 28,67 & $\mathbf{0 , 0 0}$ & 40,33 & 58,75 & 30,75 & $\mathbf{0 , 1 7}$ & 7,92 & 95,67 & 25,92 & $\mathbf{0 , 5 0}$ & 127,42 & 0,25 & 1,08 & 1,25 \\
\hline
\end{tabular}

Note: $200=$ scenario parallelo dei +200 punti base; $-200=$ scenario parallelo dei -200 punti base; $99^{\circ}=$ scenario del $99^{\circ}$ percentile; $1^{\circ}=$ scenario del $1^{\circ}$ percentile; SRU = scenario dello short rates shock up; SRD = scenario dello short rates shock down; STEEP = scenario steepener; FLAT $=$ scenario flattener . $\mathrm{N}$ e D indicano, rispettivamente, il numero di banche neutrali al rischio e esposte ad entrambi gli scenari in corrispondenza di ogni scenario congiunto. Fonte: Elaborazioni dell'Autore su dati di bilancio al 31/12 di ogni anno e su dati Datastream.

Nel caso, invece, di banche esposte a variazioni in diminuzione dei tassi di interesse la riduzione di valore economico si registra, invece, sulle fasce temporali a medio termine dove sono presenti posizioni nette negative riconducibili, principalmente, al peso dei depositi a vista e alle altre forme tecniche di raccolta a tasso fisso che scadono in tali fasce temporali.

Data la funzione di trasformazione delle scadenze, basata sul finanziamento di attività a lungo termine con passività a breve e medio termine, una banca dovrebbe risultare esposta a variazioni in aumento dei tassi di interesse. La riduzione del valore economico associata alle attività a seguito di un aumento dei tassi di interesse dovrebbe, infatti, risultare maggiore dell'incremento registrato sulle passività comportando, di conseguenza, una complessiva riduzione del valore economico dei fondi propri. Nella realtà operativa, tuttavia, data l'applicazione dei criteri di allocazione alle varie poste di bilancio, che ne modificano l'originaria durata contrattuale si rileva la presenza, nel periodo oggetto di analisi, non solo di banche esposte a variazioni in aumento dei tassi di interesse, ma anche di banche esposte a variazioni in diminuzione dei tassi di interesse. Le evidenze empiriche della precedente Tabella 5 mostrano, inoltre, una maggiore presenza di banche esposte a variazioni in diminuzione dei tassi di interesse (53,33 in termini di valori medi sull'intero periodo) rispetto a quelle esposte a variazioni in aumento dei tassi di interesse $(48,00)$. Ciò è, principalmente, riconducibile ad un maggior peso nel portafoglio crediti di erogazioni a tasso variabile allocate per l'intero debito residuo nella fascia temporale corrispondente alla prima data di rinegoziazione del tasso e ad un elevato ammontare di depositi a vista allocati sull'orizzonte temporale dei cinque anni.

Tuttavia, come già sottolineato dalla letteratura di riferimento [cfr. Cocozza et al (2015), Gianfrancesco (2016), Cerrone et al. (2017) e Gianfrancesco (2017)], l'applicazione dello scenario parallelo dei +/-200 punti base può dare luogo al fenomeno della neutralità al rischio. Si tratta, nello specifico, di banche per le quali si rileva un incremento del valore economico dei fondi propri in corrispondenza di entrambi gli scenari di variazione dei tassi di interesse considerati in maniera congiunta. L'applicazione del vincolo di non negatività dei tassi di interesse è alla base del fenomeno della neutralità al rischio per lo scenario parallelo dei +/-200 punti base. Nel dettaglio, nell'ipotesi di utilizzo dello scenario parallelo di -200 punti base non corretto per tener conto del vincolo di non negatività la banca sarebbe stata esposta a variazioni in diminuzione dei tassi di interesse, facendo registrare una riduzione del valore economico dei fondi propri di ammontare, in valore assoluto, esattamente pari all'incremento registrato a seguito dell'applicazione dello scenario parallelo di +200 punti base. L'imposizione del vincolo di non negatività nel caso di adozione dello scenario parallelo dei -200 punti base smorza, di fatto, la riduzione di valore economico associata alle posizioni nette negative registrata nelle fasce a medio termine rendendo la banca neutrale al rischio.

L'applicazione del vincolo di non negatività, infatti, in caso di bassi livelli dei tassi di interesse ma positivi riduce l'ampiezza delle variazioni in diminuzione e, quindi, conduce ad un minore incremento del valore economico dei fondi propri, oppure, nel caso di livelli sotto lo zero impone una variazione pari a zero. Le banche neutrali al rischio sono, quindi, banche che in assenza dell'applicazione del vincolo di non negatività sarebbero esposte a variazioni in diminuzione dei tassi di interesse. Le evidenze empiriche riportate nella precedente Tabella 3 mostrano un numero medio di banche neutrali al rischio nel corso del periodo oggetto di analisi pari a 28,67. Nei primi tre anni dal 2006 al 2009 non si registrano fenomeni di neutralità dato l'elevato livello dei tassi di interesse che, come detto, non ha comportato per lo scenario parallelo dei -200 punti base l'attivazione del vincolo di non negatività. Negli anni successivi si registrano, invece, fenomeni di neutralità al 
rischio data, come detto, l'applicazione del vincolo di non negatività con valori più elevati negli ultimi due anni 2016 e 2017 pari, rispettivamente, a 71 e 70 .

Considerazioni sostanzialmente analoghe in termini di determinanti dell'esposizione al rischio valgono per il metodo dei percentili con la differenza che gli scenari di variazione dei tassi associati al $1^{\circ}$ e al $99^{\circ}$ percentile non sono simmetrici cosi come accade invece per lo scenario parallelo dei +/-200 punti base. In tal caso l'applicazione del vincolo di non negatività non è una condizione necessaria affinché si verifichi il fenomeno della neutralità al rischio. In differenti date di valutazione, la dinamica dei tassi di interesse osservata nei 6 anni precedenti può, infatti, condurre, come effettivamente riscontrato nelle evidenze empiriche relative agli anni 2007 e 2008, a particolari scenari associati al $1^{\circ}$ e al $99^{\circ}$ percentile delle distribuzioni delle variazioni su base annua dei key-rates, che, combinati con la struttura per scadenza delle posizioni nette di una banca, generano il fenomeno della neutralità al rischio a prescindere dall'attivazione o meno del vincolo di non negatività. Anche per il metodo dei percentili, così come per lo scenario parallelo dei +/-200 punti base, si rileva un maggiore numero di banche esposte a variazioni in diminuzione dei tassi di interesse rispetto a quelle esposte a variazioni in aumento in termini di valori medi sull'intero periodo oggetto di analisi $(58,75$ vs 40,33$)$. Il numero delle banche neutrali al rischio risulta in media pari a 30,75 con valori decisamente più elevati a partire dal 2012. In corrispondenza dell'adozione di tali scenari si registrano anche due casi di doppia esposizione al rischio negli anni 2009 e 2010 che conducono ad un valore medio sull'intero periodo pari a 0,17 .

Gli scenari di variazione dei tassi di interessi basati sullo shock a breve termine (short rates shock up e short rates shock down) sono caratterizzati da variazioni dello stesso segno (positive e negative) dei tassi di interesse che diminuiscono via via di ampiezza a partire dalla fascia temporale "a vista" fino a quella "Oltre i 20 anni". In corrispondenza dell'ultima fascia temporale si ha una variazione pari a zero. La particolare struttura per scadenza delle posizioni nette delle banche italiane fa sì che le stesse non siano particolarmente esposte allo scenario di variazione verso l'alto. Ciò in quanto le variazioni di maggiore ampiezza sulle fasce a medio termine dove si registrano posizioni nette negative conducono, in genere, ad un incremento di valore economico dei fondi propri maggiore in valore assoluto del decremento associato alle fasce temporali a lungo termine dove si registrano, invece, posizioni nette positive. Al contrario, le banche italiane sono, in genere, esposte allo scenario di variazioni verso il basso. Ciò in quanto le variazioni di maggiore ampiezza sulle fasce a medio termine dove si registrano posizioni nette negative conducono, in genere, ad una riduzione di valore economico dei fondi propri maggiore in valore assoluto dell'incremento associato alle fasce temporali a lungo termine dove si registrano, invece, posizioni nette positive.

Le evidenze empiriche confermano quanto sopra esposto: in termini di valori medi sull'intero periodo oggetto di analisi si registrano un numero di banche esposte allo scenario in aumento e in diminuzione pari, rispettivamente, a 7,92 e 95,67. Le evidenze empiriche mostrano, infine, nel corso degli ultimi anni una riduzione delle banche esposte allo scenario in diminuzione per via dell'applicazione del vincolo di non negatività, che ha smorzato di fatto l'effetto sopra descritto sulle fasce temporali a breve termine generando il fenomeno della neutralità al rischio. Il numero delle banche neutrali si attesta, in media sull'intero periodo, a 25,92, con un significativo incremento negli ultimi tre anni. Dati gli scenari in aumento e in diminuzione caratterizzati, senza l'applicazione del vincolo di non negatività, da variazioni di segno opposto ma della stessa ampiezza valgono considerazioni analoghe a quelle fatte per lo scenario parallelo dei +/-200 punti base in merito al legame tra neutralità al rischio e applicazione del vincolo di non negatività. Inoltre, si rilevano nel corso del periodo alcuni casi di doppia esposizione per un valore medio di 0,50

Lo steepener e il flattener sono scenari di variazione dei tassi di interesse caratterizzati da differenti ampiezze e segni sulle varie fasce temporali della matrice per scadenza e per data di riprezzamento. Nello specifico, lo steepener è caratterizzato da variazioni negative via via decrescenti in ampiezza dalla fascia temporale "a vista "fino a quella "da 3 anni a 4 anni" e da variazioni positive via via crescenti in ampiezza dalla fascia "da 4 a 5 anni" fino a quella "oltre i 20 anni". Il flattener è, invece, caratterizzato da variazioni positive via via decrescenti in ampiezza dalla fascia "a vista" fino a quella "da 4 a 5 anni" e da variazioni negative via via crescenti in ampiezza dalla fascia "da 5 a 7 anni" a quella "oltre i 20 anni”. La particolare struttura per scadenza delle posizioni nette delle banche italiane fa sì che le stesse non siano particolarmente esposte al flattener. Ciò in quanto le variazioni positive sulle fasce a medio termine e negative su quelle a lungo termine conducono ad un incremento del valore economico dei fondi propri date le relative posizioni nette, rispettivamente, negative e positive ad esse associate. Di conseguenza l'applicazione di tale scenario conduce, in genere, su tutte le fasce temporali a medio e lungo termine della matrice per scadenza e per data di riprezzamento ad un incremento del valore economico dei fondi propri. Al contrario, le banche italiane, invece, risultano, in genere, esposte allo steepener in quanto la particolare struttura per scadenza delle posizioni nette fa sì che si registri una riduzione di valore economico sia sulle fasce a medio termine, data l'applicazione di variazioni negative a posizioni nette negative (ad eccezione della fascia temporale "da 4 a 5 anni”), sia su quelle a lungo termine, data l'applicazione di variazioni positive a posizioni nette positive. 
Quanto detto trova conferma nelle evidenze empiriche del periodo oggetto di analisi: in termini di valori medi si registra un numero di banche esposte allo steepener ed al flattener pari, rispettivamente, a 127,42 e 0,25. Non si registrano, inoltre, elevati fenomeni di neutralità al rischio: in termini di valori medi sull'intero periodo le banche neutrali si attestano a 1,08. L'applicazione del vincolo di non negatività, così come per il metodo dei percentili, non è una condizione necessaria affinché si verifichi il fenomeno della neutralità al rischio. Al riguardo è interessante osservare che il flattener è caratterizzato da variazioni negative solo sulle fasce a lungo termine più basse in valore assoluto del relativo livello dei key-rates osservato nel corso degli anni. Tale scenario non è stato, quindi, oggetto di applicazione del vincolo di non negatività. Lo steepener è caratterizzato, invece, da variazioni negative sulle fasce a breve e medio termine. L'applicazione del vincolo di non negatività in contesti di bassi livelli dei tassi di interesse riduce (nel caso di livelli dei tassi vigenti alla data di valutazione positivi) o annulla (nel caso di livelli dei tassi vigenti alla data di valutazione negativi) la corrispondente riduzione di valore economico attenuando, di fatto, la complessiva esposizione al rischio. Quest'ultima è, quindi, riconducibile, principalmente, alle fasce a lungo termine dove si registra una riduzione di valore economico a seguito dell'applicazione di variazioni positive dei tassi di interesse a posizioni nette positive. Inoltre, si rilevano nel corso del periodo alcuni casi di doppia esposizione per un valore medio di 1,25 .

La seguente Tabella 6 riporta il livello medio dell'indicatore di rischio calcolato sulle sole banche esposte in corrispondenza dei singoli scenari di variazione dei tassi di interesse.

Tabella 6. Livello medio dell'indicatore di rischio delle banche esposte in corrispondenza dei vari scenari di variazione dei tassi di interesse (approccio del valore economico)

(Valori percentuali)

\begin{tabular}{|c|c|c|c|c|c|c|c|c|}
\hline Anno & $(+200)$ & $(-200)$ & $99^{\circ}$ & $1^{\circ}$ & SRU & SRD & STEEP & FLAT \\
\hline 2006 & 14,72 & 8,86 & 6,85 & 8,94 & 2,36 & 5,32 & 4,41 & 0,37 \\
\hline 2007 & 11,00 & 7,89 & 4,74 & 8,47 & 2,27 & 5,43 & 3,70 & 1,74 \\
\hline 2008 & 11,69 & 7,71 & 4,72 & 5,15 & 2,12 & 6,36 & 4,40 & 0,00 \\
\hline 2009 & 13,48 & 7,88 & 7,53 & 10,30 & 1,33 & 6,37 & 5,10 & 0,00 \\
\hline 2010 & 8,81 & 8,25 & 4,16 & 10,47 & 0,73 & 7,00 & 4,68 & 0,00 \\
\hline 2011 & 13,57 & 5,94 & 5,54 & 6,78 & 1,90 & 6,57 & 5,91 & 0,00 \\
\hline 2012 & 9,12 & 2,56 & 2,20 & 2,71 & 1,45 & 2,72 & 4,97 & 0,47 \\
\hline 2013 & 9,66 & 3,82 & 2,78 & 3,72 & 2,69 & 3,95 & 5,80 & 1,13 \\
\hline 2014 & 10,54 & 1,28 & 2,72 & 1,28 & 2,34 & 1,21 & 5,33 & 1,39 \\
\hline 2015 & 8,11 & 0,42 & 2,09 & 0,42 & 2,56 & 0,85 & 5,12 & 1,70 \\
\hline 2016 & 9,47 & 2,28 & 2,47 & 2,28 & 1,88 & 0,24 & 5,37 & 0,11 \\
\hline 2017 & 9,25 & 1,06 & 3,93 & 1,06 & 2,02 & 0,80 & 6,47 & 1,47 \\
\hline Media & 10,79 & 4,83 & 4,15 & 5,13 & 1,97 & 3,90 & 5,11 & 0,70 \\
\hline
\end{tabular}

Note: $200=$ scenario parallelo dei +200 punti base; $-200=$ scenario parallelo dei -200 punti base; $99^{\circ}=$ scenario del $99^{\circ}$ percentile; $1^{\circ}=$ scenario del $1^{\circ}$ percentile; SRU = scenario dello short rates shock up; SRD = scenario dello short rates shock down; STEEP = scenario steepener; FLAT = scenario flattener . Fonte: Elaborazioni dell'Autore su dati di bilancio al 31/12 di ogni anno e su dati Datastream.

Le evidenze empiriche sull'intero periodo oggetto di analisi mostrano, in termini di valori medi, che per le banche esposte a variazioni in aumento dei tassi di interesse lo scenario parallelo dei +200 punti base conduce ad un livello medio dell'indicatore di rischio più elevato pari al $10,79 \%$ rispetto al $99^{\circ}$ percentile e allo short rates shock up, che presentano, invece, valori medi più bassi pari, rispettivamente, a $4,15 \%$ e all' $1,97 \%$. Il dato relativo allo short rates shock up risente, tuttavia, della bassa propensione delle banche commerciali italiane ad essere esposte a tale tipologia di scenario per via della particolare struttura per scadenza delle posizioni nette. È interessante osservare che lo scenario parallelo dei +200 punti base presenta un valore medio dell'indicatore di rischio più elevato dello scenario corrispondente al $99^{\circ}$ percentile e allo short rates shock up in tutti i singoli anni oggetto di analisi. L'ampiezza delle variazioni associate ai vari scenari considerati è alla base di tali evidenze. Nello specifico, lo scenario parallelo dei +200 punti base presenta, per tutte le fasce temporali della matrice per scadenza e data di riprezzamento, variazioni dei key-rates di maggiore ampiezza soprattutto sulle fasce temporali a lungo termine. In corrispondenza di tale scenario, infatti, la riduzione del valore economico associato alle posizioni nette positive sulle fasce temporali lungo termine impatta sull'esposizione al rischio in maniera più accentuata dell'incremento di valore economico registrato in corrispondenza delle posizioni nette negative sulle fasce temporali a medio termine rispetto a quanto avviene per lo scenario del $99^{\circ}$ percentile e dello short rates shock up.

In relazione, invece, alle banche esposte a variazioni in diminuzione dei tassi di interesse lo scenario del $1^{\circ}$ percentile è quello che conduce ad un livello medio dell'indicatore più alto pari al 5,13\%. I valori sono di poco superiori a quelli ottenuti a seguito dell'applicazione dello scenario parallelo dei -200 punti base e dello short rates shock down pari, rispettivamente, al $4,83 \%$ e al 3,90\%. È interessante osservare che le differenze tra i tre scenari considerati sono meno accentuate rispetto a quanto osservato per le banche esposte a variazioni in aumento. In tale contesto, inoltre, l'attivazione del vincolo di non negatività ha condotto, a partire dal 2009, ad un progressivo allineamento dell'incremento di valore economico dei fondi propri sulle fasce a breve e medio termine. Di conseguenza le differenze in termini di esposizione al rischio sono state, in larga 
parte, riconducibili all'impatto dei singoli scenari sulle fasce temporali a lungo termine dove si registra un incremento di valore economico data la presenza di posizione nette positive. Inoltre, a partire dal 2014 si sono registrate variazioni analoghe su tutte le fasce temporali della matrice per scadenza e per data di riprezzamento in corrispondenza dello scenario dei -200 punti base e del $1^{\circ}$ percentile, che hanno condotto, di conseguenza, ad uno stesso livello dell'indicatore di rischio.

Infine, per quel che concerne lo steepener si registra un valore medio sull'intero periodo oggetto di analisi dell'indicatore di rischio pari al 5,11\%. L'ampiezza delle variazioni unitamente alla progressiva applicazione del vincolo di non negatività, che ha smorzato, di fatto, l'impatto delle variazioni negative sulle fasce temporali a breve e medio termine è alla base delle evidenze ottenute. Nello specifico si rileva una elevata differenza rispetto al valore medio ottenuto in corrispondenza dello scenario parallelo dei +200 punti base pari al 10,79\% mentre le distanze rispetto ai valori medi ottenuti in corrispondenza degli altri scenari sono più contenute. Il dato ottenuto per lo steepener è rappresentativo della quasi totalità del campione data l'elevata propensione delle banche commerciali italiane ad essere esposte a tale scenario. Lo steepener, infatti, come detto nel corso della trattazione, conduce sia sulle fasce a medio termine, con eccezione di quella da oltre 4 anni a 5 anni, sia su quelle a lungo termine ad un incremento di valore economico dei fondi propri data la particolare struttura per scadenza delle posizioni nette delle banche commerciali italiane. Le evidenze relative, invece, all'applicazione del flattener mostrano un valore medio dell'indicatore di rischio abbastanza contenuto che si attesta a $0,70 \%$. Il dato risente, tuttavia, della bassa propensione delle banche commerciali italiane ad essere esposte a tale tipologia di scenario per via della particolare struttura per scadenza delle posizioni nette come evidenziato nel corso della trattazione.

\subsection{Le evidenze empiriche relative all'approccio del margine di interesse}

La seguente Tabella 7 riporta, nel corso del periodo di riferimento, il numero di banche esposte nell'ambito dell'approccio del margine di interesse ai vari scenari di variazione dei tassi di interesse considerati nella presente analisi e rappresentati da quelli attualmente in vigore e quelli proposti dal Comitato di Basilea nel 2016 e recepiti a luglio 2017 dalle linee guida EBA. Cosi come fatto nella precedente Tabella 5 in corrispondenza dei suddetti scenari di variazione dei tassi di interesse la Tabella 7 riporta i casi di neutralità al rischio (banche non esposte a due scenari considerati in maniera congiunta) e di doppia esposizione (banche esposte a entrambi i due scenari considerati in maniera congiunta).

Tabella 7. Numero di banche esposte in corrispondenza dei vari scenari di variazione dei tassi di interesse (approccio del margine di interesse)

\begin{tabular}{|c|c|c|c|c|c|c|c|c|c|c|c|c|c|c|c|c|}
\hline & $(+200)$ & $(-200)$ & $\mathbf{N}$ & D & $99^{\circ}$ & $1^{\circ}$ & $\mathbf{N}$ & D & SRU & SRD & $\mathbf{N}$ & D & STEEP & FLAT & $\mathbf{N}$ & D \\
\hline 2006 & 25 & 105 & 0 & 0 & 22 & 105 & 0 & 3 & 24 & 106 & 0 & 0 & 106 & 22 & 0 & 2 \\
\hline 2007 & 8 & 122 & 0 & 0 & 8 & 122 & 0 & 0 & 9 & 121 & 0 & 0 & 121 & 9 & 0 & 0 \\
\hline 2008 & 5 & 125 & 0 & 0 & 5 & 125 & 0 & 0 & 5 & 125 & 0 & 0 & 125 & 5 & 0 & 0 \\
\hline 2009 & 10 & 116 & 4 & 0 & 10 & 115 & 4 & 1 & 10 & 115 & 4 & 1 & 115 & 10 & 4 & 1 \\
\hline 2010 & 4 & 126 & 0 & 0 & 4 & 126 & 0 & 0 & 4 & 126 & 0 & 0 & 126 & 4 & 0 & 0 \\
\hline 2011 & 10 & 108 & 11 & 1 & 10 & 108 & 11 & 1 & 10 & 108 & 11 & 1 & 112 & 10 & 7 & 1 \\
\hline 2012 & 6 & 116 & 6 & 2 & 5 & 115 & 7 & 3 & 6 & 116 & 6 & 2 & 116 & 6 & 6 & 2 \\
\hline 2013 & 9 & 116 & 1 & 4 & 10 & 116 & 0 & 4 & 9 & 116 & 1 & 4 & 116 & 10 & 0 & 4 \\
\hline 2014 & 2 & 111 & 9 & 8 & 2 & 109 & 9 & 10 & 2 & 109 & 9 & 10 & 109 & 2 & 9 & 10 \\
\hline 2015 & 12 & 0 & 118 & 0 & 15 & 0 & 115 & 0 & 12 & 0 & 118 & 0 & 0 & 11 & 118 & 0 \\
\hline 2016 & 11 & 0 & 119 & 0 & 14 & 0 & 116 & 0 & 11 & 0 & 119 & 0 & 0 & 11 & 119 & 0 \\
\hline 2017 & 9 & 0 & 121 & 0 & 23 & 0 & 107 & 0 & 9 & 0 & 121 & 0 & 0 & 9 & 121 & 0 \\
\hline
\end{tabular}

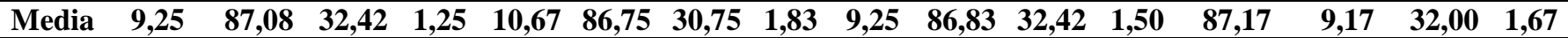

Note: $200=$ scenario parallelo dei +200 punti base; $-200=$ scenario parallelo dei -200 punti base; $99^{\circ}=$ scenario del $99^{\circ}$ percentile; $1^{\circ}=$ scenario del $1^{\circ}$ percentile; SRU = scenario dello short rates shock up; SRD = scenario dello short rates shock down; STEEP = scenario steepener ; FLAT $=$ scenario flattener . $\mathrm{N}$ e D indicano, rispettivamente, il numero di banche neutrali al rischio e esposte ad entrambi gli scenari in corrispondenza di ogni scenario congiunto.

Fonte: Elaborazioni dell'Autore su dati di bilancio al 31/12 di ogni anno e su dati Datastream.

La misurazione dell'esposizione al rischio di tasso di interesse del portafoglio bancario utilizza i dati relativi alle posizioni nette (o gap periodali) relativi alle fasce temporali temporali fino ad 1 anno coincidenti con il gapping period. In tali fasce temporali sono allocate tutte le poste a tasso variabile nonché quelle in scadenza nell'orizzonte temporale in questione. Nello specifico, dal lato dell'attivo i mutui a tasso variabile sono classificati per l'intero ammontare del debito residuo nella fascia temporale corrispondente prima alla data di revisione del tasso di interesse. I mutui basati su un meccanismo di indicizzazione il primo giorno del mese sono, in genere, allocati nella fascia temporale a vista. Un altro aggregato di particolare rilevanza nei bilanci della banche dal lato dell'attivo è rappresentato dati titoli di stato a tasso variabile e da quelli indicizzati all'inflazione che sono allocati nelle varie fasce temporali a breve termine in relazione alla data di revisione del tasso di interesse. In tal caso un meccanismo di indicizzazione su base, ad esempio, semestrale determina un'allocazione del titolo nella fascia temporale corrispondente alla distanza tra la data di valutazione dell'esposizione al rischio e la prima data 
successiva in cui è prevista la revisione del relativo tasso di interesse. A ciò si aggiungono le quote capitale dei mutui a tasso fisso in scadenza nell'orizzonte temporale di breve termine.

Dal lato del passivo nelle fasce temporali fino ad 1 anno della matrice per scadenza e per data di riprezzamento è allocata tutta la raccolta istituzionale (garantita e non) con controparti finanziarie che ha, in genere, durate di breve termine, $\mathrm{i}$ depositi e le obbligazioni in scadenza nei successivi 12 mesi la data di valutazione dell'esposizione al rischio e la parte della componente a vista distribuita in base al criterio regolamentare attualmente in uso in tali fasce temporali. Quanto detto fa sì che, in generale, la posizione netta nella fascia temporale a vista assume un valore positivo, dato il forte peso dei bilanci delle banche italiane dei mutui a tasso variabile, quelle da 1 a 6 mesi valori sia positivi sia negativi a seconda della particolare struttura per scadenza della specifica banca e, infine, quella da 6 mesi ad 1 anno valori negativi per via della quota di depositi a vista allocata in tale fascia temporale sulla base del criterio regolamentare.

In base alla (2.) in corrispondenza di ogni fascia temporale la posizione netta (o gap periodole) è la variabile che lega la variazione attesa del margine di interesse allo shock di tasso di interesse ipotizzato. Le posizioni nette negative danno luogo ad un incremento (riduzione) del margine di interesse a seguito di una diminuzione (aumento) del tasso di interesse associato alla relativa fascia temporale. Al contrario le posizioni nette positive danno luogo ad una incremento (riduzione) del margine di interesse a seguito di un aumento (diminuzione) del tasso di interesse associato alla relativa fascia temporale. Inoltre, una data variazione dei tassi di interessi esercita i propri effetti nell'intervallo di tempo intercorrente tra il punto medio della relativa fascia temporale e la fine del gapping period. Ne consegue che l'impatto delle varie posizioni nette sulla complessiva esposizione al rischio dipende non solo dalla dimensione delle stesse posizioni nette e dell'ampiezza delle variazioni dei tassi di interesse applicate ma anche dalla distanza temporale (numero di mesi) intercorrente tra la data di scadenza o di revisione del tasso, ipotizzata nel modello pari al punto medio della relativa fascia temporale, e la fine del gapping period. Sulla base di quanto detto, le posizioni nette positive sulla fascia a vista riconducibili al forte peso dei mutui a tasso variabile presenti nei bilanci della banche hanno, quindi, un forte impatto sulla complessiva esposizione al rischio di una banca nell'ambito dell'approccio del margine di interesse.

Le evidenze empiriche mostrano una maggiore presenza di banche esposte a variazioni in diminuzione dei tassi di interesse. In termini di valori medi sull'intero periodo si registrano, infatti, 87,08 banche esposte allo scenario dei -200 punti base; 86,75 allo scenario del $1^{\circ}$ percentile e 86,83 allo scenario dello short rates shock down. Le banche esposte a variazioni in aumento dei tassi di interesse sono decisamente minori. In termini di valori medi sull'intero periodo si registrano, infatti, 9,25 banche esposte alo scenario dei +200 punti base, 10,67 allo scenario del $99^{\circ}$ percentile e 9,25 allo scenario dello short rates shock up. Le ragioni alla base di tali evidenze risiedono, come sopra detto, nel forte peso, dato l'orizzonte temporale di riferimento pari ad 1 anno, dei prestiti a tasso variabile sulla complessiva esposizione al rischio. I fenomeni di neutralità al rischio nell'ambito dell'approccio del margine di interesse sono abbastanza contenuti fino al 2014 rispetto a quanto osservato nell'ambito dell'approccio del valore economico. Si registrano, invece, elevati fenomeni di neutralità negli ultimi tre anni oggetto di analisi in corrispondenza di tutti e tre gli scenari congiunti sopra menzionati. Quanto detto è riconducibile al livello negativo dei tassi di interesse nelle fasce temporali fino ad 1 anno. La conseguente applicazione del vincolo di non negatività fa sì che lo scenario di variazione in diminuzione dei tassi di interesse da applicare per tutti e tre i suddetti scenari congiunti sopra menzionati è pari a zero su tutte le fasce temporali considerate. Ciò determina, di conseguenza, la non esposizione delle banche a tali scenari. Si tratta di banche esposte, data la propria struttura delle poste di bilancio, nel caso di non applicazione del vincolo di non negatività a variazioni in diminuzione dei tassi di interesse. Negli ultimi 3 anni oggetto di analisi il numero di banche esposte a variazioni in diminuzione dei tassi di interesse nell'ambito dell'approccio del margine di interesse è in base alle ipotesi metodologiche descritte nel corso della trattazione pari a zero.

In generale, valgono considerazioni sostanzialmente analoghe a quelle effettuate nell'ambito dell'approccio del valore economico: per gli scenari congiunti simmetrici rappresentati dal +/-200 punti base e dallo short rates shock up e down l'applicazione del vincolo di non negatività è una condizione necessaria al verificarsi del fenomeno della neutralità al rischio. Nell'ambito del metodo dei percentili, invece, specifiche combinazioni di posizioni nette e variazioni dei tassi di interesse potrebbero condurre a fenomeni della neutralità al rischio anche a prescindere dall'applicazione del vincolo di non negatività.

Le evidenze empiriche mostrano, inoltre, nel corso degli anni una elevata presenza di banche esposte allo steepener, che, come noto, è caratterizzato da variazioni negative nelle fasce temporali fino ad 1 anno coincidenti con il gapping period. In termini di valori medi sull'intero periodo oggetto di analisi il numero di banche esposte a tale scenario risulta pari a 87,17 . Più contenuto è, invece, il numero di banche esposte al flattener, che, come noto, è caratterizzato da variazioni positive nelle fasce temporali fino ad 1 anno coincidenti con il gapping period. In termini di valori medi sull'intero periodo oggetto di analisi il numero di banche esposte a tale scenario risulta pari a 9,17.

Il livello negativo dei tassi di interesse negli ultimi tre anni del periodo oggetto di analisi fa sì che si verifichino, come per gli altri scenari congiunti analizzati in precedenza, elevati fenomeni di neutralità e un numero di banche di banche esposte allo steepener pari a zero. In generale valgono le stesse considerazioni fatte in precedenza in quanto nell'orizzonte 
temporale di 1 anno lo steepener e il flattener sono caratterizzati da variazioni dello stesso segno, rispettivamente, negative e positive.

Quanto alla dimensione degli impatti la successiva Tabella 8 riporta, in corrispondenza dei vari scenari di variazione dei tassi di interesse per le sole banche esposte, il rapporto tra la variazione del margine di interesse ottenuta a seguito dell'applicazione della (2.) e il livello dei fondi propri. Ciò al fine di poter avere un confronto omogeneo con le evidenze ottenute nell'ambito dell'approccio del valore economico, di cui alla precedente Tabella 6.

In tal caso i valori sono espressi con segno negativo in quanto, come sottolineato nel corso della trattazione un valore negativo ottenuto a seguito dell'applicazione della (2.) corrisponde ad una effettiva riduzione del margine di interesse a differenza di quanto avviene, invece, nell'ambito dell'approccio del valore economico dove un valore positivo ottenuto a seguito dell'applicazione della (1.) corrisponde ad una riduzione di valore economico dei fondi propri.

Tabella 8. Livello medio dell'indicatore di rischio delle banche esposte in corrispondenza dei vari scenari di variazione dei tassi di interesse (approccio del margine di interesse)

(Valori percentuali)

\begin{tabular}{|c|c|c|c|c|c|c|c|c|}
\hline Anno & $(+200)$ & $(-200)$ & $99^{\circ}$ & $\mathbf{1}^{\circ}$ & SRU & SRD & STEEP & FLAT \\
\hline 2006 & $-3,85$ & $-4,73$ & $-2,46$ & $-4,42$ & $-4,80$ & $-5,96$ & $-3,84$ & $-3,79$ \\
\hline 2007 & $-1,42$ & $-4,52$ & $-0,97$ & $-3,05$ & $-1,50$ & $-5,78$ & $-3,78$ & $-1,18$ \\
\hline 2008 & $-1,10$ & $-4,87$ & $-0,75$ & $-3,84$ & $-1,29$ & $-5,78$ & $-4,04$ & $-1,02$ \\
\hline 2009 & $-1,33$ & $-0,97$ & $-0,83$ & $-0,97$ & $-1,40$ & $-0,97$ & $-0,97$ & $-1,09$ \\
\hline 2010 & $-0,80$ & $-2,30$ & $-0,55$ & $-2,30$ & $-0,99$ & $-2,30$ & $-2,32$ & $-0,79$ \\
\hline 2011 & $-2,78$ & $-1,83$ & $-1,86$ & $-1,83$ & $-3,37$ & $-1,83$ & $-1,84$ & $-2,68$ \\
\hline 2012 & $-1,13$ & $-0,28$ & $-0,53$ & $-0,28$ & $-1,39$ & $-0,28$ & $-0,28$ & $-1,10$ \\
\hline 2013 & $-1,95$ & $-1,02$ & $-0,80$ & $-1,02$ & $-2,41$ & $-1,02$ & $-1,02$ & $-1,78$ \\
\hline 2014 & $-1,81$ & $-0,35$ & $-0,83$ & $-0,35$ & $-2,09$ & $-0,35$ & $-0,35$ & $-1,73$ \\
\hline 2015 & $-3,33$ & 0,00 & $-1,49$ & 0,00 & $-4,42$ & 0,00 & 0,00 & $-3,60$ \\
\hline 2016 & $-5,21$ & 0,00 & $-0,59$ & 0,00 & $-6,75$ & 0,00 & 0,00 & $-5,45$ \\
\hline 2017 & $-1,62$ & 0,00 & $-0,27$ & 0,00 & $-2,36$ & 0,00 & 0,00 & $-1,97$ \\
\hline Media & $-2,19$ & $-1,74$ & $-1,00$ & $-1,51$ & $-2,73$ & $-2,02$ & $-1,54$ & $-2,18$ \\
\hline
\end{tabular}

Note: $200=$ scenario parallelo dei +200 punti base; $-200=$ scenario parallelo dei -200 punti base; $99^{\circ}=$ scenario del $99^{\circ}$ percentile; $1^{\circ}=$ scenario del $1^{\circ}$ percentile; SRU = scenario dello short rates shock up; SRD = scenario dello short rates shock down; STEEP = scenario steepener; FLAT = scenario flattener . Fonte: Elaborazioni dell'Autore su dati di bilancio al 31/12 di ogni anno e su dati Datastream.

Le evidenze empiriche sull'intero periodo oggetto di analisi mostrano, in termini di valori medi, che per le banche esposte a variazioni in diminuzione dei tassi di interesse lo scenario short rates shock down conduce ad un livello medio dell'indicatore di rischio più elevato pari al $-2,02 \%$ rispetto al -200 punti base e al $99^{\circ}$ percentile, che presentano, invece, valori medi più bassi pari, rispettivamente, a $-1,74 \%$ e al $-1,51 \%$.

È interessante, tuttavia, osservare che si registrano valori medi analoghi sui vari anni a partire dal 2009 a seguito della riduzione del livello dei tassi di interesse e la conseguente applicazione del vincolo di non negatività.

I valori in questione sono, inoltre, pari a 0 negli ultimi tre anni per via della non esposizione al rischio descritta precedentemente nel corso della trattazione. Le differenze sui tre scenari sono, quindi, riconducibili agli impatti registrati nei primi tre anni oggetto di analisi (dal 2006 al 2008).

L'applicazione dello steepener caratterizzato, come detto, da variazioni negative all'interno del gapping period conduce ad un livello medio del rapporto nell'intero periodo oggetto di analisi pari a $-1,54 \%$. Valori analoghi agli altri tre scenari di variazione in diminuzione dei tassi di interesse si registrano nel 2009 e dal 2012 al 2017.

Per quanto riguarda, invece, le banche esposte a variazioni in aumento dei tassi di interesse lo scenario dello short rates shock up è quello che conduce ad un livello medio più alto del rapporto pari al $-2,73 \%$. Il valore è superiore sia allo scenario parallelo dei +200 punti sia allo scenario del flattener che fanno registrare valori medi dell'indicatore sull'intero periodo pari, rispettivamente, al $-2,19 \%$ e $-2,18 \%$. Più contenuto è il livello medio del rapporto associato allo scenario del $99^{\circ}$ percentile pari a $-1,00 \%$.

I valori osservati per le banche esposte a variazioni in aumento dei tassi di interesse nell'ambito dell'approccio del margine di interesse sono, ad eccezione dello scenario associato al $99^{\circ}$ percentile, più alti dei corrispondenti scenari di variazione negativa.

Ciò è riconducibile, essenzialmente, all'ampiezza delle variazioni che nel caso di scenari in aumento sono considerate nella loro interezza a differenza di quanto accade negli scenari di variazione negativa che, a seguito dell'applicazione del vincolo di non negatività, sono dal 2010 attenuati e, successivamente, azzerati a partire dal 2015. 


\subsection{L'integrazione dei due approcci}

L'approccio del valore economico e del margine di interesse impattano sul livello di solvibilità della banca mediante differenti modalità: il primo conduce ad un requisito di capitale interno da porre a presidio del rischio in questione; il secondo ha, invece, impatti di tipo reddituale determinando minori utili o maggiori perdite e per tale via contribuisce a determinare l'ammontare dei fondi propri e, quindi, la complessiva situazione di solvibilità della Banca. Le analisi successive si basano, in linea con gli orientamenti di vigilanza prudenziale e le prassi di settore, sulle seguenti ipotesi metodologiche:

i) la misurazione dell'esposizione al rischio e, quindi, l'analisi delle eventuali modalità di integrazione tra i due approcci è effettuata in corrispondenza di ogni singolo scenario di variazione dei tassi di interesse in aumento o in diminuzione contemplati in ciascun scenario di variazione congiunto considerati nel corso della trattazione;

ii) l'approccio del valore economico, in corrispondenza di un singolo scenario di variazione dei tassi di interesse, conduce ad un requisito di capitale positivo nel caso di effettiva esposizione della banca. Il capitale da porre a presidio del rischio in questione è pari, in tal caso, esattamente alla riduzione di valore economico registrata a seguito dell'applicazione del singolo scenario di variazione dei tassi di interesse considerato. Nel caso, invece, di non esposizione della banca al singolo scenario di variazione dei tassi di interesse considerato e, quindi, di un incremento di valore economico dei fondi propri il capitale interno da porre a presidio del rischio in questione è posto pari a zero. Non è contemplata, quindi, nell'ambito dei criteri di integrazione l'ipotesi di capitale interno negativo;

iii) l'approccio del margine di interesse può condurre, invece, in corrispondenza di un singolo scenario di variazione dei tassi di interesse ad una riduzione o incremento del risultato economico di esercizio. È contemplata, quindi, in tal caso nell'ambito dei criteri di integrazione l'ipotesi sia di un incremento che di una riduzione del margine di interesse.

I criteri di integrazione devono, quindi, definire come considerare le evidenze sul margine di interesse in corrispondenza di ogni singolo scenario di variazione dei tassi di interesse dato l'eventuale capitale interno da porre a presidio del rischio in questione nell'ambito dell'approccio del valore economico che, per ipotesi, può essere positivo o pari a zero.

Le seguenti Tabelle da 9A a 9D riportano per il periodo oggetto di analisi il numero di banche esposte in corrispondenza delle possibili combinazioni tra le differenti tipologie di esposizione al rischio che una banca può presentare a seguito dell'applicazione di ogni scenario di variazione dei tassi di interesse congiunto nell'ambito dei due approcci considerati.

Ad esempio, con il simbolo AA si indica, rispettivamente, una banca esposta a variazioni in aumento dei tassi di interesse (nel caso della Tabella 9A lo scenario parallelo dei +200 punti base) sia nell'approccio del valore economico sia in quello del margine di interesse. Con il simbolo AL si indica, invece, una banca esposta a variazioni in aumento dei tassi di interesse nell'ambito dell'approccio del valore economico (nel caso della Tabella 9A lo scenario parallelo dei +200 punti base) e a variazioni in diminuzione dei tassi di interesse nell'ambito dell'approccio del margine di interesse (nel caso della Tabella 9A lo scenario parallelo dei -200 punti base). In definitiva il simbolo indica con la prima lettera la tipologia di esposizione al rischio nell'ambito dell'approccio del valore economico e con la seconda lettera la tipologia di esposizione al rischio nell'ambito del margine di interesse.

Tabella 9A. Scenario parallelo - banche esposte differenti combinazioni

\begin{tabular}{|c|c|c|c|c|c|c|c|c|c|c|c|c|c|c|c|c|}
\hline & $\mathbf{A A}$ & $\mathbf{A L}$ & LA & $\mathbf{L L}$ & $\mathbf{A N}$ & $\mathbf{L N}$ & NA & NL & $\mathbf{N N}$ & AD & LD & DA & DL & ND & DD & DN \\
\hline 2006 & 21 & 22 & 4 & 83 & 0 & 0 & 0 & 0 & 0 & 0 & 0 & 0 & 0 & 0 & 0 & 0 \\
\hline 2007 & 8 & 21 & 0 & 101 & 0 & 0 & 0 & 0 & 0 & 0 & 0 & 0 & 0 & 0 & 0 & 0 \\
\hline 2008 & 5 & 19 & 0 & 106 & 0 & 0 & 0 & 0 & 0 & 0 & 0 & 0 & 0 & 0 & 0 & 0 \\
\hline 2009 & 10 & 22 & 0 & 90 & 3 & 1 & 0 & 4 & 0 & 0 & 0 & 0 & 0 & 0 & 0 & 0 \\
\hline 2010 & 4 & 19 & 0 & 106 & 0 & 0 & 0 & 1 & 0 & 0 & 0 & 0 & 0 & 0 & 0 & 0 \\
\hline 2011 & 10 & 23 & 0 & 76 & 8 & 3 & 0 & 9 & 0 & 0 & 0 & 0 & 0 & 1 & 0 & 0 \\
\hline 2012 & 5 & 47 & 0 & 20 & 6 & 0 & 1 & 49 & 0 & 2 & 0 & 0 & 0 & 0 & 0 & 0 \\
\hline 2013 & 9 & 59 & 0 & 22 & 1 & 0 & 0 & 35 & 0 & 3 & 1 & 0 & 0 & 0 & 0 & 0 \\
\hline 2014 & 2 & 52 & 0 & 15 & 6 & 1 & 0 & 44 & 2 & 8 & 0 & 0 & 0 & 0 & 0 & 0 \\
\hline 2015 & 11 & 0 & 0 & 0 & 56 & 6 & 1 & 0 & 55 & 0 & 0 & 0 & 0 & 0 & 0 & 0 \\
\hline 2016 & 8 & 0 & 0 & 0 & 49 & 2 & 3 & 0 & 68 & 0 & 0 & 0 & 0 & 0 & 0 & 0 \\
\hline 2017 & 8 & 0 & 0 & 0 & 49 & 1 & 1 & 0 & 71 & 0 & 0 & 0 & 0 & 0 & 0 & 0 \\
\hline Media & 8,42 & 23,67 & $\mathbf{0 , 3 3}$ & 51,58 & 14,83 & 1,33 & 0,50 & 11,83 & 16,25 & 1,08 & 0,08 & $\mathbf{0 , 0 0}$ & $\mathbf{0 , 0 0}$ & 0,08 & $\mathbf{0 , 0 0}$ & 0,00 \\
\hline
\end{tabular}


Tabella 9B. Metodo dei percentili - banche esposte differenti combinazioni

\begin{tabular}{|c|c|c|c|c|c|c|c|c|c|c|c|c|c|c|c|c|}
\hline & $\mathbf{A A}$ & $\mathbf{A L}$ & LA & $\mathbf{L L}$ & $\mathbf{A N}$ & LN & NA & NL & $\mathbf{N N}$ & AD & LD & DA & DL & ND & DD & DN \\
\hline 2006 & 18 & 14 & 4 & 91 & 0 & 0 & 0 & 0 & 0 & 3 & 0 & 0 & 0 & 0 & 0 & 0 \\
\hline 2007 & 8 & 14 & 0 & 107 & 0 & 0 & 0 & 1 & 0 & 0 & 0 & 0 & 0 & 0 & 0 & 0 \\
\hline 2008 & 5 & 11 & 0 & 106 & 0 & 0 & 0 & 8 & 0 & 0 & 0 & 0 & 0 & 0 & 0 & 0 \\
\hline 2009 & 9 & 9 & 0 & 105 & 3 & 1 & 0 & 1 & 0 & 0 & 1 & 1 & 0 & 0 & 0 & 0 \\
\hline 2010 & 4 & 8 & 0 & 117 & 0 & 0 & 0 & 0 & 0 & 0 & 0 & 0 & 1 & 0 & 0 & 0 \\
\hline 2011 & 10 & 16 & 0 & 91 & 6 & 4 & 0 & 1 & 1 & 0 & 1 & 0 & 0 & 0 & 0 & 0 \\
\hline 2012 & 4 & 23 & 0 & 21 & 7 & 0 & 1 & 71 & 0 & 3 & 0 & 0 & 0 & 0 & 0 & 0 \\
\hline 2013 & 10 & 30 & 0 & 28 & 0 & 0 & 0 & 58 & 0 & 3 & 1 & 0 & 0 & 0 & 0 & 0 \\
\hline 2014 & 2 & 24 & 0 & 15 & 6 & 1 & 0 & 70 & 2 & 10 & 0 & 0 & 0 & 0 & 0 & 0 \\
\hline 2015 & 12 & 0 & 0 & 0 & 24 & 6 & 3 & 0 & 85 & 0 & 0 & 0 & 0 & 0 & 0 & 0 \\
\hline 2016 & 13 & 0 & 0 & 0 & 74 & 2 & 1 & 0 & 40 & 0 & 0 & 0 & 0 & 0 & 0 & 0 \\
\hline 2017 & 20 & 0 & 1 & 0 & 81 & 2 & 2 & 0 & 24 & 0 & 0 & 0 & 0 & 0 & 0 & 0 \\
\hline
\end{tabular}

Note: A = banca esposta allo scenario di variazioni in aumento dei tassi di interesse; $\mathrm{L}=$ banca esposta allo scenario di variazione in diminuzione dei tassi di interesse; $\mathrm{N}=$ banca neutrale al rischio; $\mathrm{D}=$ banca esposte ad entrambi gli scenari di variazione in aumento e in diminuzione. Le differenti combinazioni riportate nella prima riga indicano la tipologia di esposizione nell'ambito, rispettivamente, dell'approccio del valore economico e del margine di interesse.

Fonte: Elaborazioni dell'Autore su dati di bilancio al 31/12 di ogni anno e su dati Datastream.

Tabella 9C. Short rate up e down - banche esposte differenti combinazioni

\begin{tabular}{|c|c|c|c|c|c|c|c|c|c|c|c|c|c|c|c|c|}
\hline & $\mathbf{A A}$ & AL & LA & $\mathbf{L L}$ & $\mathbf{A N}$ & $\mathbf{L N}$ & NA & NL & NN & AD & LD & DA & DL & ND & DD & DN \\
\hline 2006 & 7 & 3 & 17 & 103 & 0 & 0 & 0 & 0 & 0 & 0 & 0 & 0 & 0 & 0 & 0 & 0 \\
\hline 2007 & 3 & 3 & 6 & 118 & 0 & 0 & 0 & 0 & 0 & 0 & 0 & 0 & 0 & 0 & 0 & 0 \\
\hline 2008 & 2 & 1 & 3 & 124 & 0 & 0 & 0 & 0 & 0 & 0 & 0 & 0 & 0 & 0 & 0 & 0 \\
\hline 2009 & 6 & 2 & 3 & 111 & 0 & 4 & 1 & 1 & 0 & 0 & 1 & 0 & 1 & 0 & 0 & 0 \\
\hline 2010 & 1 & 1 & 2 & 125 & 0 & 0 & 1 & 0 & 0 & 0 & 0 & 0 & 0 & 0 & 0 & 0 \\
\hline 2011 & 8 & 0 & 2 & 107 & 0 & 11 & 0 & 0 & 0 & 0 & 1 & 0 & 1 & 0 & 0 & 0 \\
\hline 2012 & 4 & 4 & 1 & 104 & 2 & 2 & 0 & 8 & 2 & 2 & 0 & 1 & 0 & 0 & 0 & 0 \\
\hline 2013 & 8 & 1 & 1 & 111 & 1 & 0 & 0 & 4 & 0 & 3 & 1 & 0 & 0 & 0 & 0 & 0 \\
\hline 2014 & 2 & 2 & 0 & 71 & 2 & 4 & 0 & 36 & 3 & 7 & 0 & 0 & 0 & 2 & 1 & 0 \\
\hline 2015 & 8 & 0 & 2 & 0 & 1 & 52 & 1 & 0 & 64 & 0 & 0 & 1 & 0 & 0 & 0 & 1 \\
\hline 2016 & 5 & 0 & 0 & 0 & 0 & 7 & 6 & 0 & 112 & 0 & 0 & 0 & 0 & 0 & 0 & 0 \\
\hline 2017 & 5 & 0 & 1 & 0 & 1 & 53 & 3 & 0 & 67 & 0 & 0 & 0 & 0 & 0 & 0 & 0 \\
\hline & 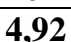 & 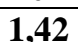 & - & 1,17 & 0.58 & 11,0 & 1.00 & 08 & 20.67 & 100 & 0.25 & 0,17 & 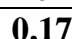 & 0,17 & 8 & 008 \\
\hline
\end{tabular}

Note: A = banca esposta allo scenario di variazioni in aumento dei tassi di interesse; $\mathrm{L}=$ banca esposta allo scenario di variazione in diminuzione dei tassi di interesse; $\mathrm{N}$ = banca neutrale al rischio; $\mathrm{D}=$ banca esposte ad entrambi gli scenari di variazione in aumento e in diminuzione. Le differenti combinazioni riportate nella prima riga indicano la tipologia di esposizione nell'ambito, rispettivamente, dell'approccio del valore economico e del margine di interesse.

Fonte: Elaborazioni dell'Autore su dati di bilancio al 31/12 di ogni anno e su dati Datastream.

Tabella 9D. Steepener e flattener - banche esposte differenti combinazioni

\begin{tabular}{|c|c|c|c|c|c|c|c|c|c|c|c|c|c|c|c|c|}
\hline & SS & SF & FS & FF & SN & FN & NS & NF & $\mathbf{N N}$ & SD & FD & DS & DF & ND & DD & DN \\
\hline 2006 & 103 & 22 & 1 & 0 & 0 & 0 & 2 & 0 & 0 & 2 & 0 & 0 & 0 & 0 & 0 & 0 \\
\hline 2007 & 116 & 8 & 2 & 0 & 0 & 0 & 2 & 0 & 0 & 0 & 0 & 1 & 1 & 0 & 0 & 0 \\
\hline 2008 & 124 & 5 & 0 & 0 & 0 & 0 & 1 & 0 & 0 & 0 & 0 & 0 & 0 & 0 & 0 & 0 \\
\hline 2009 & 115 & 10 & 0 & 0 & 4 & 0 & 0 & 0 & 0 & 1 & 0 & 0 & 0 & 0 & 0 & 0 \\
\hline 2010 & 124 & 4 & 0 & 0 & 0 & 0 & 2 & 0 & 0 & 0 & 0 & 0 & 0 & 0 & 0 & 0 \\
\hline 2011 & 111 & 10 & 0 & 0 & 7 & 0 & 1 & 0 & 0 & 1 & 0 & 0 & 0 & 0 & 0 & 0 \\
\hline 2012 & 116 & 5 & 0 & 0 & 6 & 0 & 0 & 0 & 0 & 2 & 0 & 0 & 1 & 0 & 0 & 0 \\
\hline 2013 & 116 & 9 & 0 & 0 & 0 & 0 & 0 & 0 & 0 & 3 & 0 & 0 & 1 & 0 & 1 & 0 \\
\hline 2014 & 107 & 2 & 0 & 0 & 9 & 0 & 1 & 0 & 0 & 7 & 0 & 1 & 0 & 0 & 3 & 0 \\
\hline 2015 & 0 & 9 & 0 & 0 & 117 & 0 & 0 & 0 & 0 & 0 & 0 & 0 & 3 & 0 & 0 & 1 \\
\hline 2016 & 0 & 10 & 0 & 0 & 115 & 0 & 0 & 0 & 4 & 0 & 0 & 0 & 1 & 0 & 0 & 0 \\
\hline 2017 & 0 & 8 & 0 & 0 & 121 & 0 & 0 & 0 & 0 & 0 & 0 & 0 & 1 & 0 & 0 & 0 \\
\hline
\end{tabular}

Note: $\mathrm{S}=$ banca esposta allo steepener; $\mathrm{L}=$ banca esposta al flattener; $\mathrm{N}=$ banca neutrale al rischio; $\mathrm{D}=$ banca esposte sia allo steepener che al flattener. Le combinazioni riportate nella prima riga indicano la tipologia di esposizione nell'ambito, rispettivamente, dell'approccio del valore economico e del margine di interesse.

Fonte: Elaborazioni dell'Autore su dati di bilancio al 31/12 di ogni anno e su dati Datastream.

Le evidenze empiriche mostrano che nei primi tre scenari congiunti (scenario parallelo, metodo dei percentili e short rates shock up e down) la combinazione LL risulta quella maggiormente presente. Il dato medio sull'intero periodo si attesta per i tre scenari congiunti considerati, rispettivamente, a 51,58, 56,75 e 81,17. Negli ultimi tre anni oggetto di analisi non si rilevano, tuttavia, casi di combinazione LL. Ciò è riconducibile al livello negativo dei tassi di interesse e alla conseguente applicazione del vincolo di non negatività che ha condotto nell'ambito dell'approccio del margine di interesse alla non esposizione delle banche a scenari di variazione in diminuzione. Negli ultimi tre anni si registrano, invece, elevati casi 
associati alle combinazioni AN e NN nell'ambito dello scenario parallelo e del metodo dei percentili e associati alle combinazioni LN e NN nell'ambito dello scenario short rates shock up e down. I casi di neutralità al rischio in entrambi gli approcci in corrispondenza dei vari scenari di variazione congiunta rappresentati dalla combinazione NN si presentano molto raramente negli anni precedenti il 2015. I numerosi casi neutralità al rischio rilevati dal 2012 al 2014 nell'ambito dell'approccio del valore economico, ed in particolare per quel che concerne lo scenario parallelo e il metodo dei percentili, sono stati catturati nell'ambito della combinazione NL.

Più contenute sono le banche rientranti, invece, nella combinazione AA pari, rispettivamente, per i tre scenari di variazione congiunta considerati in termini di valori medi sull'intero periodo a 8,42, 9,58 e 4,92. Da segnalare, inoltre, nell'ambito dello scenario parallelo e del metodo dei percentili le evidenze concernenti la combinazione AL, che in termini di valori medi calcolati sull'intero periodo si attesta, rispettivamente, a 23,67 e 12,42. Infine per quel che concerne lo scenario congiunto steepener /flattener si registrano elevati casi della combinazione SS, che si attesta, sulla base dei dati medi dell'intero periodo, a 86,00. Negli ultimi tre anni oggetto di analisi non si registrano casi di tale combinazione. Ciò è riconducibile alle stesse ragioni sopra descritte per la casistica AA nell'ambito degli altri scenari di variazione congiunta. Si registrano, invece, negli ultimi tre anni numerosi casi della casistica SN. Le banche sono, quindi, in un contesto di tassi negativi rimaste esposte allo steepener nell'ambito dell'approccio del valore economico e, invece, caratterizzate dal fenomeno della neutralità al rischio nell'ambito del solo approccio del margine di interesse. Si segnala il dato relativo alla casistica SF con un valore medio calcolato sull'intero periodo pari a 8,50. Le altre casistiche presentano valori medi sull'intero periodo inferiori ad 1.

È interessante analizzare in corrispondenza di ogni singola combinazione le implicazioni derivanti dall'applicazione sia dello scenario caratterizzato da variazioni positive sia di quello caratterizzato da variazioni negative. Le casistiche AA e LL rappresentano banche esposte, rispettivamente, a variazioni in aumento e in diminuzione dei tassi di interesse in entrambi gli approcci. In tal caso gli impatti sul livello di solvibilità dei due approcci andrebbero, in una prospettiva totalmente integrata, considerati entrambi. Si avrebbe, in tal caso, quindi un capitale interno positivo pari alla riduzione di valore economico registrata a seguito dell'applicazione dello specifico scenario considerato e una riduzione del margine di interesse con conseguente peggioramento del profilo di redditività e, quindi, del livello dei fondi propri. Quanto sopra detto si verifica a seguito dell'applicazione dello scenario di variazione in aumento per la casistica AA e in diminuzione per la casistica LL. L'applicazione, invece, dello scenario dei tassi di interesse in diminuzione per la casistica AA e in aumento per la casistica LL conduce ad un capitale interno da porre a presidio del rischio pari a zero nell'ambito dell'approccio del valore economico e a maggiori utili e/o minori perdita e, quindi, ad un incremento del livello dei fondi propri della banca nell'ambito dell'approccio del margine di interesse.

Le casistiche AL e LA si riferiscono, invece, a banche esposte, rispettivamente a variazioni in aumento/diminuzione nell'ambito dell'approccio del valore economico e in diminuzione/aumento nell'ambito dell'approccio del margine di interesse. L'adozione di una prospettiva integrata, come quella descritta nelle precedenti casistiche AA e LL, tale da considerare nell'ambito dei due approcci per intero entrambi gli impatti derivanti dall'applicazione del singolo scenario di variazione conduce, invece, ad un effetto compensativo. Con riferimento alla casistica AL si dovrebbe, infatti, rilevare un ammontare di capitale interno da porre a presidio del rischio in questione nell'ambito dell'approccio del valore economico a seguito dell'applicazione, dello scenario di variazione positivo. L'applicazione del medesimo scenario nell'ambito dell'approccio del margine di interesse conduce, invece, ad un maggior utile/minore perdita e, quindi, ad un miglioramento dei fondi propri. Ad analoghe considerazioni si giunge a seguito dell'applicazione dello scenario di variazione in diminuzione nell'ambito della casistica LA.

Al contrario l'applicazione dello scenario di variazione in diminuzione nell'ambito della casistica AL conduce ad un capitale interno da porre a presidio del rischio in questione pari a zero e un minor livello di utili o una maggiore perdita nell'ambito dell'approccio del margine di interesse che condurrebbe ad una riduzione dei fondi propri e, quindi, del livello di solvibilità della banca. In questo caso non vi è alcun effetto compensativo data l'ipotesi metodologica di capitale interno non negativo nell'ambito dell'approccio del valore economico. L'esposizione complessiva al rischio è impattata, quindi, dalle sole evidenze concernenti l'approccio del margine di interesse. Ad analoghe considerazioni si giunge a seguito dell'applicazione dello scenario di variazione in aumento nell'ambito della casistica LA.

Le casistiche AN e LN indicano, invece, banche esposte nell'ambito dell'approccio del valore economico a variazioni, rispettivamente positive e negative e neutrali al rischio nell'ambito dell'approccio del margine di interesse. In tal caso si registra solo un ammontare di capitale interno nell'ambito dell'approccio del valore economico da porre a presidio del rischio in questione a seguito dell'applicazione dello scenario di variazione in aumento nella casista AN e in diminuzione nella casistica LN. 
L'impatto sul profilo reddituale e quindi sul livello dei fondi propri è, invece, nullo data la neutralità registrata nell'ambito dell'approccio del margine di interesse. L'applicazione, invece, dello scenario di variazione in diminuzione alla casistica $\mathrm{AN}$ e in aumento alla casistica $\mathrm{LN}$ condurrebbe ad un requisito di capitale pari a zero nell'ambito dell'approccio del valore economico ed ad un miglioramento del profilo di solvibilità della banca dati gli impatti positivi sul profilo reddituale nell'ambito dell'approccio del margine di interesse.

Le casistiche NA e NL indicano, invece, banche neutrali al rischio nell'ambito dell'approccio del valore economico ed esposte, invece, nell'ambito dell'approccio del margine di interesse, rispettivamente, a variazioni in aumento e in diminuzione dei tassi di interesse. In tali casi si registra un ammontare di capitale interno pari a zero data la presenza del fenomeno della neutralità al rischio nell'ambito dell'approccio del valore economico e un peggioramento del profilo di redditività e quindi del livello dei fondi propri nell'ambito dell'approccio del margine di interesse.

L'applicazione, invece, dello scenario di variazione negativo alla casistica NA e positivo a quella NL determinerebbe, invece, fermo restando un requisito di capitale interno pari a zero, un miglioramento del profilo reddituale e, quindi, del livello dei fondi propri.

Infine la casistica NN indica banche non esposte né nell'ambito dell'approccio del valore economico né in quello del margine di interesse dato lo specifico scenario di variazione dei tassi di interesse congiunto.

Considerazioni del tutto analoghe valgono per lo scenario congiunto composto da singoli scenari di variazione steepener e flattener. Le seguenti Tabelle da 10A a 10D riportano, per il periodo oggetto di analisi, il livello medio dell'indicatore di rischio per le sole banche esposte in corrispondenza delle casistiche di maggiore rilevanza.

Alcune casistiche sono considerate su due differenti colonne al fine di analizzare gli impatti sia dello scenario di variazione positiva (pos) sia di quello di variazione negativa (neg). Le evidenze relative all'approccio del margine di interesse concernenti l'applicazione della formula (2) sono state rapportare, come nella Tabella 6, al livello dei fondi propri.

Tabella 10A. Livello degli indicatori di rischio banche esposte - scenario parallelo (Valori percentuali)

\begin{tabular}{|c|c|c|c|c|c|c|c|c|c|c|}
\hline & & AA pos & AL pos & AL neg & LA neg & LA pos & LL neg & AN pos & LN neg & NL neg \\
\hline \multirow{2}{*}{2006} & VE & 23,05 & 6,77 & $-6,77$ & 6,46 & $-6,46$ & 8,98 & 0,00 & 0,00 & 0,00 \\
\hline & MI & $-2,65$ & 3,17 & $-3,17$ & 10,15 & $-10,15$ & $-5,15$ & 0,00 & 0,00 & 0,00 \\
\hline \multirow{2}{*}{2007} & VE & 19,61 & 7,72 & $-7,72$ & 0,00 & 0,00 & 7,89 & 0,00 & 0,00 & 0,00 \\
\hline & MI & $-1,42$ & 2,90 & $-2,90$ & 0,00 & 0,00 & $-4,86$ & 0,00 & 0,00 & 0,00 \\
\hline \multirow[b]{2}{*}{2008} & VE & 11,51 & 11,74 & $-11,74$ & 0,00 & 0,00 & 7,71 & 0,00 & 0,00 & 0,00 \\
\hline & MI & $-1,10$ & 2,87 & $-2,87$ & 0,00 & 0,00 & $-5,23$ & 0,00 & 0,00 & 0,00 \\
\hline \multirow{2}{*}{2009} & VE & 26,01 & 6,44 & $-6,61$ & 0,00 & 0,00 & 7,97 & 23,33 & $-0,13$ & 0,07 \\
\hline & MI & $-1,33$ & 3,00 & $-0,61$ & 0,00 & 0,00 & $-1,07$ & 1,60 & $-0,69$ & 0,11 \\
\hline \multirow{2}{*}{2010} & VE & 18,48 & 6,77 & $-7,01$ & 0,00 & 0,00 & 8,25 & 0,00 & $-0,48$ & 0,00 \\
\hline & MI & $-0,80$ & 2,93 & $-1,24$ & 0,00 & 0,00 & $-2,50$ & 0,00 & $-1,87$ & 0,00 \\
\hline \multirow{2}{*}{2011} & $\mathrm{VE}$ & 33,95 & 6,69 & $-10,06$ & 0,00 & 0,00 & 6,07 & 7,86 & $-1,51$ & 2,73 \\
\hline & MI & $-3,04$ & 5,04 & $-1,44$ & 0,00 & 0,00 & $-2,03$ & 2,06 & $-1,32$ & 0,10 \\
\hline \multirow{2}{*}{2012} & VE & 17,82 & 7,49 & $-11,86$ & 0,00 & 0,00 & 2,56 & 10,99 & $-3,79$ & 0,00 \\
\hline & MI & $-0,87$ & 3,35 & $-0,19$ & 0,00 & 0,00 & $-0,44$ & 1,41 & $-0,32$ & 0,00 \\
\hline \multirow{2}{*}{2013} & VE & 19,19 & 7,98 & $-13,13$ & 0,00 & 0,00 & 3,59 & 13,40 & $-3,65$ & 0,00 \\
\hline & MI & $-2,49$ & 3,16 & $-0,84$ & 0,00 & 0,00 & $-1,23$ & 0,10 & $-1,27$ & 0,00 \\
\hline \multirow{2}{*}{2014} & VE & 17,56 & 9,05 & $-8,47$ & 0,00 & 0,00 & 1,26 & 12,55 & $-2,84$ & 1,56 \\
\hline & MI & $-1,29$ & 3,05 & $-0,31$ & 0,00 & 0,00 & $-0,43$ & 1,51 & $-0,41$ & 0,09 \\
\hline \multirow{2}{*}{2015} & VE & 14,60 & 0,00 & 0,00 & 0,00 & 0,00 & 0,00 & 6,84 & 0,00 & 0,42 \\
\hline & MI & $-2,30$ & 0,00 & 0,00 & 0,00 & 0,00 & 0,00 & 3,49 & 0,00 & 0,00 \\
\hline \multirow{2}{*}{2016} & VE & 19,91 & 0,00 & 0,00 & 0,00 & 0,00 & 0,00 & 7,77 & 0,00 & 2,28 \\
\hline & MI & $-1,20$ & 0,00 & 0,00 & 0,00 & 0,00 & 0,00 & 3,46 & 0,00 & 0,00 \\
\hline \multirow[b]{2}{*}{2017} & VE & 14,04 & 0,00 & 0,00 & 0,00 & 0,00 & 0,00 & 8,47 & 0,00 & 1,06 \\
\hline & MI & $-1,20$ & 0,00 & 0,00 & 0,00 & 0,00 & 0,00 & 4,51 & 0,00 & 0,00 \\
\hline \multirow{2}{*}{\multicolumn{2}{|c|}{$\begin{array}{l}\text { VE_media } \\
\text { MI_media }\end{array}$}} & $19,64 \%$ & 5,89 & $-6,95$ & 0,54 & $-0,54$ & 4,52 & 7,60 & $-1,03$ & 0,68 \\
\hline & & $-1,64$ & 2,46 & $-1,13$ & $\mathbf{0 , 8 5}$ & $-0,85$ & $-1,91$ & $\mathbf{1 , 5 1}$ & $-0,49$ & $\mathbf{0 , 0 2}$ \\
\hline
\end{tabular}

Note: A = banca esposta allo scenario di variazioni in aumento dei tassi di interesse; $\mathrm{L}=$ banca esposta allo scenario di variazione in diminuzione dei tassi di interesse; $\mathrm{N}$ = banca neutrale al rischio; $\mathrm{D}=$ banca esposte ad entrambi gli scenari di variazione in aumento e in diminuzione. Le differenti combinazioni riportate nella prima riga indicano la tipologia di esposizione nell'ambito, rispettivamente, dell'approccio del valore economico e del margine di interesse. L'indicazione pos/neg indica l'applicazione dello scenario di variazione positiva/negativa nell'ambito dello scenario congiunto considerato.

Fonte: Elaborazioni dell'Autore su dati di bilancio al 31/12 di ogni anno e su dati Datastream. 
Tabella 10B. Livello degli indicatori di rischio banche esposte - metodo dei percentili

(Valori percentuali)

\begin{tabular}{|c|c|c|c|c|c|c|c|c|c|c|}
\hline & & AA pos & AL pos & AL neg & LA neg & LA pos & LL neg & AN pos & LN neg & NL neg \\
\hline \multirow{2}{*}{2006} & $\mathrm{VE}$ & 9,72 & 3,18 & $-5,46$ & 6,95 & $-4,98$ & 9,03 & 0,00 & 0,00 & 0,00 \\
\hline & MI & $-1,98$ & 1,68 & $-2,80$ & 7,61 & $-6,42$ & $-4,81$ & 0,00 & 0,00 & 0,00 \\
\hline \multirow{2}{*}{2007} & VE & 7,66 & 3,08 & $-5,19$ & 0,00 & 0,00 & 8,47 & 0,00 & $-0,65$ & 0,00 \\
\hline & MI & $-0,97$ & 1,81 & $-1,80$ & 0,00 & 0,00 & $-3,23$ & 0,00 & $-1,66$ & 0,00 \\
\hline \multirow{2}{*}{2008} & VE & 4,67 & 4,75 & $-10,58$ & 0,00 & 0,00 & 5,15 & 0,00 & $-1,26$ & 0,00 \\
\hline & MI & $-0,75$ & 1,62 & $-1,84$ & 0,00 & 0,00 & $-4,12$ & 0,00 & $-2,88$ & 0,00 \\
\hline \multirow{2}{*}{2009} & $\mathrm{VE}$ & 12,11 & 3,58 & $-4,30$ & 0,00 & 0,00 & 10,42 & 8,00 & $-0,16$ & 2,69 \\
\hline & MI & $-0,93$ & 1,18 & $-0,45$ & 0,00 & 0,00 & $-1,03$ & 1,09 & $-0,16$ & 0,11 \\
\hline \multirow{2}{*}{2010} & VE & 7,42 & 3,02 & $-3,78$ & 0,00 & 0,00 & 10,55 & 0,00 & 0,00 & 0,00 \\
\hline & MI & $-0,55$ & 1,02 & $-0,67$ & 0,00 & 0,00 & $-2,43$ & 0,00 & 0,00 & 0,00 \\
\hline \multirow{2}{*}{2011} & VE & 12,82 & 2,02 & $-3,70$ & 0,00 & 0,00 & 7,00 & 2,82 & $-1,93$ & 3,26 \\
\hline & MI & $-2,03$ & 1,46 & $-1,21$ & 0,00 & 0,00 & $-1,91$ & 0,95 & $-6,15$ & 0,10 \\
\hline \multirow{2}{*}{2012} & VE & 4,19 & 1,45 & $-11,59$ & 0,00 & 0,00 & 2,71 & 2,75 & $-4,64$ & 0,00 \\
\hline & MI & $-0,46$ & 1,17 & $-0,14$ & 0,00 & 0,00 & $-0,45$ & 0,77 & $-0,29$ & 0,00 \\
\hline \multirow{2}{*}{2013} & $\mathrm{VE}$ & 4,99 & 1,93 & $-12,59$ & 0,00 & 0,00 & 3,56 & 0,00 & $-4,44$ & 0,00 \\
\hline & MI & $-1,06$ & 1,17 & $-0,71$ & 0,00 & 0,00 & $-1,27$ & 0,00 & $-1,11$ & 0,00 \\
\hline \multirow{2}{*}{2014} & VE & 4,18 & 2,23 & $-11,37$ & 0,00 & 0,00 & 1,26 & 2,31 & $-4,00$ & 1,56 \\
\hline & MI & $-0,89$ & 1,26 & $-0,31$ & 0,00 & 0,00 & $-0,43$ & 0,54 & $-0,37$ & 0,09 \\
\hline \multirow{2}{*}{2015} & VE & 3,37 & 0,00 & 0,00 & 0,00 & 0,00 & 0,00 & 1,45 & 0,00 & 0,42 \\
\hline & MI & $-1,11$ & 0,00 & 0,00 & 0,00 & 0,00 & 0,00 & 1,12 & 0,00 & 0,00 \\
\hline \multirow{2}{*}{2016} & VE & 3,81 & 0,00 & 0,00 & 0,00 & 0,00 & 0,00 & 2,23 & 0,00 & 2,28 \\
\hline & MI & $-0,51$ & 0,00 & 0,00 & 0,00 & 0,00 & 0,00 & 0,50 & 0,00 & 0,00 \\
\hline \multirow[b]{2}{*}{2017} & VE & 4,62 & 0,00 & 0,00 & 0,02 & $-2,59$ & 0,00 & 3,76 & 0,00 & 1,58 \\
\hline & MI & $-0,27$ & 0,00 & 0,00 & 0,00 & $-0,03$ & 0,00 & 0,52 & 0,00 & 0,00 \\
\hline \multicolumn{2}{|c|}{ VE_media } & 6,63 & 2,10 & $-5,71$ & $\mathbf{0 , 5 8}$ & $-0,63$ & 4,85 & 1,94 & $-1,42$ & 0,98 \\
\hline \multicolumn{2}{|c|}{ MI_media } & $-0,96$ & 1,03 & $-0,83$ & 0,63 & $-0,54$ & $-1,64$ & 0,46 & $-1,05$ & $\mathbf{0 , 0 2}$ \\
\hline
\end{tabular}

Note: $\mathrm{A}$ = banca esposta allo scenario di variazioni in aumento dei tassi di interesse; $\mathrm{L}=$ banca esposta allo scenario di variazione in diminuzione dei tassi di interesse; $\mathrm{N}$ = banca neutrale al rischio; $\mathrm{D}=$ banca esposte ad entrambi gli scenari di variazione in aumento e in diminuzione. Le differenti combinazioni riportate nella prima riga indicano la tipologia di esposizione nell'ambito, rispettivamente, dell'approccio del valore economico e del margine di interesse.

L'indicazione pos/neg indica l'applicazione dello scenario di variazione positiva/negativa nell'ambito dello scenario congiunto considerato.

Fonte: Elaborazioni dell'Autore su dati di bilancio al 31/12 di ogni anno e su dati Datastream.

Tabella 10C. Livello degli indicatori di rischio banche esposte - short rates up e down

(Valori percentuali)

\begin{tabular}{|c|c|c|c|c|c|c|c|c|c|c|}
\hline & & AA pos & AL pos & AL neg & LA neg & LA pos & LL neg & AN pos & LN neg & NL neg \\
\hline \multirow{2}{*}{2006} & VE & 3,08 & 0,69 & $-0,69$ & 2,98 & $-2,98$ & 5,70 & 0,00 & 0,00 & 0,00 \\
\hline & MI & $-4,63$ & 6,03 & $-6,03$ & 4,87 & $-4,87$ & $-5,96$ & 0,00 & 0,00 & 0,00 \\
\hline \multirow{2}{*}{2007} & VE & 2,18 & 2,37 & $-2,37$ & 2,63 & $-2,63$ & 5,58 & 0,00 & 0,00 & 0,00 \\
\hline & MI & $-2,21$ & 5,52 & $-5,52$ & 1,14 & $-1,14$ & $-5,78$ & 0,00 & 0,00 & 0,00 \\
\hline \multirow{2}{*}{2008} & VE & 1,54 & 3,28 & $-3,28$ & 1,64 & $-1,64$ & 6,47 & 0,00 & 0,00 & 0,00 \\
\hline & MI & $-0,62$ & 4,25 & $-4,15$ & 1,82 & $-1,74$ & $-5,79$ & 0,00 & 0,00 & 0,00 \\
\hline \multirow{2}{*}{2009} & VE & 1,71 & 0,52 & $-0,54$ & 2,34 & $-2,74$ & 6,66 & 0,00 & $-0,11$ & 2,74 \\
\hline & MI & $-1,86$ & 0,85 & $-0,11$ & 0,36 & $-1,04$ & $-1,00$ & 0,00 & $-0,02$ & 0,23 \\
\hline \multirow{2}{*}{2010} & VE & 1,09 & 0,37 & $-0,24$ & 1,13 & $-1,00$ & 7,09 & 0,00 & 0,00 & 0,00 \\
\hline & MI & $-0,93$ & 0,33 & $-0,22$ & 0,10 & $-1,00$ & $-2,32$ & 0,00 & 0,00 & 0,00 \\
\hline \multirow{2}{*}{2011} & VE & 2,13 & 0,00 & 0,00 & 2,56 & $-3,02$ & 7,06 & 0,00 & 0,00 & 3,39 \\
\hline & MI & $-3,88$ & 0,00 & 0,00 & 1,33 & $-2,86$ & $-1,85$ & 0,00 & 0,00 & 0,37 \\
\hline \multirow{2}{*}{2012} & $\mathrm{VE}$ & 2,70 & 0,56 & $-0,78$ & 3,20 & $-7,98$ & 2,78 & 1,08 & $-0,28$ & 0,56 \\
\hline & MI & $-1,13$ & 1,48 & $-0,08$ & 0,36 & $-3,39$ & $-0,31$ & 1,04 & $-0,13$ & 0,01 \\
\hline \multirow{2}{*}{2013} & VE & 3,17 & 1,03 & $-3,03$ & 0,61 & $-0,31$ & 3,97 & 1,27 & $-0,20$ & 0,00 \\
\hline & MI & $-3,46$ & 2,66 & $-0,97$ & 0,56 & $-0,35$ & $-1,05$ & 0,03 & $-0,83$ & 0,00 \\
\hline \multirow{2}{*}{2014} & VE & 2,51 & 0,50 & $-1,57$ & 0,00 & 0,00 & 1,22 & 0,43 & $-0,90$ & 1,24 \\
\hline & MI & $-1,90$ & 4,39 & $-0,40$ & 0,00 & 0,00 & $-0,40$ & 0,75 & $-0,29$ & 0,07 \\
\hline \multirow{2}{*}{2015} & VE & 2,96 & 0,00 & 0,00 & 2,17 & $-23,14$ & 0,00 & 0,92 & 0,00 & 0,83 \\
\hline & MI & $-2,50$ & 0,00 & 0,00 & 0,00 & $-11,37$ & 0,00 & 0,16 & 0,00 & 0,00 \\
\hline \multirow{2}{*}{2016} & VE & 1,88 & 0,00 & 0,00 & 0,00 & 0,00 & 0,00 & 0,00 & 0,00 & 0,24 \\
\hline & MI & $-2,14$ & 0,00 & 0,00 & 0,00 & 0,00 & 0,00 & 0,00 & 0,00 & 0,00 \\
\hline \multirow{2}{*}{2017} & VE & 2,26 & 0,00 & 0,00 & 0,99 & $-9,20$ & 0,00 & 0,79 & 0,00 & 0,80 \\
\hline & MI & $-2,74$ & 0,00 & 0,00 & 0,00 & $-6,17$ & 0,00 & 0,21 & 0,00 & 0,00 \\
\hline \multicolumn{2}{|c|}{ VE_media } & $2,27 \%$ & 0,78 & $-1,04$ & 1,69 & $-4,56$ & 3,88 & $\mathbf{0 , 3 8}$ & $-0,12$ & $\mathbf{0 , 8 2}$ \\
\hline \multicolumn{2}{|c|}{ MI_media } & $-2,33 \%$ & 2,12 & $-1,46$ & $\mathbf{0 , 8 8}$ & $-2,83$ & $-2,04$ & $\mathbf{0 , 1 8}$ & $-0,11$ & 0,06 \\
\hline
\end{tabular}

Note: $\mathrm{A}=$ banca esposta allo scenario di variazioni in aumento dei tassi di interesse; $\mathrm{L}=$ banca esposta allo scenario di variazione in diminuzione dei tassi di interesse; $\mathrm{N}=$ banca neutrale al rischio; $\mathrm{D}=$ banca esposte ad entrambi gli scenari di variazione in aumento e in diminuzione. Le differenti combinazioni riportate nella prima riga indicano la tipologia di esposizione nell'ambito, rispettivamente, dell'approccio del valore economico e del margine di interesse. L'indicazione pos/neg indica l'applicazione dello scenario di variazione positiva/negativa nell'ambito dello scenario congiunto considerato.

Fonte: Elaborazioni dell'Autore su dati di bilancio al 31/12 di ogni anno e su dati Datastream. 
Tabella 10D. Livello degli indicatori di rischio banche esposte - Steepener e flattener

(Valori percentuali

\begin{tabular}{|c|c|c|c|c|c|c|c|c|c|c|}
\hline & & SS steep & SF steep & SF flat & FS flat & FS steep & FF flat & SN steep & FN flat & NF flat \\
\hline \multirow{2}{*}{2006} & $\mathrm{VE}$ & 3,24 & 9,49 & $-6,77$ & 0,37 & $-0,11$ & 0,00 & 0,00 & 0,00 & 0,00 \\
\hline & MI & $-3,86$ & 3,33 & $-4,14$ & 10,66 & $-8,64$ & 0,00 & 0,00 & 0,00 & 0,00 \\
\hline \multirow{2}{*}{2007} & VE & 3,34 & 9,62 & $-7,00$ & 3,03 & $-5,94$ & 0,00 & 0,00 & 0,00 & 0,00 \\
\hline & MI & $-3,73$ & 0,85 & $-1,05$ & 8,94 & $-7,22$ & 0,00 & 0,00 & 0,00 & 0,00 \\
\hline \multirow{2}{*}{2008} & VE & 4,36 & 5,55 & $-3,84$ & 0,00 & 0,00 & 0,00 & 0,00 & 0,00 & 0,00 \\
\hline & MI & $-4,04$ & 0,81 & $-1,02$ & 0,00 & 0,00 & 0,00 & 0,00 & 0,00 & 0,00 \\
\hline \multirow{2}{*}{2009} & $\mathrm{VE}$ & 4,37 & 11,32 & $-7,60$ & 0,00 & 0,00 & 0,00 & 11,30 & 0,00 & 0,00 \\
\hline & MI & $-0,97$ & 0,54 & $-1,19$ & 0,00 & 0,00 & 0,00 & 0,23 & 0,00 & 0,00 \\
\hline \multirow{2}{*}{2010} & $\mathrm{VE}$ & 4,55 & 8,57 & $-5,81$ & 0,00 & 0,00 & 0,00 & 0,00 & 0,00 & 0,00 \\
\hline & MI & $-2,33$ & 0,27 & $-0,79$ & 0,00 & 0,00 & 0,00 & 0,00 & 0,00 & 0,00 \\
\hline \multirow{2}{*}{2011} & VE & 5,09 & 14,12 & $-9,04$ & 0,00 & 0,00 & 0,00 & 7,54 & 0,00 & 0,00 \\
\hline & MI & $-1,85$ & 1,55 & $-2,91$ & 0,00 & 0,00 & 0,00 & 0,45 & 0,00 & 0,00 \\
\hline \multirow{2}{*}{2012} & $\mathrm{VE}$ & 4,89 & 6,26 & $-4,56$ & 0,00 & 0,00 & 0,00 & 5,07 & 0,00 & 0,00 \\
\hline & MI & $-0,29$ & 0,11 & $-1,07$ & 0,00 & 0,00 & 0,00 & 0,03 & 0,00 & 0,00 \\
\hline \multirow{2}{*}{2013} & $\mathrm{VE}$ & 5,93 & 6,32 & $-3,36$ & 0,00 & 0,00 & 0,00 & 0,00 & 0,00 & 0,00 \\
\hline & MI & $-1,05$ & 0,36 & $-2,22$ & 0,00 & 0,00 & 0,00 & 0,00 & 0,00 & 0,00 \\
\hline \multirow{2}{*}{2014} & VE & 5,42 & 5,92 & $-2,66$ & 0,00 & 0,00 & 0,00 & 5,02 & 0,00 & 0,00 \\
\hline & MI & $-0,37$ & 0,00 & $-1,59$ & 0,00 & 0,00 & 0,00 & 0,05 & 0,00 & 0,00 \\
\hline \multirow{2}{*}{2015} & VE & 0,00 & 7,07 & $-6,61$ & 0,00 & 0,00 & 0,00 & 5,11 & 0,00 & 0,00 \\
\hline & MI & 0,00 & 0,00 & $-3,62$ & 0,00 & 0,00 & 0,00 & 0,00 & 0,00 & 0,00 \\
\hline \multirow{2}{*}{2016} & VE & 0,00 & 7,42 & $-7,37$ & 0,00 & 0,00 & 0,00 & 5,23 & 0,00 & 0,00 \\
\hline & MI & 0,00 & 0,00 & $-5,91$ & 0,00 & 0,00 & 0,00 & 0,00 & 0,00 & 0,00 \\
\hline \multirow{2}{*}{2017} & $\mathrm{VE}$ & 0,00 & 6,59 & $-4,73$ & 0,00 & 0,00 & 0,00 & 6,51 & 0,00 & 0,00 \\
\hline & MI & 0,00 & 0,00 & $-2,01$ & 0,00 & 0,00 & 0,00 & 0,00 & 0,00 & 0,00 \\
\hline \multirow{2}{*}{\multicolumn{2}{|c|}{$\begin{array}{l}\text { VE_media } \\
\text { MI media }\end{array}$}} & 3,43 & 8,19 & $-5,78$ & 0,28 & $-0,50$ & 0,00 & 3,81 & 0,00 & 0,00 \\
\hline & & $-1,54$ & 0,65 & $-2,29$ & 1,63 & $-1,32$ & 0,00 & 0,06 & 0,00 & 0,00 \\
\hline
\end{tabular}

Note: $\mathrm{S}=$ banca esposta allo steepener; $\mathrm{L}=$ banca esposta al flattener; $\mathrm{N}=$ banca neutrale al rischio; $\mathrm{D}=\mathrm{banca}$ esposte sia allo steepener che al flattener. $\mathrm{Le}$ differenti combinazioni riportate nella prima riga indicano la tipologia di esposizione nell'ambito, rispettivamente, dell'approccio del valore economico e del margine di interesse. L'indicazione steep/flat indica l'applicazione dello scenario di variazione steepener/flattener nell'ambito dello scenario congiunto considerato.

Fonte: Elaborazioni dell'Autore su dati di bilancio al 31/12 di ogni anno e su dati Datastream.

Le evidenze empiriche di cui alle suddette Tabelle da 10A a 10D devono essere lette tenendo presente che un valore positivo (negativo) in corrispondenza dell'approccio del valore economico (margine di interesse) corrisponde ad una esposizione della banca e, quindi, ad una riduzione del valore economico (peggioramento del profilo reddituale della banca). In altri termini ad un valore positivo nell'ambito dell'approccio del valore economico corrisponde un capitale interno positivo da porre a presidio del rischio di tasso di interesse del portafoglio bancario mentre un valore negativo nell'ambito dell'approccio del margine di interesse ad un peggioramento degli utili della banca e, quindi, del livello dei fondi propri.

I valori di cui alle casistiche AA (SS) e LL (FF) sono, quindi, per costruzione sempre positivi per il valore economico e negativi per il margine di interesse a meno che in uno specifico anno non si rilevano banche effettivamente esposte alle combinazioni in questione. In questo ultimo caso si hanno valori pari a zero. In tali casistiche l'impatto complessivo sull'esposizione al rischio della banca è dato dalla somma in valore assoluto dei due valori. In termini di evidenze medie sull'intero periodo si segnala l'elevato valore dell'indicatore di rischio registrato nell'ambito dell'approccio del valore economico nella casistica AA in corrispondenza dello scenario parallelo e pari al 19,64 rispetto altri scenari di variazione dei tassi di interesse dove si registrano valori decisamente più contenuti. L'indicatore di rischio si attesta nell'ambito del metodo dei percentili al 6,63, nello short rates shock up e down al 2,27 e nello steepener /flattener al 3,43. Nell'ambito dell'approccio del margine di interesse il valore più elevato si registra in corrispondenza dello short rates shock up e down pari al -2,33. Gli altri scenari presentano valori di poco inferiori e pari al -1,64 nello scenario parallelo, al -1,54 nello steepener /flattener e al 0,96 nel metodo dei percentili. Nell'ambito della casistica LL è, invece, il metodo dei percentili a condurre ad un maggiore livello dell'indicatore di rischio nell'ambito dell'approccio del valore economico pari al 4,85 a fronte di una riduzione del margine di -1,64. Di poco inferiore le evidenze relative allo scenario parallelo $(4,52$ e -1,91) e allo short rates shock up e down $(3,88$ e -2,04). Lo scenario congiunto steepener e flattener non presenta banche esposte alla casistica in questione. 
Le combinazioni AL (scenario positivo) e LA (scenario negativo) sono, invece, per costruzione sempre positivi in entrambi gli approcci a meno di banche non esposte. Considerazioni analoghe valgono per gli scenari SF (scenario steepener) e FS (scenario flattener). A fronte di una riduzione del valore economico dei fondi propri si rileva, quindi, un incremento del margine di interesse. L'impatto complessivo sull'esposizione al rischio della banca è dato dalla differenza in valore assoluto dei due valori. Nella casistica AL/SF lo steepener conduce nell'ambito dell'approccio del valore economico ad un livello medio sull'intero periodo dell'indicatore di rischio più elevato pari al 8,19 a fronte di un incremento del margine di interesse dello 0,65 . Gli altri scenari presentano livelli medi sull'intero periodo dell'indicatore di rischio più contenuti nell'ambito dell'approccio del valore economico e distanza minore tra i due approcci: scenario parallelo $(5,89$ e 2,46$)$, metodo dei percentili $(2,10$ e 1,03$)$ e short rates shock up e down $(0,78$ e 2,12). Il basso numero di banche esposte alle combinazioni LA (scenario negativo) e FS (scenario flattener) fa sì che le relative evidenze, anche se abbastanza contenute in termini di livelli, siano poco significative. Le stesse combinazioni AL(SF) e LA(FS) in corrispondenza degli scenari, rispettivamente, negativi (flattener) e positivi (steepener) sono, invece, per costruzione sempre negativi in entrambi gli approcci. L'impatto complessivo sull'esposizione al rischio della banca è, quindi, riconducibile, dato il capitale interno pari a zero nell'ambito dell'approccio del valore economico, alle sole evidenze riconducibili al margine di interesse. L'impatto maggiore sul margine di interesse si registra nella casistica $\mathrm{AL}(\mathrm{SF})$ a seguito dell'applicazione del flattener $(-2,29)$ e nella casistica LA(FS) a seguito dell'applicazione dello short rates shock down $(-2,83)$.

Le altre casistiche AN (SN) scenario positivo (steepener) e LN(FN) scenario negativo (flattener) presentano valori positivi sia nell'ambito dell'approccio del valore economico che del margine di interesse. L'impatto complessivo sull'esposizione al rischio della banca è, quindi, riconducibile al solo approccio del valore economico. I valori medi più elevati dell'indicatore di rischio si registrano per la casistica $\mathrm{AN}(\mathrm{SN})$ in corrispondenza dell'applicazione dello scenario parallelo dei +200 punti base $(7,60)$ e dello steepener $(3,81)$. Più contenuti i valori associati al $99^{\circ}$ percentile e allo short rates up pari, rispettivamente, all'1,94 e allo 0,38. Per quel che concerne la casistica $\mathrm{LN}(\mathrm{FN})$ si osservano valori degli indicatori di rischio molto bassi e inferiori all' 1,00 . L'esposizione delle banche a tale combinazione è, tuttavia, molto rara, ad eccezione di quanto accade nello scenario short rates shock up e down. Infine, la casistica NL(NF) scenario negativo (flattener) presenta valori negativi sia nell'ambito dell'approccio del valore economico che del margine di interesse. L'impatto sulla complessiva esposizione al rischio della banca è, quindi, riconducile al solo approccio del margine di interesse. I valori medi sull'intero periodo del relativo indicatore di rischio sono, comunque, molto bassi.

\section{La proposta metodologica di integrazione}

\subsection{Il quadro normativo}

Le recenti linee guida dell'ABE pubblicate il 19/07/2018 trattano le modalità di determinazione del capitale interno da porre a presidio del rischio di tasso di interesse del portafoglio bancario dai punti 22 a 31 . Il punto 22 fornisce delle indicazioni generali sottolineando che il capitale interno da porre a presidio del rischio in questione deve essere commisurato non solo all'effettiva esposizione al rischio ma anche al relativo obiettivo di Risk Appetite. Le evidenze devono essere, inoltre, dettagliatamente riportate in sede di stesura del Resoconto ICAAP. Il punto 23 stabilisce che le banche devono considerare sia l'approccio del valore economico sia quello del margine di interesse nell'ambito della valutazione della loro esposizione al rischio di tasso di interesse del portafoglio bancario.

Lo stesso punto 23 precisa, tuttavia, che, a tal fine, le banche non devono effettuare un "double-count" con riferimento al capital interno da porre a presidio del rischio in questione a seguito dell'applicazione dell'approccio del valore economico e del margine di interesse ${ }^{15}$.

Il successivo punto 24, inoltre, sottolinea nel dettaglio l'esigenza di porre a presidio del rischio in questione del capitale interno a seguito degli impatti che variazioni avverse dei tassi di interesse possono avere sia sul valore economico che sul profilo reddituale della banca. In relazione all'approccio del margine di interesse lo stesso punto 24 sottolinea la necessità di valutare in tale sede le relative implicazioni sul livello delle riserve di capitale ${ }^{16}$. I successivi punti (da 25 a 31 ) delle linee

${ }^{15}$ Nello specifico il punto 23 stabilisce quanto segue: Institutions should demonstrate that their internal capital is commensurate with the level of IRRBB, taking into account the impact on internal capital of potential changes in the institution's economic value and future earnings resulting from EV and earning measures from changes in interest rates. Institutions are not expected to double-count their internal capital for EV and earning measures".

${ }^{16}$ Nello specifico il punto 24 stabilisce quanto segue: In their ICAAP analysis of the amount of internal capital required for IRRBB, institutions should consider: a) internal capital held for risks to economic value that could arise from adverse movements in interest rates; and b) internal capital needs arising from the impact of rate changes on future earnings capacity, and the resultant implications for internal capital buffer levels." 
guida forniscono ulteriori principi funzionali ad una più corretta misurazione del rischio e alla conseguente calibrazione del capitale interno da porre a presidio del rischio in questione. In particolare, il paragrafo 25 stabilisce che le banche non devono basarsi unicamente sul supervisory outlier test ma sviluppare anche delle proprie metodologie facendo riferimento al proprio livello di rischio ai relativi obiettivi e a quanto riportato nelle politiche interne. I paragrafi 26 e 30 forniscono, inoltre, un elenco di elementi da tenere in considerazione nell'ambito, rispettivamente, della valutazione della propria adeguatezza patrimoniale e nella decisione di considerare o meno del capitale interno a presidio delle evidenze derivanti dall'applicazione dell'approccio del margine di interesse. Si rimanda alle stesse linee guida per un eventuale approfondimento.

Le linee guida dell'EBA non definiscono nel dettaglio, cosi come il recente aggiornamento della Circolare 285 di Banca d'Italia, le modalità di calcolo del capitale interno mediante il combinato utilizzo delle metriche di valutazione utilizzate nell'ambito dei due approcci (valore economico e margine di interesse) fornendo solo una serie di principi a cui fare riferimento nell'ambito della misurazione dell'esposizione al rischio in questione e la conseguente valutazione della propria adeguatezza di capitale. Al riguardo è interessante osservare che il documento di consultazione del Comitato di Basilea, pubblicato del giugno del 2015, proponeva quattro differenti criteri volti a determinare il requisito minimo di capitale, nell'ambito del I pilastro, da porre a presidio del rischio di tasso di interesse del portafoglio bancario. I criteri non sono stati successivamente riproposti nel documento tecnico definitivo emanato nell'aprile del 2016, presumibilmente data la collocazione del rischio in questione nell'ambito del II pilastro. La loro analisi può, tuttavia, essere funzionale ad avere una visione più ampia della questione al fine di poter definire un criterio metodologico da adottare in sede ICAAP per la combinazione dei due approcci nella valutazione complessiva dell'adeguatezza patrimoniale.

I quattro criteri si basavano su un comune principio metodologico in base al quale il requisito minimo di capitale era calcolato facendo riferimento allo scenario di variazione dei tassi di interesse più penalizzante per la banca in termini di maggiore riduzione di valore economico dei fondi propri e del margine di interesse. Nell'ambito dell'approccio del valore economico erano considerati tutti e 6 gli scenari proposti dal Comitato di Basilea; nell'ambito dell'approccio del margine di interesse i soli scenari paralleli dei +/-200 punti base. La proposta prevedeva anche ipotesi di compensazione tra le varie valute di operatività in corrispondenza di ogni singolo scenario di variazione dei tassi di interesse mediante l'applicazione di un parametro posto pari a 0,25 . Ciò al fine di attenuare, in corrispondenza dei singoli scenari di variazione dei tassi di interesse considerati, l'impatto sulla complessiva esposizione al rischio delle eventuali variazioni positive del valore economico e/o del margine di interesse per una data valuta di operatività.

Nello specifico, il criterio 1 considerava il solo approccio del valore economico e determinava il requisito minimo di capitale come la maggiore riduzione del valore economico calcolata in corrispondenza dei sei nuovi scenari di variazione dei tassi di interesse proposti dal Comitato di Basilea. I successivi criteri da 2 a 4 includevano, invece, nel calcolo del relativo requisito minimo di capitale anche l'approccio del margine di interesse. Il criterio 2 determinava il requisito minimo di capitale come il valore massimo tra le riduzioni di valore economico calcolate in corrispondenza dei sei singoli scenari di variazione dei tassi di interesse e le riduzioni del margine di interesse calcolate, invece, sui soli due scenari paralleli di +/-200 punti base. Il criterio 3 introduceva, rispetto al criterio 2, nell'ambito dell'approccio del valore economico in corrispondenza di ciascuno dei 6 scenari di variazione dei tassi di interesse un meccanismo di attenuazione nel caso le evidenze sul margine di interesse apportavano dei benefici alla banca in termini reddituali. Il requisito minimo di capitale era, quindi, calcolato come il valore massimo tra le riduzioni di valore economico calcolate sui sei nuovi scenari di variazione dei tassi di interesse compensate da eventuali benefici sul margine di interesse e le riduzioni del margine di interesse calcolate, invece, sui soli due scenari paralleli di +/-200 punti base. Infine, il criterio 4 introduceva una soglia minima calcolata a partire dai dati contabili come differenza tra il margine di interesse e i costi associati alle attività proprie del portafoglio bancario. La soglia così ottenuta, se positiva, va a compensare le riduzioni del valore di valore economico ottenute in corrispondenza dei sei nuovi scenari e le riduzioni del margine di interesse ottenute in corrispondenza dei soli scenari paralleli dei $+/-200$ punti base ai fini del calcolo del requisito minimo di capitale. Il requisito minimo era, quindi, calcolato come il valore massimo tra le riduzioni di valore ottenute nell'ambito dei due approcci (valore economico e margine di interesse) compensate con il valore della suddetta soglia. Se le evidenze ottenute erano, in valore assoluto, tutte inferiori a tale soglia non vi era, quindi, alcun requisito minimo di capitale.

In definitiva, i criteri 1 e 2 escludevano eventuali ipotesi di aggregazione e/o compensazione, in corrispondenza di ogni singolo scenario di variazione dei tassi di interesse, delle evidenze risultanti dai due approcci (valore economico e margine di interesse) ai fini della determinazione del requisito minimo di capitale. Il criterio 3, invece, contemplava un meccanismo di compensazione in corrispondenza dei singoli scenari di variazione dei tassi di interesse solo nell'ipotesi di variazioni negative del valore economico e positive del margine di interesse. Il criterio 4 consentiva, infine, di compensare le variazioni negative del valore economico e del margine di interesse ottenute in corrispondenza dei vari scenari con una proxy contabile, calcolata come differenza tra margine di interesse e costi associati al portafoglio bancario, solo se positiva. La proxy 
contabile, inoltre, era la stessa per tutti gli scenari considerati. In nessun caso era, quindi, consentito sommare, in corrispondenza dei singoli scenari di variazione dei tassi di interesse utilizzati, eventuali riduzioni del valore economico con eventuali riduzioni del margine di interesse e/o di altre misure o proxy contabili se negative. Il disposto normativo sanciva, quindi, implicitamente un principio di non aggregazione in corrispondenza dei singoli scenari di variazione dei tassi di interesse applicati di eventuali variazioni negative del valore economico e del margine di interesse. È, infine, interessante sottolineare che la compensabilità nell'ambito del principio 3 era piena: la variazione negativa di capitale interno registrata a seguito dell'applicazione di uno specifico scenario di variazione dei tassi di interesse nell'approccio del valore economico era sommata algebricamente alla riduzione del margine di interesse rilevata ai fini della quantificazione del capitale interno complessivo.

Il suddetto principio di non aggregazione potrebbe essere considerato in linea con quanto riportato nel paragrafo 23 delle recenti linee guida secondo cui "Institution are not expected to double-count their internal capital for EV and earning measures". Al riguardo, è interessante notare che nell'ambito delle risposte degli operatori che hanno partecipato alla consultazione, riportate nelle stesse linee guida, alla richiesta di maggiori delucidazioni circa il concetto di "double-couting" $\mathrm{e}$, in generale, alle modalità di integrazione tra i due approcci del valore economico e del margine di interesse l'EBA ha lasciato ampi margini di discrezionalità.

\subsection{Un possibile approccio metodologico}

Nel presente paragrafo si riporta una possibile proposta metodologica funzionale a calcolare l'impatto degli otto scenari di variazione dei tassi di interesse descritti nel corso della trattazione considerando sia l'approccio del valore economico sia quello sul margine di interesse (con un orizzonte temporale di 1 anno). La variabile obiettivo su cui misurare gli impatti dei vari scenari nei due differenti approcci è l'indicatore di solvibilità comprensivo dei rischi di I e II pilastro. In particolare, le banche nell'ambito del proprio Risk Appetite Framework $(R A F)$ inseriscono, in genere, oltre all'indicatore di solvibilità sul I pilastro (Total Capital Ratio - TCR) calcolato sulla base delle indicazioni del quadro normativo di vigilanza prudenziale anche un indicatore di solvibilità rettificato, indicato con TCRII, che tiene conto anche dei rischi di II pilastro tra cui vi rientrano il rischio di concentrazione (nelle componenti single-name e geo-settoriale) e appunto il rischio di tasso di interesse del portafoglio bancario. L'indicatore in questione è calcolato in base alla seguente formula:

$$
T C R I I=\frac{F P}{(R W A+R W A I I)}
$$

Dove FP, RWA e RWAII sono, rispettivamente, il livello dei fondi propri e le attività ponderate per i rischi di I e II pilastro. Queste ultime sono ottenute moltiplicando il relativo capitale interno per 12,5. L'inserimento di tale indicatore nei RAF delle banche si basa, in generale, su due specifiche motivazioni. Innanzitutto, esso consente alla Banca di monitorare in maniera più accurata l'effettivo ammontare di capitale libero a disposizione, che, come noto, rappresenta una delle leve strategiche funzionali alla crescita dimensionale. Il volume di nuovi crediti potenzialmente erogabili e, in generale, del massimo incremento delle attività aziendali è, infatti, un multiplo dell'effettivo capitale libero a disposizione della Banca. Inoltre, il livello di tale indicatore al 31/12 di anno riportato nel Rendiconto ICAAP è strettamente collegato ai requisiti minimi sul TCR comunicati, successivamente, dalle Autorità di vigilanza in sede SREP. Il mantenimento di un livello di TCRII sopra una determinata soglia di tolleranza, calibrata sulla base delle evidenze dell'ultimo ICAAP e SREP disponibile, consente, quindi, di orientare, in chiave prospettiva, l'operatività della banca verso specifici requisiti minimi attesi.

L'approccio metodologico proposto, di cui alla successiva Tabella 11, si basa sull'individuazione dello scenario di variazione dei tassi di interesse che ha un maggiore impatto sull'indicatore di solvibilità sopra descritto sulla base delle due seguenti ipotesi già descritte nel corso della trattazione: a) l'approccio del valore economico conduce ad un capitale interno da porre a presidio del rischio in questione in caso di riduzione del valore economico a seguito dell'applicazione dei differenti scenari di variazione dei tassi di interesse. In caso, invece, di incremento di valore economico dei fondi propri si ha un ammontare di capitale interno pari a zero; b) l'approccio del margine di interesse conduce ad un maggior/minore livello della relativa voce di conto economico che, a sua volta, impatta sul risultato di esercizio e, di conseguenza, sul livello dei fondi propri per via dei minori utili distribuiti o della maggiore perdita. In corrispondenza dei vari scenari di variazione dei tassi di interesse considerati (dalla terza colonna in poi) sono riportate le evidenze in termini di capitale interno ottenute nell'ambito dell'approccio del valore economico (prima riga) e del margine di interesse (seconda riga). È importante sottolineare che, nell'approccio del valore economico, un valore positivo sta ad indicare una riduzione del valore economico a seguito dello shock di tasso ipotizzato e, viceversa, in caso di valore negativo. Nell'ambito, invece, dell'approccio del margine di interesse, un valore positivo sta ad indicare un incremento dello stesso margine di interesse a seguito dello shock di tasso ipotizzato e, viceversa, in caso di valore negativo. 
Le evidenze in termini di valore economico sono considerate al denominatore dell'indicatore di solvibilità, comprensivo dei rischi di II pilastro nell'ambito delle attività ponderate per il rischio moltiplicando il capitale interno ottenuto a seguito dell'applicazione degli scenari di variazione dei tassi di interesse per 12,5. Le evidenze, invece, in termini di approccio del margine di interesse sono portate in aumento/riduzione dei fondi propri al netto dell'effetto fiscale. Inoltre, si ipotizza che la banca nell'ambito dei rischi di II pilastro sia esposta solo al rischio di concentrazione (nelle due componenti single-name e geo-settoriale) e al rischio di tasso di interesse del portafoglio bancario.

Nella seconda colonna della Tabella 11 è riportata la sintesi della situazione patrimoniale della banca oggetto di analisi alla data di valutazione e, nello specifico, il capitale interno calcolato sui rischi di I pilastro (credito, mercato e operativo), e quello sui rischi di secondo pilastro (rischio di concentrazione nella versione sia single-name che geo-settoriale). Il requisito sul rischio di tasso di interesse del portafoglio bancario è considerato, invece, nelle colonne successive in corrispondenza dei differenti scenari di variazione dei tassi di interesse considerati nella trattazione. Il livello dei fondi propri, di cui all'ottava riga, è considerato sia prima dell'applicazione degli impatti sul rischio di tasso di interesse del portafoglio bancario (seconda colonna) sia a seguito degli impatti dei vari scenari di variazione dei tassi di interesse oggetto di analisi (colonne successive). Il livello dell'indicatore di solvibilità sui rischi di I e II pilastro, di cui alle ultime tre righe della prima colonna, è calcolato, quindi, senza considerare l'eventuale impatto del rischio di tasso di interesse del portafoglio bancario.

Nelle ultime righe della Tabella 11 a partire dalla terza colonna è riportato: l'impatto delle sole evidenze sul valore economico (A), l'impatto delle sole evidenze sul margine di interesse (B) e l'impatto congiunto dei due approcci (C) sull'indicatore di solvibilità calcolato sui rischi di I e II pilastro, con evidenza della differenza $\left(\Delta_{-} \mathrm{A} / \mathrm{B} / \mathrm{C}\right)$ rispetto al livello calcolato senza considerare il rischio di tasso di interesse del portafoglio bancario (di cui alla seconda colonna). Le evidenze ottenute mostrano che per quel che concerne l'approccio del valore economico lo scenario più penalizzante è rappresentato dalla variazione parallela di +200 punti base che conduce l'indicatore di solvibilità dal 20,93 al 18,19 per una differenza di circa 274 punti base. Di poco inferiore è l'impatto dello Steepener in corrispondenza del quale l'indicatore di solvibilità si attesta al 18,40 per una riduzione di complessivi 252 punti base.

L'applicazione del $99^{\circ}$ percentile conduce, infine, ad un indicatore di solvibilità del 19,12 facendo registrare una riduzione di 180 punti base. Per quanto riguarda, invece, l'approccio del margine di interesse l'applicazione del vincolo di non negatività, come descritto nel corso della trattazione, fa sì che non si registri alcuna variazione in diminuzione. Gli scenari che, invece, conducono ad un incremento del margine impattano positivamente sull'indicatore di solvibilità determinandone un aumento. Di conseguenza l'ipotesi congiunta fa sì che il beneficio derivante dall'applicazione dell'approccio del margine di interesse determina una attenuazione dell'impatto dell'approccio del valore economico per lo scenario dei +200 punti base e per lo scenario del $99^{\circ}$ percentile. L'indicatore di solvibilità fa, quindi, registrare un incremento, rispettivamente dal 18,19 al 18,85 e dal 19,12 al 19,17 .

Tabella 11. Integrazione tra i due approcci: una proposta metodologica

(Valori in migliaia di euro - livello degli indicatori in valori percentuali)

\begin{tabular}{|c|c|c|c|c|c|c|c|c|c|}
\hline & & 200 & $(-200)$ & 99 & 1 & SRU & SRD & STEEP & FLATT \\
\hline Impatto sul valore economico & & 7.477 & -9.403 & 4.685 & -9.403 & -5.027 & -2.309 & 6.813 & -7.471 \\
\hline Impatto sul margine di interesse & & 4.711 & 0 & 330 & 0 & 5.823 & 0 & 0 & 4.643 \\
\hline Totale capitale interno I pilastro & 47.000 & 47.000 & 47.000 & 47.000 & 47.000 & 47.000 & 47.000 & 47.000 & 47.000 \\
\hline Rischio di concentrazione & 2.700 & 2.700 & 2.700 & 2.700 & 2.700 & 2.700 & 2.700 & 2.700 & 2.700 \\
\hline Rischio tasso di interesse & 0 & 7.477 & 0 & 4.685 & 0 & 0 & 0 & 6.813 & 0 \\
\hline Totale capitale interno II pilastro & 2.700 & 10.177 & 2.700 & 7.385 & 2.700 & 2.700 & 2.700 & 9.513 & 2.700 \\
\hline Totale capitale interno I e II pilastro & 49.700 & $\mathbf{5 7 . 1 7 7}$ & 49.700 & 54.385 & 49.700 & 49.700 & 49.700 & 56.513 & 49.700 \\
\hline Fondi Propri & 130.000 & 134.711 & 130.000 & 130.330 & 130.000 & 135.823 & 130.000 & 130.000 & 134.643 \\
\hline A. Indicatore di solvibilità (impatto VE) & 20,93 & 18,19 & 20,93 & 19,12 & 20,93 & 20,93 & 20,93 & 18,40 & 20,93 \\
\hline$\Delta \_\mathrm{A}$ & & $-2,74$ & 0,00 & $-1,80$ & 0,00 & 0,00 & 0,00 & $-2,52$ & 0,00 \\
\hline B. Indicatore di solvibilità (impatto MI) & 20,93 & 21,68 & 20,93 & 20,98 & 20,93 & 21,86 & 20,93 & 20,93 & 21,67 \\
\hline$\Delta \_\mathrm{B}$ & & $\mathbf{0 , 7 6}$ & 0,00 & $\mathbf{0 , 0 5}$ & 0,00 & 0,94 & 0,00 & $\mathbf{0 , 0 0}$ & 0,75 \\
\hline C. Ipotesi congiunta & 20,93 & 18,85 & 20,93 & 19,17 & 20,93 & 21,86 & 20,93 & 18,40 & 21,67 \\
\hline$\Delta \mathrm{C}$ & & $-2,08$ & 0,00 & $-1,75$ & 0,00 & 0,94 & 0,00 & $-2,52$ & 0,75 \\
\hline
\end{tabular}

Note: $200=$ scenario parallelo dei +200 punti base; $-200=$ scenario parallelo dei -200 punti base; $99^{\circ}=$ scenario del $99^{\circ}$ percentile; $1^{\circ}=$ scenario del $1^{\circ}$ percentile; SRU = scenario dello short rates shock up; SRD = scenario dello short rates shock down; STEEP = scenario steepener; FLAT = scenario flattener. Fonte: elaborazione esemplificativa dell'Autore a partire dai dati di bilancio di una singola banca al 31/12/2017.

\section{Conclusioni ed implicazioni di policy}

La determinazione del capitale interno a presidio del rischio di tasso di interesse del portafoglio bancario rappresenta un elemento di particolare criticità nell'ambito della stesura del Resoconto ICAAP. Ciò in quanto le evidenze sulla complessiva adeguatezza patrimoniale sono analizzate dalle Autorità di Vigilanza in sede SREP ai fini della determinazione 
per ciascuna banca degli Overall Capital Requirement (OCR) e della eventuale capital guidance. I livelli minimi degli indicatori di solvibilità da soddisfare, definiti dalle Autorità di vigilanza in sede SREP, influenzano la crescita dimensionale della banca. L'attività di erogazione del credito è, infatti, funzione della differenza tra il profilo di rischio della banca alle varie date di valutazione e i limiti imposti dalle Autorità di vigilanza in sede SREP.

Una maggiore esposizione sul rischio di tasso di interesse del portafoglio bancario derivante dalle innovazioni regolamentari oggetto di analisi nel presente contributo può, quindi, condurre ad un incremento dei requisiti aggiuntivi in sede SREP e, per tale via, ad una potenziale riduzione dell'offerta di credito all'economia e/o alla necessitaà di incrementare la propria dotazione patrimoniale. Quanto detto si inserisce, inoltre, in uno scenario finanziario di riferimento caratterizzato da basso livello dei tassi di interesse che, a seguito dell'applicazione del vincolo di non negatività, ha determinato degli effetti distorsivi dando luogo al fenomeno della neutralità al rischio. Ne consegue l'esigenza di avere una quanto più accurata e dettagliata comprensione delle determinanti dell'esposizione al rischio di tasso di interesse del portafoglio bancario nell'ambito dei due approcci di misurazione (valore economico e margine di interesse) e delle possibili modalità di integrazione delle evidenze ottenute al fine della quantificazione del capitale interno e/o di eventuali buffer di capitale a presidio del rischio in questione. Quanto detto facendo riferimento non solo agli scenari di variazione dei tassi di interesse attualmente in vigore ma anche a quelli proposti dal Comitato di Basilea nell'aprile 2016 e, successivamente, recepiti dalle linee guida dell'ABE dello scorso mese di luglio 2018.

Le evidenze empiriche derivanti dall'applicazione dei differenti scenari di variazione dei tassi di interesse nel periodo oggetto di analisi che va dal 2006 al 2017 ad un campione di 130 banche commerciali italiane confermano, come già sottolineato dalla precedente letteratura, la significativa presenza in un contesto di bassi livelli dei tassi di interesse del fenomeno della neutralità al rischio per le metodologie previste dall'attuale quadro normativo di vigilanza prudenziale. Il fenomeno della neutralità al rischio riguarda, con differenti specificità, sia l'approccio del valore economico sia quello del margine di interesse. Nell'ambito dell'approccio del valore economico si manifesta in maniera rilevante a partire dal 2012 a seguito del basso livello dei tassi di interesse vigente. L'introduzione dei nuovi scenari di variazione dei tassi di interesse, e nello specifico l'applicazione dello scenario steepener, riduce sensibilmente tale fenomeno nell'ambito dell'approccio del valore economico. Il numero di banche esposte a tale scenario in termini di valori medi sull'intero periodo oggetto di analisi risulta pari a 127,33. Nell'ambito dell'approccio del margine di interesse, invece, il fenomeno della neutralità al rischio assume una certa consistenza a partire dal 2015 quando si rilevano valori negativi nella parte a breve termine della struttura a termine dei tassi di interesse. La conseguente applicazione del vincolo di non negatività e, quindi, l'utilizzo di variazioni pari a zero fa sì che la maggior parte delle banche non siano esposte al rischio in questione a seguito dell'applicazione di tutti gli scenari di variazione in diminuzione dei tassi di interesse considerati nel corso della trattazione.

Sulla base di quanto sopra detto è evidente che un eventuale futuro aumento del livello della struttura per scadenza dei tassi di interesse facendo, progressivamente, venir meno il fenomeno della neutralità al rischio renderebbe esposte, con un relativo capitale interno da porre a presidio del rischio in questione, tutte quelle banche che attualmente risultano neutrali al rischio. Una situazione analoga potrebbe verificarsi anche a seguito della revisione del vincolo di non negatività, cosi come previsto nelle recenti linee guida dell'ABE, con la previsione di specifici floor negativi e crescenti sui vari nodi della struttura per scadenza dei tassi di interesse. Si tratta, quindi, di una questione di particolare criticità che le banche devono tener conto nella definizione della propria esposizione al rischio, sulla base delle ipotesi di sviluppo delle varie forme tecniche di impiego e di raccolta definite in sede di budget e di piano industriale.

L'approccio del valore economico e del margine di interesse impattano sul livello di solvibilità della banca mediante differenti modalità: il primo conduce ad un requisito di capitale interno da porre a presidio del rischio in questione; il secondo ha, invece, impatti di tipo reddituale determinando minori utili o maggiori perdite e, per tale via, contribuisce a determinare l'ammontare dei fondi propri e, quindi, la complessiva situazione di solvibilità della Banca.

L'introduzione degli impatti sul margine di interesse impone, tuttavia, la necessità di definire specifici criteri di integrazione delle evidenze ottenute dall'applicazione di uno specifico scenario di variazione dei tassi di interesse in entrambi gli approcci. Al riguardo le recenti linea guida $\mathrm{ABE}$ e la Circolare 285 non definiscono nel dettaglio le modalità di integrazione ma forniscono solo un insieme di utili principi a cui fare riferimento nell'ambito della valutazione dell'adeguatezza complessiva di capitale. L'analisi dell'attuale quadro normativo sembra, tuttavia, escludere eventuali ipotesi di aggregazione, o, in altri termini di impatto congiunto, in corrispondenza dei singoli scenari di variazione dei tassi di interesse ove si verifichi sia una riduzione del valore economico che del margine di interesse. Il presente contributo, al riguardo, propone un criterio di integrazione basato su un indicatore di solvibilità rettificato calcolato considerando sia i rischi di primo pilastro che quelli di secondo pilastro. Il criterio consente, nello specifico, di considerare gli impatti sul livello di solvibilità della banca in termini di capitale interno da porre a presidio del rischio in questione, nell'ambito dell'approccio del valore economico, e in termini di aumento/riduzione del livello dei fondi propri nell'ambito dell'approccio del margine di interesse. È auspicabile, al riguardo, la costituzione di appositi tavoli tecnici tra gli operatori del settore al fine di declinare, 
anche mediante un utile confronto con le Autorità di vigilanza, prassi comuni a cui fare riferimento, tenendo conto del principio di proporzionalità, nell'ambito della redazione del Resoconto ICAAP.

\section{Bibliografia}

Igor Gianfrancesco

- $\quad$ Abdymomunov A., Gerlach J., 2014. Stress testing interest rate risk exposure, in Journal of Banking and Finance 49, $287-301$.

- AIFIRM (2016), Risposta al documento di consultazione Interest rate risk in the banking book del Comitato di Basilea sulla vigilanza bancaria, in Newsletter AIFIRM, Anno 10, Numero 3. Il position paper è scaricabile anche dal sito del Comitato di Basilea al seguente link:

- $\quad$ http://www.bis.org/bcbs/publ/comments/d319/overview.htm

- Banca d'Italia (2005), Il bilancio bancario: schemi e regole di compilazione, Circolare n.262 del 22 dicembre 2005 e successivi aggiornamenti.

- $\quad$ Banca d'Italia (2006), Nuove disposizioni di vigilanza prudenziale per le banche, Circolare n.263 del 27 dicembre 2006 e successivi aggiornamenti.

- Banca d'Italia (2008), Matrice dei Conti, Circolare 272 del 30 luglio 2008 e successivi aggiornamenti.

- $\quad$ Banca d'Italia (2013), Disposizioni di vigilanza prudenziale per le banche, Circolare n.285 del 17 dicembre 2013 e successivi aggiornamenti.

- Basel Committee on Banking Supervision (2004), Principles for Management and Supervision of Interest Rate Risk. Bank for International Settlements.

- Basel Committee on Banking Supervision (2015), Interest rate risk in the banking book. Consultative Document. Bank for International Settlements, June.

- Basel Committee on Banking Supervision (2016), Interest rate risk in the banking book. Bank for International Settlements, June.

- Cocozza R, Curcio D., Gianfrancesco I. (2015), Non- Maturity Deposits and banks' exposure to the interest rate risk: issues arising from the Basel regulatory frame work, in Journal of Risk, 17(5), 1-36.

- Cerrone R., Cocozza R., Curcio D., Gianfrancesco I. (2017), Does prudential regulation contribute to effective measurement and management of interest rate risk? Evidence from Italian banks, in Journal of Financial Stability, 30, 126-138.

- Curcio D., Gianfrancesco I. (2011), La misurazione del rischio di tasso di interesse del portafoglio bancario nell'ambito di Basilea 2: quali le possibili criticità nella ricerca di nuove best practices? in Neswletter AIFIRM, Anno 6, Numero 1.

- Entrop, O., Memmel, C., Wilkens, M., and Zeisler, A. (2008), Analyzing the interest rate risk of banks using time series of accounting-based data: evidence from Germany, Deutsche Bundesbank Discussion Paper Series 2 No.1.

- $\quad$ Entrop, O., Wilkens, M., and Zeisler, A. (2009), Quantifying the Interest Rate Risk of Banks: Assumptions Do Matter, European Financial Management 15(5), 1001-1018.

- European Banking Authority (2015), Orientamenti sulla gestione del rischio di tasso di interesse derivante da attività diverse dalla negoziazione (non-trading activities), 10 ottobre.

- European Banking Authority (2014), Guidelines on ommon procedures and methodologies for the supervisory review and evaluation process (SREP), 19 December.

- $\quad$ European Banking Authority (2017), Question ID: 2017, 3121, Date of pubblication: 07/07/2017. Il documento è disponibile al seguente link: http://www.eba.europa.eu/single-rule-book-qa/-/qna/view/publicId/2017_3121

- $\quad$ European Banking Authority (2018), Guidelines on the management of interest rate risk arising from non-trading book activities. 19 July.

- $\quad$ Fiori, R., and Iannotti, S. (2007), Scenario Based Principal Component Value-at-Risk: An Application to Italian Bank's Interest Rate Risk Exposure, in Journal of Risk 9(3), 63-99.

- Gianfrancesco I., Giliberto C. (2014), La vischiosità dei depositi a vista durante la recente crisi finanziaria: implicazioni in una prospettiva di risk management, in Newsletter AIFIRM, Anno 9, Numero 3.

- Gianfrancesco I. (2016), L'esposizione al rischio di tasso di interesse del portafoglio bancario: quali implicazioni per le strategie di Asset \& Liability Management? in Newsletter AIFIRM, Anno 11, Numero 3-4.

- Gianfrancesco I. (2017), L'applicazione dei nuovi scenari di variazione dei tassi di interesse proposti dal Comitato di Basilea: quali implicazioni per le banche italiane? in Risk Management Magazine, Anno 12, Numero 3.

- Houpt, J. V., and Embersit, J. A. (1991), A Method for Evaluating Interest Rate Risk in U.S. Commercial Banks, in Federal Reserve Bulletin, 77(2), 625-637.

- $\quad$ Sierra, G. E. (2009), Can an accounting-based duration model effectively measure interest rate sensitivity? Il paper è disponibile sul sito SSRN al seguente link: https://papers.ssrn.com/sol3/papers.cfm?abstract_id=1488884

- $\quad$ Sierra G. E., and Yeager T. J. (2004), What Does the Federal Reserve's Economic Value Model Tell Us About Interest Rate Risk at U.S. Community Banks? in The Federal Reserve Bank of St. Louis Review 86(6).

- Wright D. M., and Houpt J. V. (1996), An Analysis of Commercial Bank Exposure to Interest Rate Risk, in Federal Reserve Bulletin 82(2), 115-28. 


\section{Confronto tra l'approccio tradizionale e le tecniche di Machine Learning per la model- lizzazione della stagionalità nella valorizzazione degli swap indicizzati all'inflazione}

di $^{1}$ Ottavio Caligaris (Università di Genova) - Pier Giuseppe Giribone (Banca Carige e Università di Genova)

\section{Abstract}

Un Inflation-Indexed Swap (IIS) è un derivato nel quale, a ciascuna data di pagamento, una controparte scambia un tasso inflattivo contro un tasso fisso. Nella determinazione dei cash flow associati alla gamba inflattiva dello swap occorre predisporre un modello matematico atto alla proiezione dei valori dell'indice inflattivo CPI nel futuro. Al fine di ricavare tali simulazioni, si ricorre nella normale prassi di valutazione, ad un modello di mercato che si basa tipicamente sulle quotazioni degli zero-coupon IIS. Tale informazione è utile al fine di ricavare il trend principale del valore atteso prospettico della CPI, pur rimanendo insufficiente nella adeguata descrizione dell'indice inflattivo. Questo modello deve essere integrato con un altro, il cui compito è quello di descrivere il caratteristico effetto di stagionalità della CPI. Gli autori propongono l'applicazione di un modello di stagionalità basato su reti neurali feed-forward con neuroni circolari, come noto, tecnica di Machine Learning particolarmente idonea nell'individuazione della periodicità in una serie storica. Il paper può essere suddiviso in quattro sezioni: nella prima viene descritta la meccanica di funzionamento e le metodologie di pricing per i due swap indicizzati all'inflazione più popolari: lo zero-coupon (ZCIIS) swap e il year-on-year (YYIIS) swap. Nella seconda parte viene descritto il modello tradizionale base impiegato per ricavare i valori prospettici (trend + stagionalità) dell'indice inflattivo. Nella terza parte dello studio viene presentata l'architettura, il funzionamento e la validazione della rete neurale impiegata per descrivere l'effetto periodico dell'indice. L'ultima sezione confronta le due tecniche e valuta l'impatto del modello nella valorizzazione a parametri di mercato di un Year-on-Year swap indicizzato all'inflazione europea.

An Inflation-Indexed Swap (IIS) is a derivative in which, at every payment date, a counterparty swaps an inflation rate with a fixed rate. For the calculation of the Inflation Leg cash flows it is necessary to build a mathematical model suitable for the CPI projection. For this purpose, analysts usually start to use market quotes for the Zero-Coupon swaps in order to derive the future trend of the inflation index, together with a seasonality model for capturing the typical periodical effects. Authors propose a forecasting model for inflation seasonality based on feed-forward artificial neural networks with circular neurons: a specific Machine Learning technique suitable for identifying the cyclic nature of time series. The paper is structured in four sections: section 1 illustrates the pricing methodologies for the two most popular IIS: the zero Coupon Inflation-Indexed Swap (ZCIIS) and the Year-on-Year Inflation-Indexed Swap (YYIIS); section 2 deals with the traditional base method for the forecast of CPI values (trend + seasonality); section 3 describes the architecture, the working principle and the validation of the neural network; section 4 concludes comparing the impact of the two seasonality models on the fair-value of an inflationindexed Year-on-Year swap.

\section{Key Words:}

Inflation-Indexed Swap (IIS), Year-on-Year Inflation-Indexed Swap (YYIIS), Zero-Coupon Inflation-Indexed Swap (ZCIIS), Seasonality model, CPI bootstrap, Machine Learning (ML), Artificial Neural Network (ANN), Feed-Forward Neural Network, Circular Neuron

\section{Introduzione}

Le metodologie di Machine Learning si stanno sempre maggiormente diffondendo nell'ambito finance.

Tra i numerosi esempi di applicazioni proposte dalla letteratura si riscontrano che quelle più popolari sono rivolte principalmente alla risoluzione dei problemi seguenti:

- valutazione della bontà dei dati di input (data quality) [17]

- tecniche innovative di algo-trading [8]

- gestione ottimale di un portafoglio [10], [20]

- classificazione e riconoscimento di particolari pattern [13]

- predizione di valori futuri di serie storiche dei prezzi, in alternativa agli approcci econometrici tradizionali [15], [19]

Più difficile trovare un riscontro nella letteratura di applicazioni delle metodologie di intelligenza artificiale legati alla determinazione del fair-value di strumenti finanziari complessi.

L'impiego tradizionale classico che si effettua è quello di risolvere numericamente la PDE fondamentale di pricing di BlackScholes-Merton impiegando funzioni a base radiale [7].

Il paper proposto mira ad aumentare la letteratura esistente in materia di pricing di derivati esotici, proponendo la costruzione di una particolare rete multi-layer feed-forward dotata di neuroni circolari in applicazione a contratti swap indicizzati all'indice inflattivo. Tale modellizzazione riveste un carattere di innovazione e non si sono riscontrati studi si tale tematica nella recente letteratura.

${ }^{1}$ Articolo sottoposto a doppio referaggio anonimo, pervenuto il 11/9/2018 e accettato il 31/10/2018. 


\section{Funzionamento e valutazione degli inflation-indexed swap}

Un Inflation-Indexed Swap (IIS) è un contratto swap dove, a ciascuna data di pagamento, $T_{1}, \ldots, T_{M}$, la controparte A paga alla controparte B il tasso inflattivo su un periodo predefinito, mentre la controparte B paga alla controparte A un tasso fisso. Il tasso inflattivo è calcolato come il ritorno percentuale dell'indice CPI lungo il periodo temporale di riferimento. Due sono le principali tipologie di IIS negoziati sul mercato: lo Zero-Coupon Inflation-Indexed Swap (ZCIIS) e il Year-on-Year InflationIndexed Swap (YYIIS) [16]. In uno ZCIIS, al tempo finale $T_{M}$, assumendo $T_{M}=M$ anni, la controparte B paga alla controparte A il quantitativo fisso:

$$
N\left[(1+K)^{M}-1\right](1)
$$

Dove $K$ e $N$ sono rispettivamente il tasso fisso contrattualizzato e il valore nominale.

In cambio, per questo pagamento fisso, la controparte A paga alla controparte B alla scadenza $T_{M}$, l'ammontare variabile:

$$
N\left[\frac{I\left(T_{M}\right)}{I_{0}}-1\right]
$$

In un YYIIS, a ciascun tempo $T_{i}$, la controparte B paga alla controparte A l'ammontare fisso:

$$
N \varphi_{i} K(3)
$$

Dove $\varphi_{i}$ è la frazione d'anno della fixed-leg nell'intervallo $\left[T_{i-1}, T_{i}\right], T_{0}:=0$ e $N$ il valore nominale del contratto.

La controparte A paga alla controparte B il quantitativo variabile:

$$
N \varphi_{i}\left[\frac{I\left(T_{i}\right)}{I\left(T_{i-1}\right)}-1\right]
$$

Dove $\varphi_{i}$ è la frazione d'anno della floating-leg nell'intervallo $\left[T_{i-1}, T_{i}\right], T_{0}:=0$ e $N$ è sempre il valore nominale del contratto. Gli ZCIIS e gli YYIIS sono quotati dal mercato in termini del corrispondente tasso fisso equivalente $K$ : nella Tabella 1 sono riportati i valori dei tassi al 29 Giugno 2018. La CPI di riferimento impiegata è l'indice dell'Euro-zona ex-tobacco (identificativo Bloomberg®: CPTFEMU Index).

\begin{tabular}{|c|c|c|c|c|c|c|}
\hline Tenor & ZC Bid & ZC Mid & ZC Ask & YoY Bid & YoY Mid & YoY Ask \\
\hline 1 YR & 1,670 & 1,680 & 1,690 & 1,670 & 1,680 & 1,690 \\
\hline 2 YR & 1,530 & 1,555 & 1,580 & 1,530 & 1,555 & 1,580 \\
\hline 3 YR & 1,486 & 1,526 & 1,566 & 1,486 & 1,526 & 1,566 \\
\hline 4 YR & 1,500 & 1,525 & 1,550 & 1,500 & 1,525 & 1,550 \\
\hline 5 YR & 1,513 & 1,528 & 1,543 & 1,512 & 1,528 & 1,543 \\
\hline 6 YR & 1,495 & 1,545 & 1,595 & 1,495 & 1,545 & 1,594 \\
\hline 7 YR & 1,508 & 1,558 & 1,608 & 1,507 & 1,557 & 1,607 \\
\hline 8 YR & 1,559 & 1,579 & 1,599 & 1,557 & 1,578 & 1,598 \\
\hline 9 YR & 1,582 & 1,602 & 1,622 & 1,579 & 1,600 & 1,621 \\
\hline 10 YR & 1,610 & 1,625 & 1,640 & 1,606 & 1,622 & 1,638 \\
\hline 12 YR & 1,663 & 1,683 & 1,703 & 1,656 & 1,676 & 1,696 \\
\hline 15 YR & 1,703 & 1,753 & 1,803 & 1,693 & 1,740 & 1,788 \\
\hline 20 YR & 1,834 & 1,859 & 1,884 & 1,810 & 1,836 & 1,862 \\
\hline 25 YR & 1,918 & 1,933 & 1,948 & 1,884 & 1,901 & 1,919 \\
\hline 30 YR & 1,968 & 1,993 & 2,018 & 1,928 & 1,953 & 1,978 \\
\hline
\end{tabular}

Tabella 1. Euro Inflation Swap Rate - 29 Giugno 2018 - Fonte: Bloomberg ${ }^{\circledR}$

\subsection{La valorizzazione di un ZCIIS}

La teoria standard di valorizzazione in assenza di arbitraggio porta alla determinazione del fair value della gamba inflattiva di uno ZCIIS al tempo $t, 0 \leq t \leq T_{M}[2]$ : 


$$
\operatorname{ZCIIS}\left(t, T_{M}, I_{0}, N\right)=N \cdot E_{n}\left\{\exp \left(-\int_{t}^{T_{M}} n(u) d u\right)\left[\frac{I\left(T_{M}\right)}{I_{0}}-1\right] \mid \mathrm{F}_{t}\right\}
$$

Dove $\mathrm{F}_{t}$ denota la $\sigma$-algebra generata dal processo stocastico del sottostante sino al tempo $t$.

Il prezzo nominale di uno zero coupon bond eguaglia il prezzo nominale di un contratto, che paga un'unità dell'indice CPI alla scadenza del titolo. In formule, per ciascun $t<T$ :

$$
I(t) P_{r}(t, T)=I(t) E_{r}\left\{\exp \left(-\int_{t}^{T} r(u) d u\right) \mid \mathrm{F}_{t}\right\}=E_{n}\left\{\exp \left(-\int_{t}^{T} n(u) d u\right) I(T) \mid \mathrm{F}_{t}\right\}(6)
$$

Quindi la (5) diventa:

$$
\operatorname{ZCIIS}\left(t, T_{M}, I_{0}, N\right)=N\left[\frac{I(t)}{I_{0}} P_{r}\left(t, T_{M}\right)-P_{n}\left(t, T_{M}\right)\right]
$$

La (7) per una valutazione al tempo $t=0$ si semplifica:

$$
\operatorname{ZCIIS}\left(0, T_{M}, N\right)=N\left[P_{r}\left(0, T_{M}\right)-P_{n}\left(0, T_{M}\right)\right]
$$

Le formule (7) e (8) portano ad avere una valutazione del derivato indipendente dalle assunzioni fatte dal modello impiegato per descrivere l'evoluzione del mercato dei tassi d'interesse e che derivano semplicemente da ragionamenti in assenza di arbitraggio. Questo risultato è estremamente importante, dal momento che consente di derivare senza ambiguità i prezzi degli zero-coupon bond a partire dai prezzi quotati degli zero-coupon inflation-indexed swap [2].

Infatti, eguagliando la (8) con il valore nominale attualizzato della (1) e ricavando $P_{n}\left(0, T_{M}\right)$ dalla curva corrente degli zerocoupon nominali, si è in grado di risolvere l'equazione per la quantità incognita $P_{r}\left(0, T_{M}\right)$.

Si ottiene pertanto:

$$
P_{r}\left(0, T_{M}\right)=P_{n}\left(0, T_{M}\right)\left[1+K\left(T_{M}\right)\right]^{M}(9)
$$

I $P_{r}\left(0, T_{M}\right)$ per le scadenze intermedie possono essere inferenziati tenendo conto degli effetti tipici di stagionalità dell'inflazione.

Kazziha [12] ha definito la $T$-forward CPI al tempo $t$ come il quantitativo fisso $X$ da scambiare al tempo $T$ per la CPI $I(T)$, per il quale un tale swap ha un valore nullo.

Dalla formula (6), si ottiene:

$$
I(t) P_{r}(t, T)=X P_{n}(t, T)(10)
$$

Il valore al tempo zero di una $T_{M}$-forward CPI può essere ottenuta dalla quotazione di mercato $K\left(T_{M}\right)$ applicando la formula:

$$
I_{M}(0)=I(0) \cdot\left[1+K\left(T_{M}\right)\right]^{M}
$$

Tale risultato è perfettamente equivalente alla $(9)$.

\subsection{La valorizzazione di un YYIIS}

La valutazione di uno YYIIS, rispetto a quella di uno ZCIIS risulta più complicata: infatti il valore al tempo $t<T_{i}$ del pay-off (4) al tempo $T_{i}$ è [2]:

$$
Y Y I I S\left(t, T_{i-1}, T_{i}, \psi_{i}, N\right)=N \psi_{i} E_{n}\left\{\exp \left(-\int_{t}^{T_{i}} n(u) d u\right)\left[\frac{I\left(T_{i}\right)}{I\left(T_{i-1}\right)}-1\right] \mid \mathrm{F}_{t}\right\}
$$

Assumendo $t<T_{i-1}$ può essere calcolata come:

$$
N \psi_{i} E_{n}\left\{\exp \left(-\int_{t}^{T_{i-1}} n(u) d u\right) E_{n}\left\{\exp \left(-\int_{T_{i-1}}^{T_{i}} n(u) d u\right)\left[\frac{I\left(T_{i}\right)}{I\left(T_{i-1}\right)}-1\right] \mid \mathrm{F}_{t-1}\right\} \mid \mathrm{F}_{t}\right\}
$$

Il valore atteso più interno all'equazione (13) è lo $Z C I I S\left(T_{i-1}, T_{i}, I\left(T_{i-1}\right), 1\right)$ : 


$$
\begin{aligned}
& N \psi_{i} E_{n}\left\{\exp \left(-\int_{t}^{T_{i-1}} n(u) d u\right)\left[P_{r}\left(T_{i-1}, T_{i}\right)-P_{n}\left(T_{i-1}, T_{i}\right)\right] \mid \mathrm{F}_{t}\right\}= \\
& =N \psi_{i} E_{n}\left\{\exp \left(-\int_{t}^{T_{i-1}} n(u) d u\right)\left[P_{r}\left(T_{i-1}, T_{i}\right)\right] \mid \mathrm{F}_{t}\right\}-N \psi_{i} P_{n}\left(t, T_{i}\right)
\end{aligned}
$$

L'ultimo valore atteso può essere visto come il prezzo nominale di un derivato che paga in unità nominali il prezzo dello zerocoupon bond, $P_{r}\left(T_{i-1}, T_{i}\right)$ al tempo $T_{i-1}$.

Se i tassi reali fossero deterministici, allora questo prezzo sarebbe il valore scontato, in termini nominali, del prezzo forward di un bond [2]. In questo caso si avrebbe:

$$
E_{n}\left\{\exp \left(-\int_{t}^{T_{i-1}} n(u) d u\right)\left[P_{r}\left(T_{i-1}, T_{i}\right)\right] \mid \mathrm{F}_{t}\right\}=P_{r}\left(T_{i-1}, T_{i}\right) P_{n}\left(t, T_{i-1}\right)=\frac{P_{r}\left(t, T_{i}\right)}{P_{r}\left(t, T_{i-1}\right)} P_{n}\left(t, T_{i-1}\right)
$$

In pratica, comunque, i tassi reali sono stocastici ed il valore atteso nella (14) è dipendente dal modello. In questa sezione ci si ripropone la valorizzazione del YYIIS con il modello di Jarrow-Yildirim (JY model) [11].

Denotando con $Q_{n}^{T}$ la misura nominale T-forward per una generale scadenza $T$ e con $E_{n}^{T}$ il valore atteso associato, si può scrivere [2]:

$$
Y Y I I S\left(t, T_{i-1}, T_{i}, \psi_{i}, N\right)=N \psi_{i} P_{n}\left(t, T_{i-1}\right) E_{n}^{T_{i-1}}\left\{P_{r}\left(T_{i-1}, T_{i}\right) \mid \mathrm{F}_{t}\right\}-N \psi_{i} P_{n}\left(t, T_{i}\right)
$$

Ricordando la formula per uno zero-coupon bond price in accordo con il modello di Hull-White [9]:

$$
P_{r}(t, T)=A_{r}(t, T) \exp \left[-B_{r}(t, T) \cdot r(t)\right]
$$

Dove:

$B_{r}(t, T)=\frac{1}{a_{r}}\left[1-\exp \left[-a_{r}(T-t)\right]\right]$

$A_{r}(t, T)=\frac{P_{r}^{M}(0, T)}{P_{r}^{M}(0, t)} \exp \left\{B_{r}(t, T) f_{r}^{M}(0, t)-\frac{\sigma_{r}^{2}}{4 a_{r}}\left[1-\exp \left(-2 a_{r} t\right)\right] B_{r}(t, T)^{2}\right\}$

E considerando che il tasso reale instantaneo evolve sotto $Q_{n}^{T_{i}-1}$, in accordo con l'equazione differenziale stocastica:

$$
d r(t)=\left[-\rho_{n, r} \sigma_{n} \sigma_{r} B_{n}\left(t, T_{i-1}\right)+\vartheta_{r}(t)-\rho_{r, I} \sigma_{I} \sigma_{r}-a_{r} r(t)\right] d t+\sigma_{r} d W_{r}^{T i-1}(t)
$$

Con $W_{r}^{T_{i-1}}$ un moto browniano sotto la misura $Q_{n}^{T_{i}-1}$, si ottiene:

$$
Y Y I I S\left(t, T_{i-1}, T_{i}, \psi_{i}, N\right)=N \psi_{i} P_{n}\left(t, T_{i-1}\right) \frac{P_{r}\left(t, T_{i}\right)}{P_{r}\left(t, T_{i-1}\right)} \exp \left[C\left(t, T_{i-1}, T_{i}\right)\right]-N \psi_{i} P_{n}\left(t, T_{i}\right)
$$

Dove:

$C\left(t, T_{i-1}, T_{i}\right)=\sigma_{r} B_{r}\left(T_{i-1}, T_{i}\right)\left[B_{r}\left(t, T_{i-1}\right)\left(\rho_{r, I} \sigma_{I}-\frac{1}{2} \sigma_{r} B_{r}\left(t, T_{i-1}\right)+\frac{\rho_{n, r} \sigma_{n}}{a_{n}+a_{r}}\left(1+a_{r} B_{n}\left(t, T_{i-1}\right)\right)\right)-\frac{\rho_{n, r} \sigma_{n}}{a_{n}+a_{r}} B_{n}\left(t, T_{i-1}\right)\right]$

Il valore atteso del prezzo di uno zero-coupon bond sotto la misura nominale forward, nel modello di Yarrow-Yildirim, è quindi pari al prezzo forward corrente del bond moltiplicato per un fattore correttivo, che dipende dalle volatilità instantanee del tasso nominale $\sigma_{n}$, del tasso reale $\sigma_{r}$, della CPI $\sigma_{I}$ e dalla correlazione instantanea tra il tasso reale e la CPI $\rho_{r, I}$ [2].

L'esponenziale di $C$ rappresenta il termine correttivo sopra menzionato: questo tiene conto della stocasticità dei tassi reali e, pertanto, svanisce per $\sigma_{r}=0$.

Il valore al tempo $t$ della gamba indicizzata all'inflazione dello swap è ottenuta sommando tutti i pagamenti floating. Pertanto:

$$
\begin{aligned}
& Y Y I I S(t, \mathcal{T}, \Psi, N)=N \psi_{\iota(t)}\left[\frac{I(t)}{I\left(T_{\iota(t)-1}\right)} P_{r}\left(t, T_{\iota(t)}\right)-P_{n}\left(t, T_{\iota(t)}\right)\right]+ \\
& N \sum_{i=\iota(t)+1}^{M} \psi_{i}\left[P_{n}\left(t, T_{i-1}\right) \frac{P_{r}\left(t, T_{i}\right)}{P_{r}\left(t, T_{i-1}\right)} \exp \left[C\left(t, T_{i-1}, T_{i}\right)\right]-P_{n}\left(t, T_{i}\right)\right]
\end{aligned}
$$


Dove: $\mathcal{T}:=\left\{T_{1}, \ldots, T_{M}\right\}, \Psi:=\left\{\psi_{1}, \ldots, \psi_{M}\right\}$ e $\iota(t)=\min \left\{i: T_{i}>t\right\}$ con il primo pagamento dopo il tempo $t$ pagato in accordo $\operatorname{con}(7)$.

In particolare per $t=0$ :

$$
Y Y I I S(0, \mathcal{T}, \Psi, N)=N \psi_{1}\left[P_{r}\left(0, T_{1}\right)-P_{n}\left(0, T_{1}\right)\right]+N \sum_{i=2}^{M} \psi_{i}\left[P_{n}\left(0, T_{i-1}\right) \frac{P_{r}\left(0, T_{i}\right)}{P_{r}\left(0, T_{i-1}\right)} \exp \left[C\left(0, T_{i-1}, T_{i}\right)\right]-P_{n}\left(0, T_{i}\right)\right]
$$

\section{La simulazione tradizionale dei valori prospettici dell'indice CPI}

I valori forward della CPI, $\mathfrak{J}_{i}$ sono stimati a partire dagli Zero Swap Rate sull'inflazione applicando la formula (11), di seguito nuovamente riproposta:

$$
\mathfrak{I}_{M}(0)=\mathfrak{I}_{R E F}(0) \cdot\left[1+K\left(T_{M}\right)\right]^{M}
$$

Dove:

$\mathfrak{I}_{R E F}(0)$ è il valore CPI di riferimento. Esso corrisponde a quello fissato in corrispondenza a $n$ mesi indietro, rispetto alla data di riferimento per il bootstrap. Tipicamente si assume il lag temporale standard pari a 3 mesi e $K\left(T_{M}\right)$ e l'Inflation Zero Swap Rate quotato dal mercato in corrispondenza dell'anno $T_{M}$.

Con la relazione (11) viene simulato il valore futuro dell'indice in corrispondenza del tasso Swap rilevato dal mercato. Dato che la frequenza con cui viene pubblicato l'indice è mensile, è necessario fornire una simulazione della CPI con tale periodicità [18].

I punti della curva mancanti vengono pertanto stimati aggiungendo tra un valore calcolato $\mathfrak{J}_{M}(0)$ ed il suo successivo $\mathfrak{I}_{M+1}(0)$ il logaritmo dell'incremento mensile:

$$
\Delta \widetilde{I}_{M}=\frac{\ln \left(\frac{\mathfrak{\Im}_{M+1}(0)}{\mathfrak{I}_{M}(0)}\right)}{12 \cdot \tau}
$$

Dove $\tau$ è l'intervallo temporale espresso in anni compreso tra $\mathfrak{I}_{M}(0)$ e $\mathfrak{I}_{M+1}(0)$.

I punti costituenti la curva simulata dell'indice dei prezzi al consumo sono definiti dalla formula:

$$
\mathfrak{I}_{i+1}=\mathfrak{I}_{i} \cdot \exp \left(\Delta \mathfrak{I}_{M}+\mathfrak{R}_{M}\right) \operatorname{per} \mathfrak{I}_{M}(0) \leq \mathfrak{I}_{i} \leq \mathfrak{I}_{M+1}(0)
$$

Il metodo base di valorizzazione prevede di tenere conto della stagionalità dell'indice, sommando algebricamente i residui normalizzati $\mathfrak{R}_{M}$ ricavati dai valori storici della CPI, in accordo con l'espressione (27):

$$
\mathfrak{R}_{M}=\frac{\sum_{i=1}^{\text {stagyear }} \ln \left[\frac{\mathfrak{s}_{i+1}^{\text {Monthly }}}{\mathfrak{\Im}_{i}^{\text {Monthly }}}\right]}{\text { stagyear }}-\frac{\sum_{i=1}^{12 \cdot \text { stagyear }} \ln \left[\frac{\mathfrak{s}_{i+1}^{\text {Monthly }}}{\mathfrak{s}_{i}^{\text {Monthly }}}\right]}{12 \cdot \text { stagyear }}
$$

Dove:

$\mathfrak{R}_{M}$ sono i residui normalizzati ricavati dall'effetto della stagionalità su stagyear anni.

Il primo contributo è la variazione logaritmica dei valori CPI del mese considerato; il secondo rappresenta la variazione logaritmica complessiva registrata nel periodo temporale considerata per la stagionalità [18].

Ad esempio per calcolare il residuo normalizzato da associare al mese di settembre $\left(\mathfrak{R}_{9}\right)$ prendendo come campione di riferimento 5 anni (standard di mercato), stagyear $=5$, il primo contributo sarà calcolato dalla media delle variazioni logaritmiche registrate nei soli mesi di settembre.

Il secondo termine invece costituisce la grande media, calcolata nell'intero arco temporale di riferimento (60 mesi):

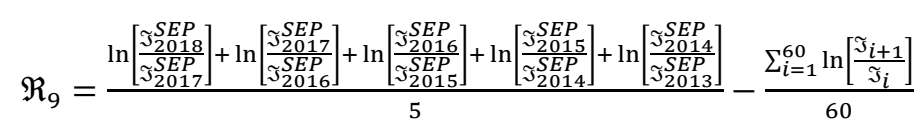




\section{La simulazione della stagionalità mediante reti neurali feed-forward con neuroni circolari}

Come noto gli elementi costitutivi di una rete neurale sono i singoli neuroni e le connessioni che si stabiliscono tra gli stessi. Un neurone si può schematizzare come una unità che riceve in ingresso un certo numero di segnali, li somma, li processa mediante una funzione di attivazione e restituisce un segnale in uscita [3]. La funzione di attivazione $s$ assume diverse forme a seconda del ruolo che il neurone, e la rete neurale cui è collegato, sono destinati a svolgere. In particolare, possiamo scegliere di usare come funzione di attivazione la funzione identica o, più in generale, una funzione lineare. In questo caso si assume che il neurone è lineare. Schematicamente può essere rappresentato come nella Figura 1, a seconda che la funzione di attivazione sia non lineare (Fig. 2, sinistra) o lineare (Fig. 2, destra).
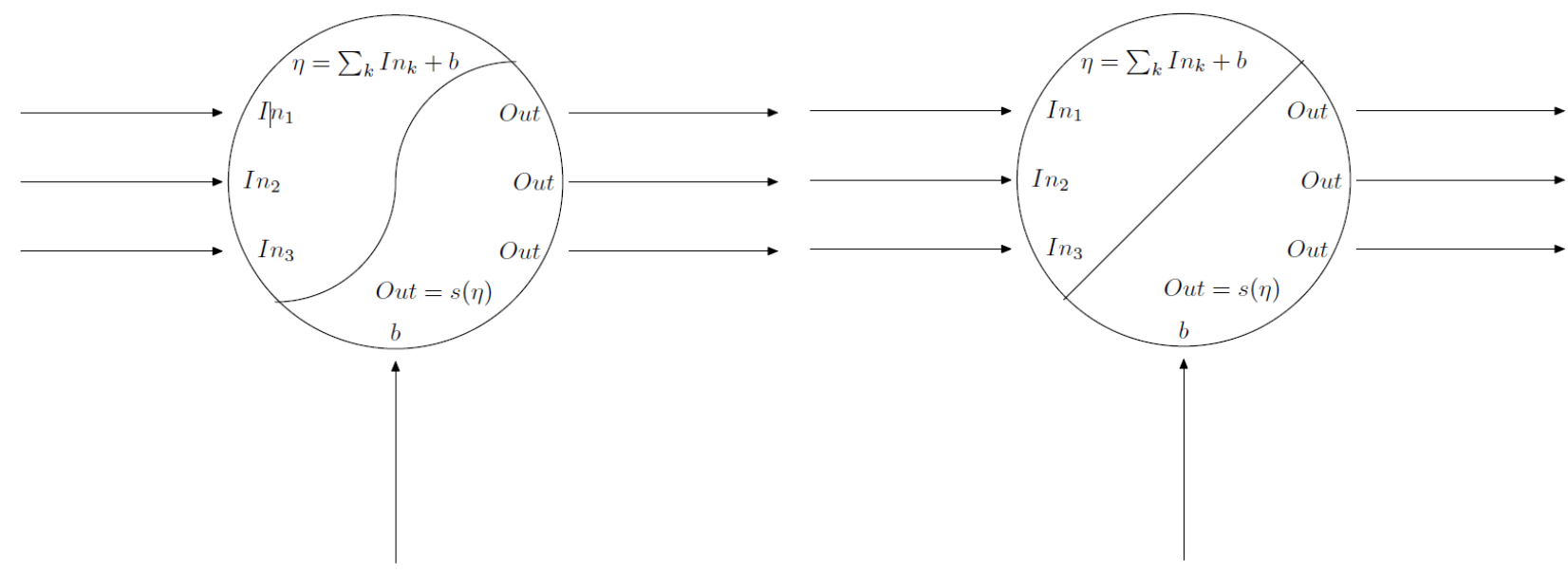

Figura 1. Neurone non lineare (a sinistra) e Neurone lineare (a destra)

Le funzioni di attivazione $s$ più diffuse sono:

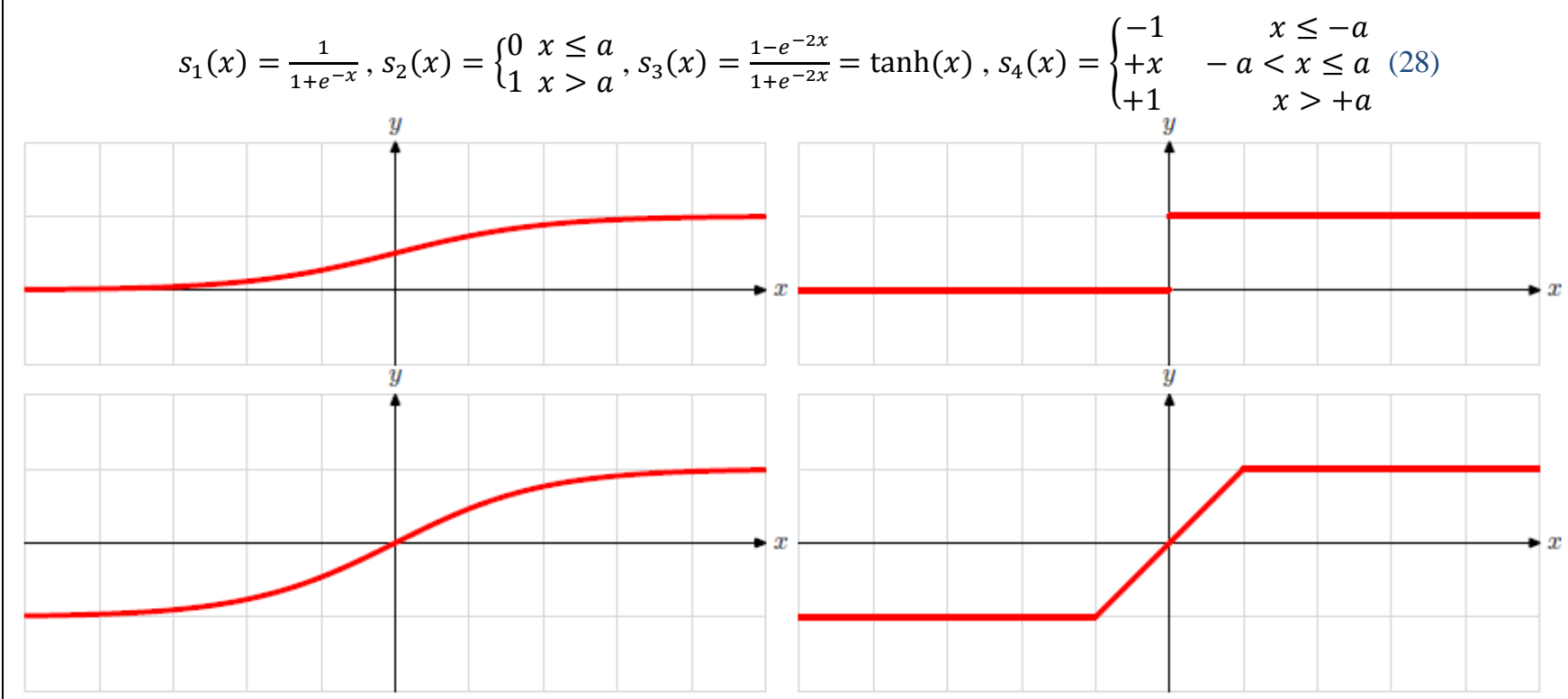

Figura 2. Le più comuni funzioni di attivazione: $s_{1}$ la Sigmoide, $s_{2}$ il Gradino, $s_{3}$ la Tangente iperbolica, $s_{4}$ la Rampa

Dal momento che per ottimizzare la rete occorre calcolare la derivata di $s$, è vantaggioso utilizzare la sigmoide $s_{1}=\sigma(x)=$ $\frac{1}{1+e^{-x}}$ oppure la tangente iperbolica $s_{3}=\tau(x)=\tanh (x)$, in quanto si verifica subito che $\sigma^{\prime}(x)=\sigma(1-\sigma)$ e $\tau^{\prime}(x)=1-$ $\tau^{2}=[1-\tau(x)][1+\tau(x)]$. Tale scelta semplifica in maniera significativa l'algoritmo di back-propagation, mediante il quale la rete apprende dall'insieme di dati, il training set, che le vengono forniti.

Una rete neurale è inoltre caratterizzata da strati di neuroni tra loro interconnessi; ogni neurone appartenente ad uno strato riceve segnali da tutti i neuroni dello strato precedente e invia la propria uscita a tutti i neuroni dello strato successivo [5]. Nella Figura 3 sono riportati a titolo esemplificativo i collegamenti tra tre strati successivi. 


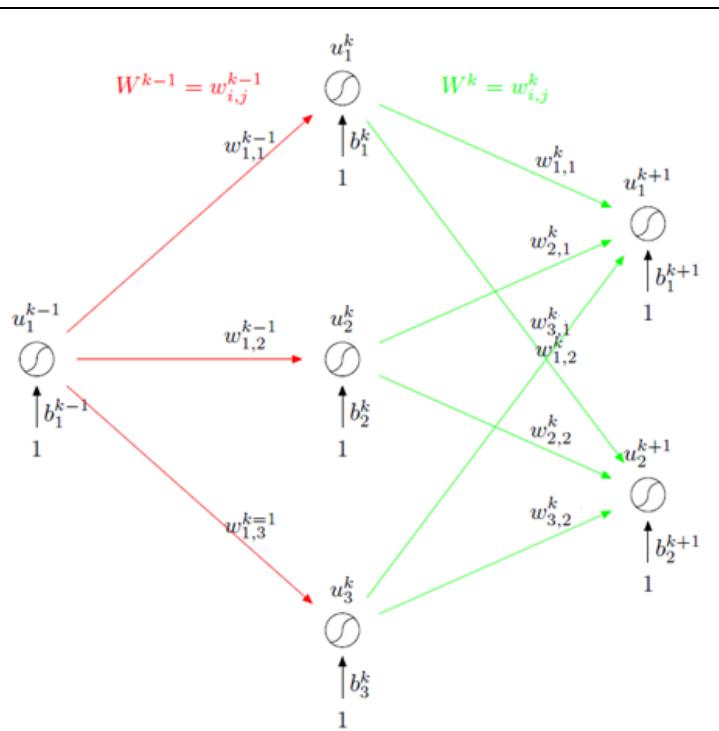

Figura 3. I collegamenti tra tre strati successivi della rete neurale

Nella Figura 4 sono evidenziati i collegamenti del $k$-esimo neurone dello strato $i$-esimo, a seconda che la funzione di attivazione sia non lineare o lineare.

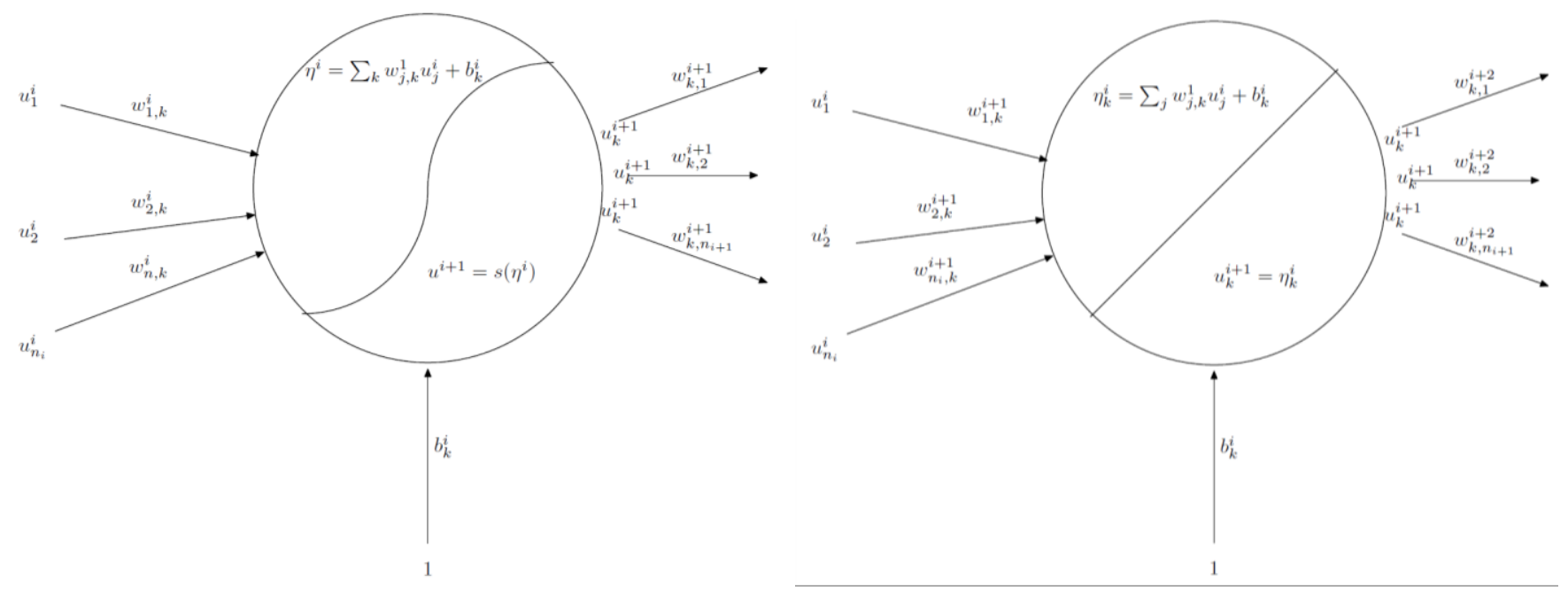

Figura 4. I collegamenti del $k$-esimo neurone dello strato i-esimo nel caso non lineare (sinistra) e lineare (destra)

Nella Figura 5 sono rappresentati il $k$-esimo neurone del primo e dell'ultimo strato.
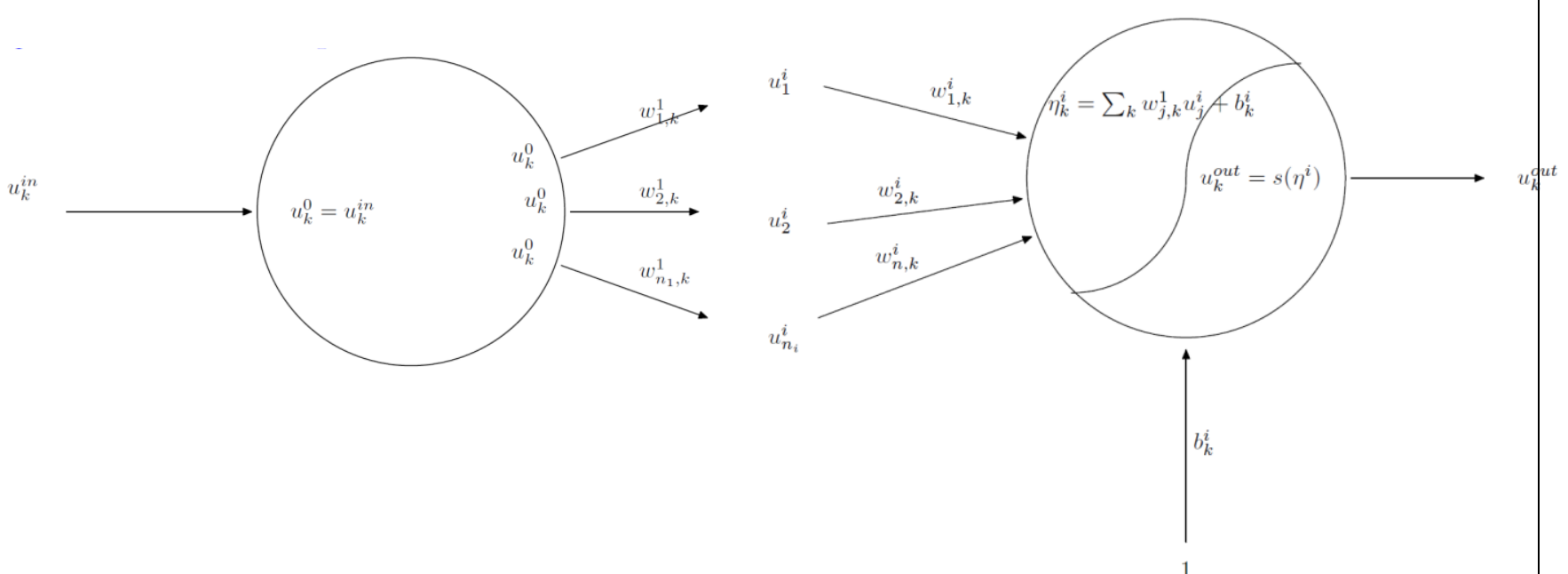

Figura 5. Il k-esimo neurone del primo e dell'ultimo strato 
I collegamenti trasferiscono l'uscita dei neuroni dello strato precedente ai neuroni di quello successivo, pesandola con coefficienti che saranno modificati durante il processo di apprendimento attuato durante l'ottimizzazione della rete [4]. Più precisamente, se lo strato $i$-esimo è composto da $n_{i}$ neuroni, il suo output definisce un vettore riga: $\left(u_{1}^{i}, \ldots, u_{n_{i}}^{i}\right)=u^{i}$. Sia $W^{i}=\left\{w_{j, k}^{i}\right\}$ la matrice dei pesi assegnati ai collegamenti tra gli strati $i$ ed $i+1$ e sia $b^{i}=\left(b_{1}^{i}, b_{2}^{i}, \ldots, b_{n_{i}}^{i}\right)$ il vettore dei coefficienti che pesano l'ingresso costante, posto ad 1, di ciascun neurone, solitamente indicato come bias: $\bar{W}^{i}=\left(\begin{array}{c}W^{i} \\ b^{i}\end{array}\right)$. Allora il vettore riga $u^{i+1}=\left(u_{1}^{i+1}, \ldots, u_{n+1}^{i+1}\right)$ si ottiene mediante la $u^{i+1}=\sigma\left(u^{i} \overline{W^{\imath}}\right)=\sigma\left(u^{i} W^{i}+b^{i}\right)$.

Il numero di neuroni del primo strato definisce il numero delle variabili in ingresso, mentre le variabili in uscita sono tante quante i neuroni dell'ultimo strato. Pertanto se, ad esempio, il primo strato conta $n$ neuroni e l'ultimo è costituito da $m$ neuroni, la rete neurale definisce una funzione da $\mathbb{R}^{n} \rightarrow \mathbb{R}^{m}$ e se i neuroni sono lineari, ne risulta anche lineare la funzione.

Può anche essere comodo aggiungere alla rappresentazione della rete un ulteriore strato, che fornisce una stima dello scostamento dell'uscita della rete $u_{O U T}$ (che corrisponde all'ingresso $u_{I N}$ ) dal valore atteso $u_{\text {TARGET }}$.

Normalmente lo scostamento è misurato da $\frac{1}{2}\left\|u_{\text {OUT }}-u_{\text {TARGET }}\right\|^{2}$.

E' interessante notare che se si pone come $s$ la funzione identità, e quindi si utilizzano neuroni lineari, si possono rappresentare facilmente ed efficacemente strutture algebriche lineari. Una rete neurale con neuroni lineari viene rappresentata come illustrato nella Figura 6. La prima delle due reti definisce l'applicazione lineare, che in termini matriciali si può scrivere $\left(x_{1}, x_{2}, x_{3}\right) W^{1} W^{2} W^{3}=\left(y_{1}, y_{2}\right)$ essendo il prodotto matriciale inteso colonne per righe, mentre l'uso di funzioni di attivazione non lineari consente ulteriore flessibilità.

La rete neurale rappresentata nella Figura 6 può essere utilizzata per rappresentare una funzione da $\mathbb{R}^{3}$ a $\mathbb{R}^{2}$. Oltre che dalla sua geometria, la rete è identificata dalle matrici: $W^{1}=\left(w_{j, k}^{1}\right), W^{2}=\left(w_{j, k}^{2}\right)$ e $W^{3}=\left(w_{j, k}^{3}\right)$ le cui dimensioni sono rispettivamente $3 \times 4,4 \times 3$ e $3 \times 2$ e dai vettori bias $b^{1}=\left(b_{k}^{1}\right), b^{2}=\left(b_{k}^{2}\right), b^{3}=\left(b_{k}^{3}\right)$ che hanno rispettivamente 4,3 e 2 componenti.

Siano $u^{0}, u^{1}, u^{2}, u^{3}$ i vettori che descrivono le uscite di ciascuno strato e che sono composti, a loro volta di $3,4,3$ e 2 componenti. L'ingresso $u^{0}$ si propaga nella rete mediante le seguenti regole:

$$
\begin{array}{lccc} 
& \eta^{1}=\bar{u}^{0} \bar{W}^{1} & \eta^{2}=\bar{u}^{1} \bar{W}^{2} & \eta^{3}=\bar{u}^{2} \bar{W}^{3} \\
u^{1}=s\left(\eta^{1}\right) & u^{2}=s\left(\eta^{2}\right) & u^{3}=\eta^{3} \\
\bar{u}^{0}=\left(u^{0}, 1\right) & \bar{u}^{1}=\left(u^{1}, 1\right) & \bar{u}^{2}=\left(u^{2}, 1\right) &
\end{array}
$$

che definiscono l'uscita $u^{3}$ delle rete, per cui $u^{3}=F\left(u_{0}\right)$.

Possiamo adattare la rete descritta ad un insieme di dati, in modo da poterla usare come strumento predittivo. L'estrema semplicità dei componenti elementari ed il grande numero di parametri disponibili rendono una rete neurale molto conveniente per questo scopo. Inoltre una rete neurale non richiede una scelta preventiva del tipo di funzioni da usare, anche se è possibile specializzare i neuroni in modo da fornire determinate caratteristiche. Ad esempio si possono usare i neuroni a coppie, definendo i così detti neuroni circolari, nel caso in cui i dati da analizzare presentino delle periodicità [14]. Una coppia di neuroni circolari produce un'uscita che, per ciascun neurone, dipende oltre che dai propri ingressi anche dagli ingressi associati.

Più precisamente, come rappresentato nella Figura 7, se $\xi$ è l'ingresso del primo neurone e $\eta$ è l'ingresso del secondo, allora il primo ed il secondo neurone produrranno un'uscita, rispettivamente pari a: $\frac{\xi}{\sqrt{\xi^{2}+\eta^{2}}}, \frac{\eta}{\sqrt{\xi^{2}+\eta^{2}}}$.

Si ottiene quindi che la funzione di attivazione $s$ dipende da due variabili ed assume valori in $\mathbb{R}^{2}: s(x, y)=\left(\frac{x}{\sqrt{x^{2}+y^{2}}}, \frac{y}{\sqrt{x^{2}+y^{2}}}\right)$. L'algoritmo di backpropagation dipenderà da: $\nabla s(x, y)=\left(\begin{array}{cc}\frac{x^{2}}{\sqrt{\left(x^{2}+y^{2}\right)^{3}}} & -\frac{x y}{\sqrt{\left(x^{2}+y^{2}\right)^{3}}} \\ -\frac{x y}{\sqrt{\left(x^{2}+y^{2}\right)^{3}}} & \frac{x^{2}}{\sqrt{\left(x^{2}+y^{2}\right)^{3}}}\end{array}\right)$.

Addestrare la rete significa determinare i parametri che la definiscono, cioè le matrici dei pesi $W^{k}$ e i vettori bias $b^{k}$, in modo che la differenza tra uscita della rete e valore atteso sia minima. In altre parole, se si vuole adattare la rete affinchè rappresenti una funzione che ricevendo in ingresso i valori $\left\{x_{j}\right\}$ restituisca i valori $\left\{t_{j}\right\}$, si devono scegliere $W^{k}$ e $b^{k}$, in modo che detti $o_{j}$ i risultati in uscita dalla rete che corrispondono agli ingressi $x_{j}$, sia minimo lo scarto quadratico tra $o_{j}$ e $t_{j}$.

$$
E=\frac{1}{2} \sum_{j=1}^{N}\left(o_{j}-t_{j}\right)^{2}
$$

Dove $N$ è il numero degli elementi nel training set della rete neurale.

Evidentemente $E$ risulta funzione dei coefficienti della rete e, per gli scopi enunciati, è chiaro che occorre trovarne il minimo. Ciò può essere ottenuto mediante il metodo del gradiente e, nonostante la apparente complessità dei calcoli necessari per ottenere le derivate della funzione $E$ rispetto ad ognuno dei parametri da cui dipende, si verifica che esistono semplici formule localizzate per descriverle. L'algoritmo mediante il quale si aggiornano i coefficienti della rete si indica di solito col nome di back-propagation in virtù del fatto che lo scarto registrato in corrispondenza di un certo dato viene fatto propagare all'indietro nella rete per ottenere le formule di aggiornamento dei coefficienti [6]. 

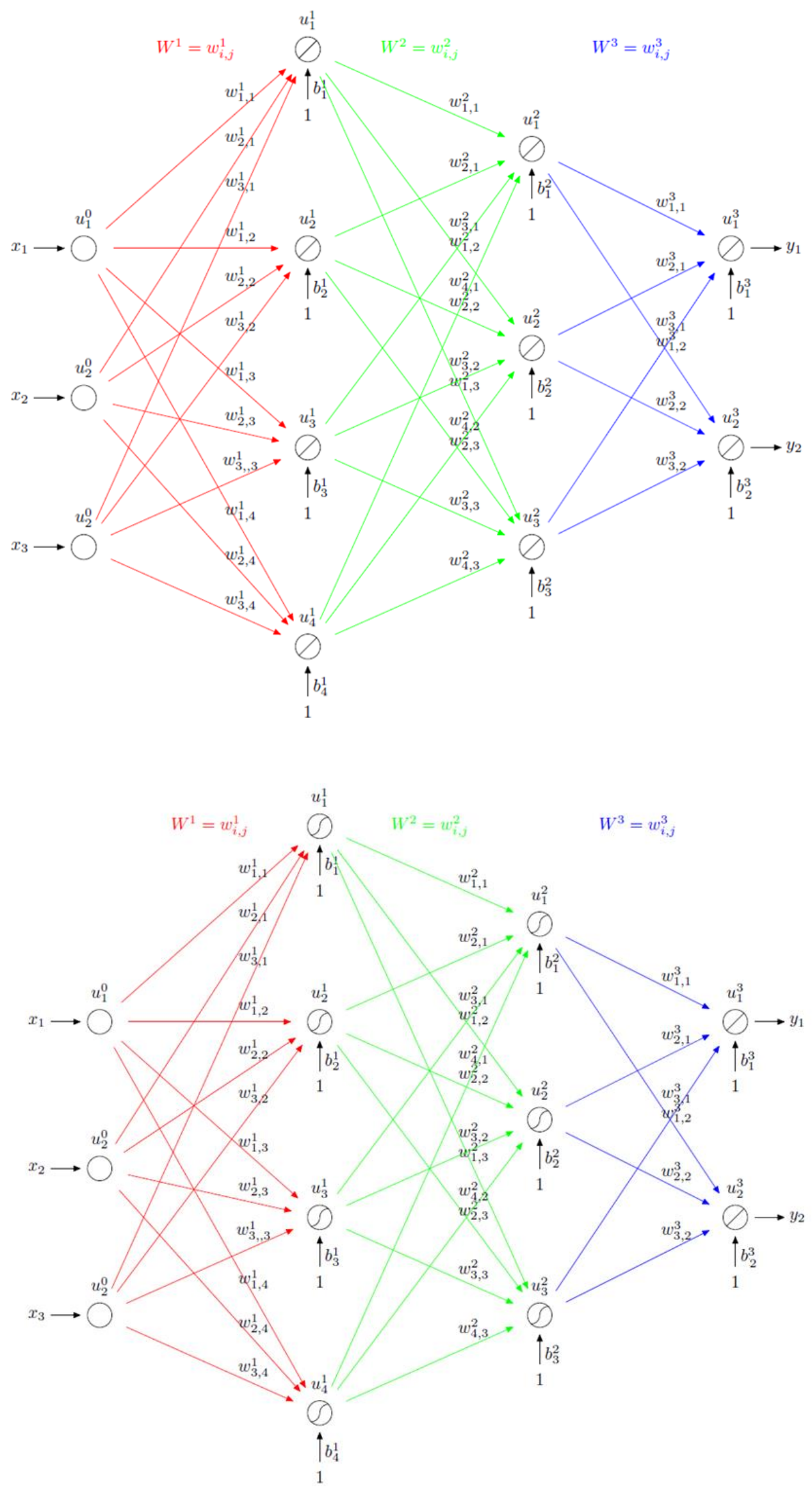

Figura 6. Rete neurale lineare e non lineare: nella prima la funzione di attivazione dei neuroni degli strati interni è lineare, mentre nella seconda è non lineare 


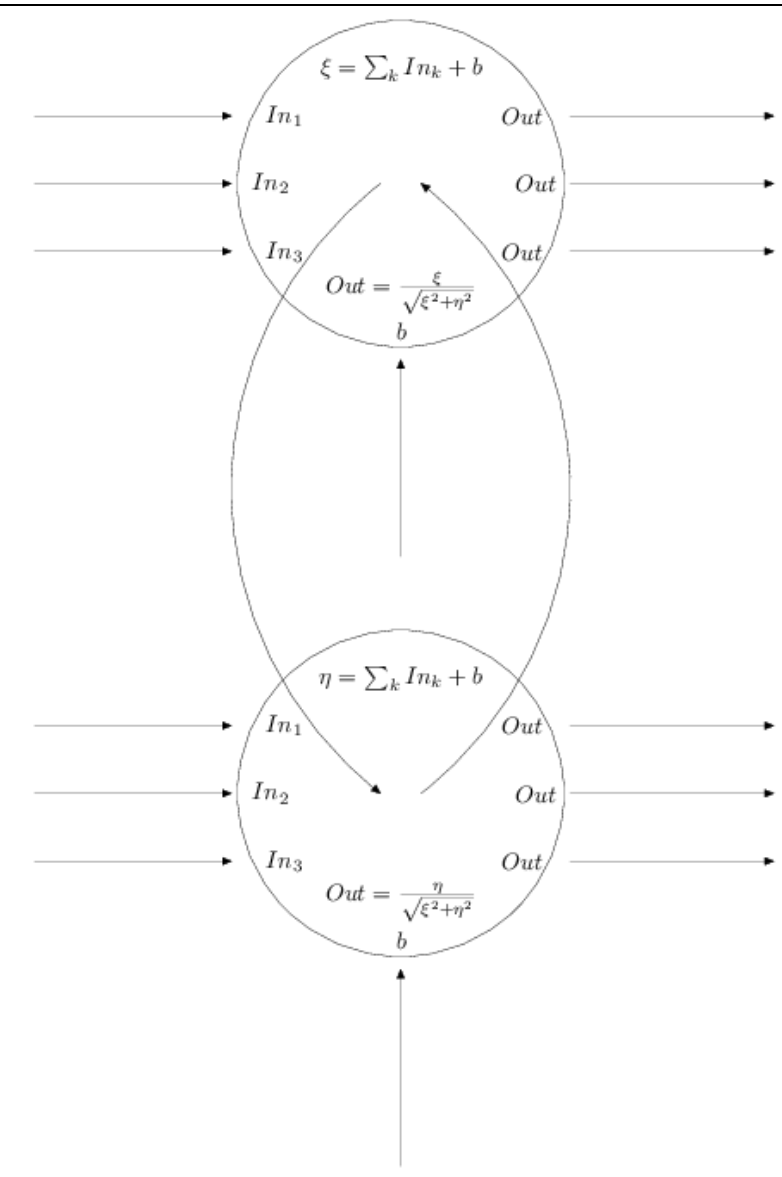

Figura 7. Rappresentazione dei Neuroni circolari

Per illustrare l'algoritmo di back-propagation consideriamo il caso della rete in Figura 6. In corrispondenza di ciascun $\left(x_{j}, t_{j}\right)$ per $j=1, \ldots, N$ possiamo quindi porre $u^{0}=x_{j}$, calcolare la corrispondente uscita $\eta_{3}=o_{j}$ e stimare l'errore mediante la (29). Per applicare il metodo del gradiente occorre calcolare le derivate parziali di $E: \nabla E=\left(\frac{\partial E}{\partial \bar{W}^{1}}, \frac{\partial E}{\partial \bar{W}^{2}}, \frac{\partial E}{\partial \bar{W}^{3}}\right)$.

Usando la regola di derivazione delle funzioni composte, si ottiene:

$$
\begin{gathered}
\frac{\partial E}{\partial \bar{W}^{3}}=\frac{\partial E}{\partial u^{3}} \frac{\partial u^{3}}{\partial \bar{W}^{3}} \\
\frac{\partial E}{\partial \bar{W}^{2}}=\frac{\partial E}{\partial u^{3}} \frac{\partial u^{3}}{\partial u^{2}} \frac{\partial u^{2}}{\partial \bar{W}^{2}} \\
\frac{\partial E}{\partial \bar{W}^{1}}=\frac{\partial E}{\partial u^{3}} \frac{\partial u^{3}}{\partial u^{2}} \frac{\partial u^{2}}{\partial u^{1}} \frac{\partial u^{1}}{\partial \bar{W}^{1}}
\end{gathered}
$$

Ed è facile verificare che:

$$
\begin{gathered}
\frac{\partial E}{\partial \bar{W}^{3}}=\left(o_{j}-t_{j}\right) \frac{\partial u^{3}}{\partial \bar{W}^{1}}=\left(u_{3}-t_{j}\right) \frac{\partial \eta^{3}}{\partial \bar{W}^{1}} \\
\frac{\partial \eta^{3}}{\partial \bar{W}^{3}}=\left(\bar{u}^{2}\right)^{T}
\end{gathered}
$$

in quanto $\eta^{3}=\bar{u}^{2} \bar{W}^{3}$ è funzione lineare di $\bar{W}^{3}$. Per calcolare le altre derivate, posto:

$$
D_{2}=\left(\begin{array}{ccc}
u_{1}^{2}\left(1-u_{1}^{2}\right) & 0 & 0 \\
0 & u_{2}^{2}\left(1-u_{2}^{2}\right) & 0 \\
0 & 0 & u_{3}^{2}\left(1-u_{3}^{2}\right)
\end{array}\right)
$$




$$
D_{1}=\left(\begin{array}{cccc}
u_{1}^{1}\left(1-u_{1}^{1}\right) & 0 & 0 & 0 \\
0 & u_{2}^{1}\left(1-u_{2}^{1}\right) & 0 & 0 \\
0 & 0 & u_{3}^{1}\left(1-u_{3}^{1}\right) & 0 \\
0 & 0 & 0 & u_{4}^{1}\left(1-u_{4}^{1}\right)
\end{array}\right)
$$

Si ha:

$$
\begin{gathered}
\frac{\partial E}{\partial \bar{W}^{2}}=\frac{\partial E}{\partial u^{3}} \frac{\partial u^{3}}{\partial u^{2}} \frac{\partial u^{2}}{\partial \bar{W}^{2}}=D_{2} W^{3} \bar{u}^{2} \\
\frac{\partial E}{\partial \bar{W}^{1}}=\frac{\partial E}{\partial u^{3}} \frac{\partial u^{3}}{\partial u^{2}} \frac{\partial u^{2}}{\partial u^{1}} \frac{\partial u^{1}}{\partial \bar{W}^{1}}=D_{1} W^{2} D_{2} W^{3} \bar{u}^{0}
\end{gathered}
$$

Infine se $\delta^{3}=\left(u_{3}-t\right), \delta^{2}=D_{2} W^{3}, \delta^{1}=D_{1} W^{2}$ possiamo scrivere gli aggiornamenti dettati dal metodo del gradiente nella seguente maniera:

$$
\begin{gathered}
\bar{W}^{3}=\bar{W}^{3}-l_{r}\left(\delta^{3} \bar{u}^{2}\right)^{T} \\
\bar{W}^{2}=\bar{W}^{2}-l_{r}\left(\delta^{2} \delta^{3} \bar{u}^{1}\right)^{T} \\
\bar{W}^{3}=\bar{W}^{3}-l_{r}\left(\delta^{1} \delta^{2} \delta^{3} \bar{u}^{0}\right)^{T}
\end{gathered}
$$

dove $l_{r}$ è il passo con cui il metodo del gradiente avanza ed è, in questo ambito, chiamato coefficiente di apprendimento: learning rate.

Minimizzare l'errore globale ottenuto sommando gli scarti quadratici in corrispondenza di ogni punto del training set, si identifica col procedere ad un aggiornamento off-line dei coefficienti. Nel caso si consideri ripetutamente l'errore ottenuto da un solo scarto quadratico, invece l'aggiornamento viene fatto on-line. Nell'ultimo caso, spesso preferito nelle applicazioni, i punti del training set vengono proposti più volte alla rete neurale nel corso del suo apprendimento.

Al fine di validare il codice scritto in Matlab, che implementa tale architettura di rete neurale feed-forward con neuroni circolari, si pone come obiettivo l'individuazione della periodicità su una serie storica di dati rappresentativi del carico elettrico del Campus Universitario di Savona - Sede decentrata dell’Università di Genova, come descritto in [1].

Considerando una rete neurale a tre strati per approssimare la time-series graficata in verde ed in rosso nella Figura 8, si riescono ad estrapolare correttamente i valori futuri (in blu), assicurando una predizione robusta ed affidabile, in quanto la struttura periodica risulta chiaramente individuata. Il training set della rete in questo caso è costituito esclusivamente dai valori in verde.

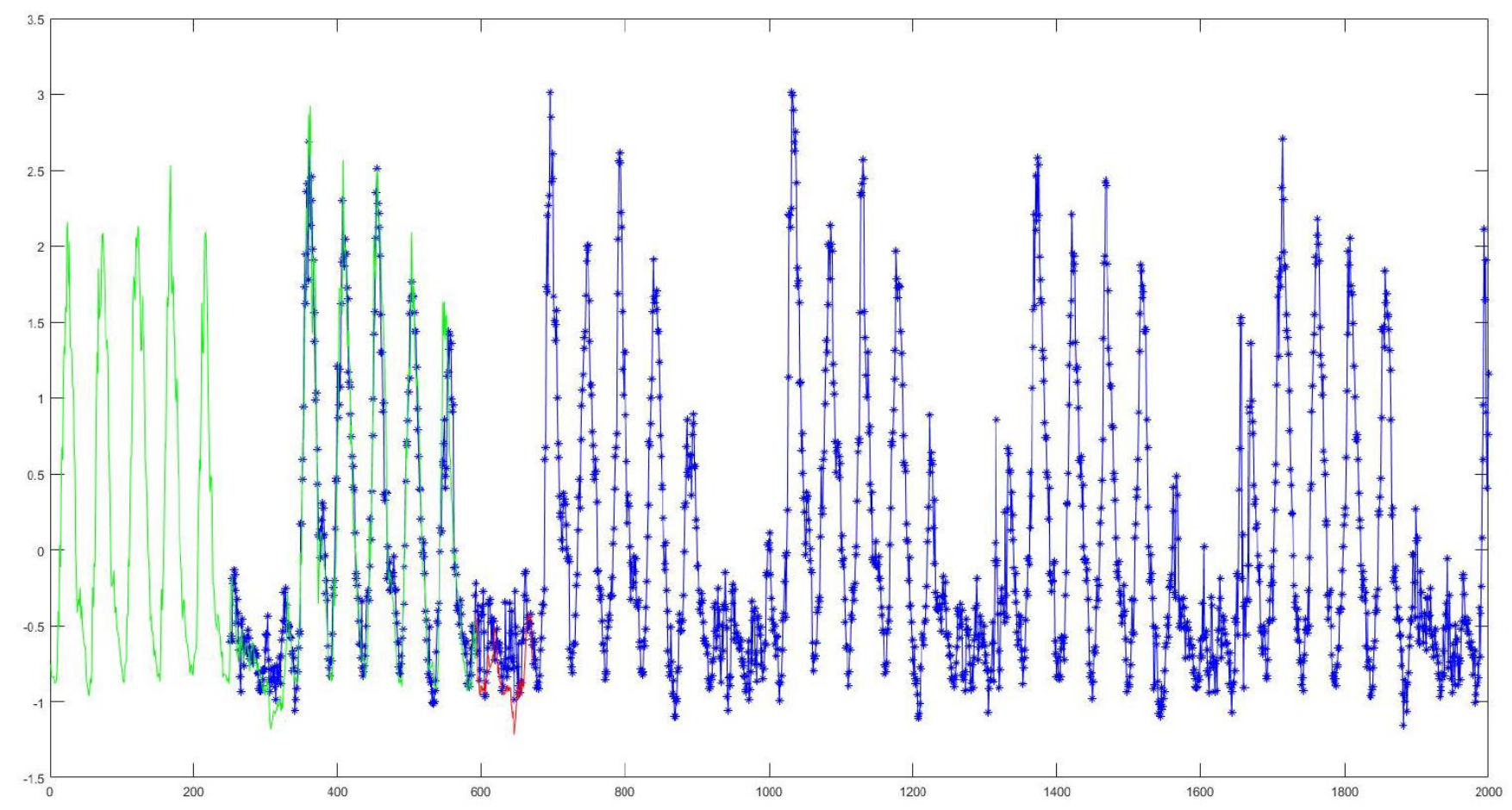

Figura 8. Forecasting sul data set di validazione per la ANN feed-forward con neuroni circolari 


\section{Confronto tra le due metodologie di simulazione dei valori prospettici dell'indice CPI}

Al fine di comparare gli approcci metodologici di bootstrap dei valori inflattivi, si considerino i dati di mercato di venerdì 29 giugno 2018 (Fonte Bloomberg®).

I valori degli swap rate $K\left(T_{M}\right)$ quotati dal mercato alla data di riferimento sono riportati nella Tabella 1 e sintetizzati sino a $T_{M}=5$ anni nella Tabella 2 , unitamente alla stima degli $\mathfrak{J}_{M}(0)$ come descritto dalla formula (11)

\begin{tabular}{|c|c|c|c|c|c|}
\hline Year $T_{M}$ & Bid Value & Ask Value & Mid $K\left(T_{M}\right)$ & $\mathfrak{I}_{M}(0)$ & $\Delta \widetilde{I}_{M}$ \\
\hline 1 & 1,67 & 1,69 & 1,680 & 104.8422 & 0.001369 \\
\hline 2 & 1,53 & 1,58 & 1,555 & 106.3417 & 0.001164 \\
\hline 3 & 1,49 & 1,57 & 1,526 & 107.9028 & 0.001198 \\
\hline 4 & 1,50 & 1,55 & 1,525 & 109.5451 & 0.001242 \\
\hline 5 & 1,51 & 1,54 & 1,528 & 111.2321 & 0.001256 \\
\hline
\end{tabular}

Tabella 2. Swap Rate quotati sull'inflazione (bid-ask) del 29 giugno $2018, K\left(T_{M}\right), \mathfrak{I}_{M}(0)$ e $\Delta \mathfrak{I}_{M}$

Al fine di stimare i valori inflattivi con una periodicità pari a quella di rilevazione, ovvero mensile anziché annuale, si ricava dalla (25) gli incrementi inflattivi mensili $\Delta \mathfrak{I}_{M}$ da applicare all'interno degli anni considerati, così come riportati nella Tabella 2. Queste informazioni sono impiegabili per stimare, in accordo alla (26), i valori prospettici della CPI per i prossimi 5 anni senza tenere conto della stagionalità. Al fine di aggiungere tale essenziale contributo al modello dei valori prospettici della CPI, è necessario disporre della serie storica per il calcolo dei residui normalizzati mensili $\mathfrak{R}_{M}$.

La Tabella 3 mostra i valori di $\mathfrak{R}_{M}$ stimati in accordo con la (27)

\begin{tabular}{|c|c|c|c|c|c|c|c|c|c|c|c|c|}
\hline Month & 1 & 2 & 3 & 4 & 5 & 6 & 7 & 8 & 9 & 10 & 11 & 12 \\
\hline $\mathfrak{R}_{M}$ & $-1,2571$ & 0,2882 & 0,9738 & 0,0981 & 0,0429 & 0,0266 & $-0,6507$ & 0,0694 & 0,3509 & 0,0146 & $-0,1444$ & 0,1879 \\
\hline
\end{tabular}

Tabella 3. I residui normalizzati mensili, $\mathfrak{R}_{M}$

Applicando ricorsivamente l'equazione (26), si ricavano le proiezioni della CPI per i successivi 5 anni [18]: i risultati sono riportati nella Figura 9, congiuntamente ai valori passati dell'indice. In particolare la linea rossa, dopo l'ultimo valore registrato dall'inflazione (in corrispondenza di aprile 2018), rappresenta la simulazione di $\mathfrak{J}_{i}$ senza il contributo della stagionalità (ovvero ponendo $\mathfrak{R}_{M}=0$ ); per contro la linea blu rappresenta la proiezione di $\mathfrak{I}_{i}$ impiegando i residui normalizzati della Tabella 3 .

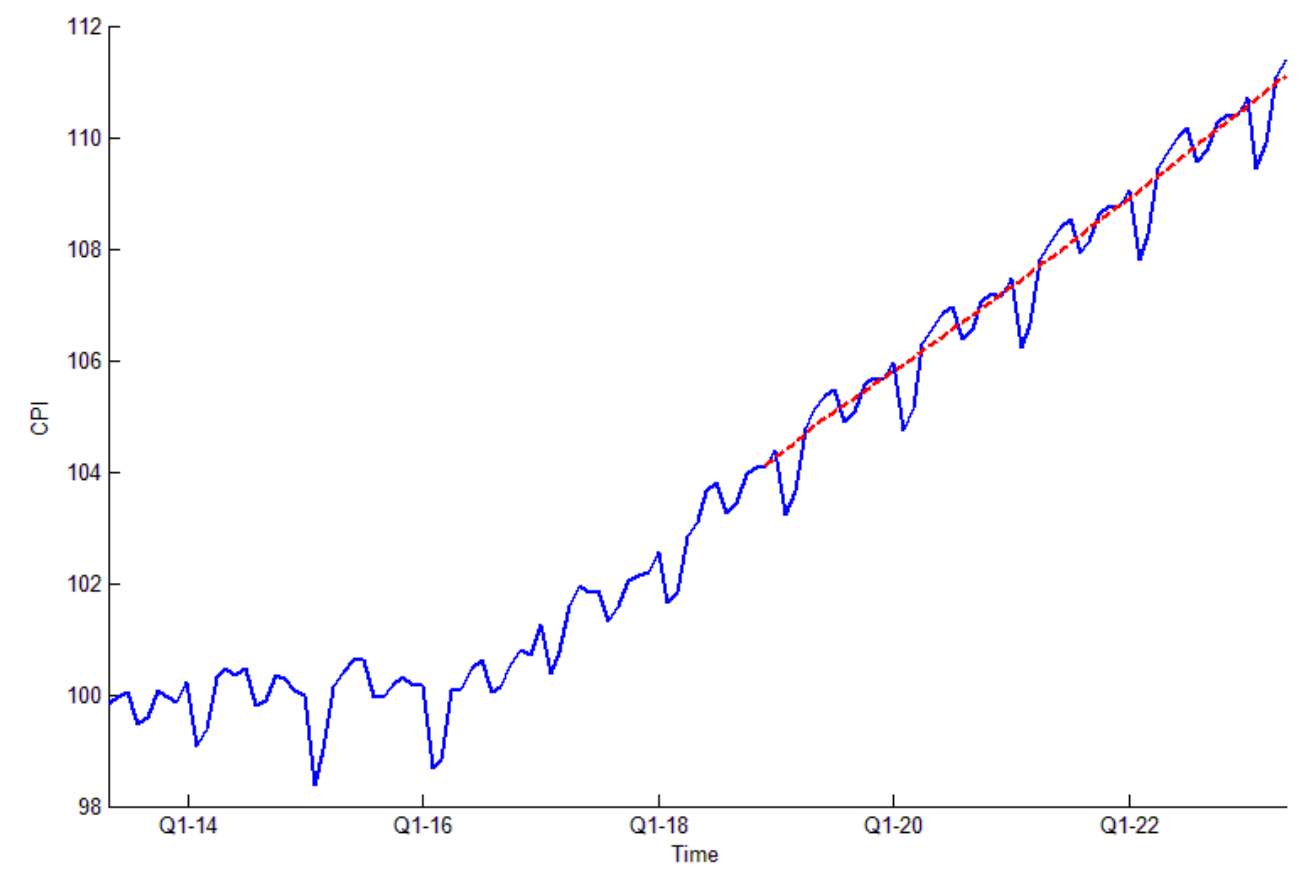

Figura 9. Serie storica dell'indice inflattivo europeo (maggio2013 - maggio 2018) e relativa proiezione 
Con l'approccio di Machine Learning viene impiegata per la simulazione prospettica dei valori inflattivi la formula ricorsiva (26) nella quale, al posto di impiegare gli $\mathfrak{R}_{M}$ utilizzati per tenere conto della stagionalità mensile, vengono impiegati i valori estrapolati dalla rete neurale feed-forward con neuroni circolari, a partire dal dataset dei logaritmi dei rendimenti ln $\left[\frac{\Im_{i+1}^{\text {Monthly }}}{\mathfrak{\Im}_{i}^{\text {Monthly }}}\right]$ registrati negli ultimi 5 anni dall'indice inflattivo europeo. Il vantaggio di impiegare questa tecnologia consente, rispetto al metodo di calcolo precedente, di effettuare previsioni ragionevolmente più robuste e di evitare l'artificiosa procedura di aggiungere per ogni mese dell'anno simulato il medesimo incremento $\mathfrak{R}_{M}$.

La Figura 10 riporta i grafici dei logaritmi dei rendimenti storici dal maggio 2013 (indicizzato con -60 , ovvero 60 mesi indietro rispetto all'attuale rilevazione) al maggio 2018 (index 0) e quelli prospettici (a destra della linea verticale rossa) sino al maggio 2023 (indicizzato con +60 , ovvero 60 mesi in avanti rispetto all'attuale rilevazione), stimati con la metodologia standard.

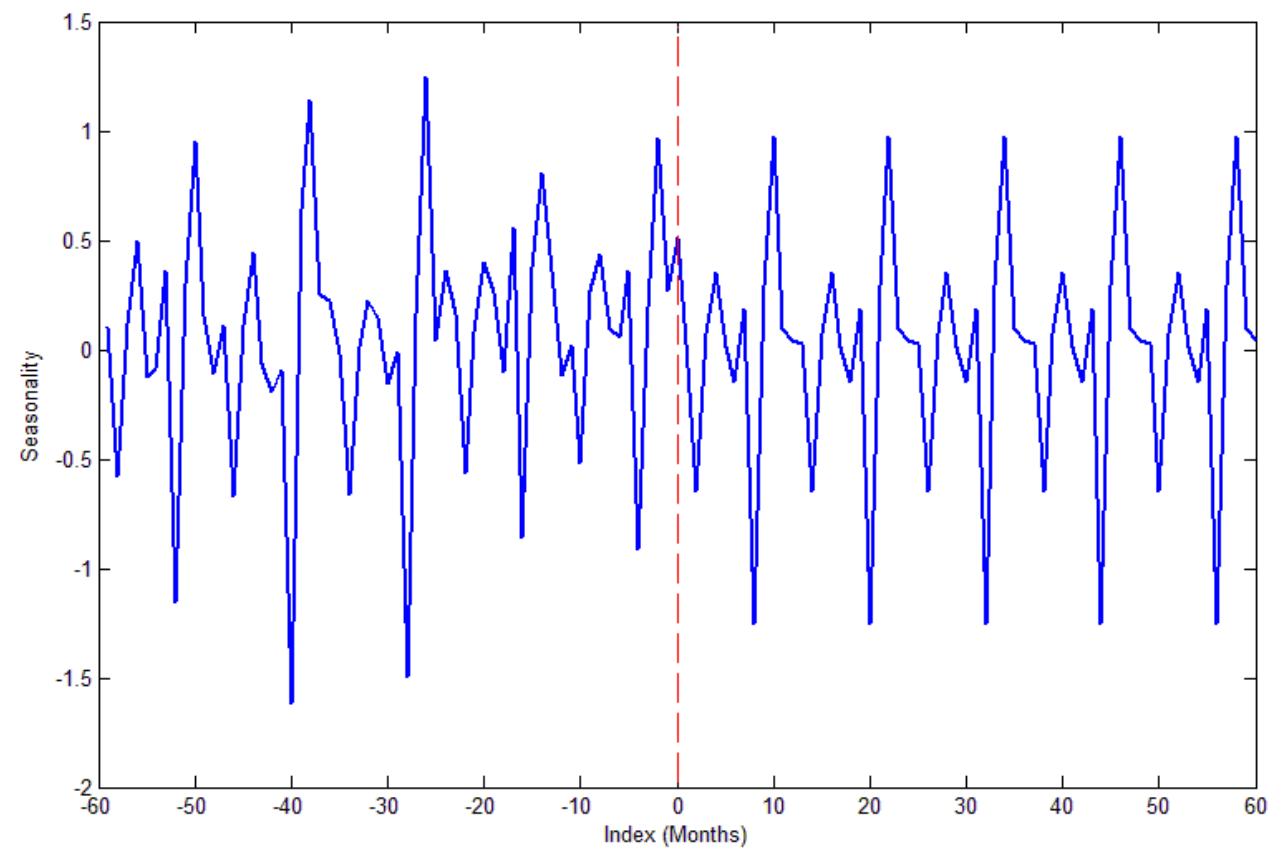

Figura 10. Stagionalità storica e prospettica stimata con la metodologia standard

La Figura 11 riporta i grafici dei logaritmi dei rendimenti storici dal maggio 2013 al maggio 2018 e quelli prospettici sino al maggio 2023, stimati con la rete neurale feed-forward con neuroni circolari.

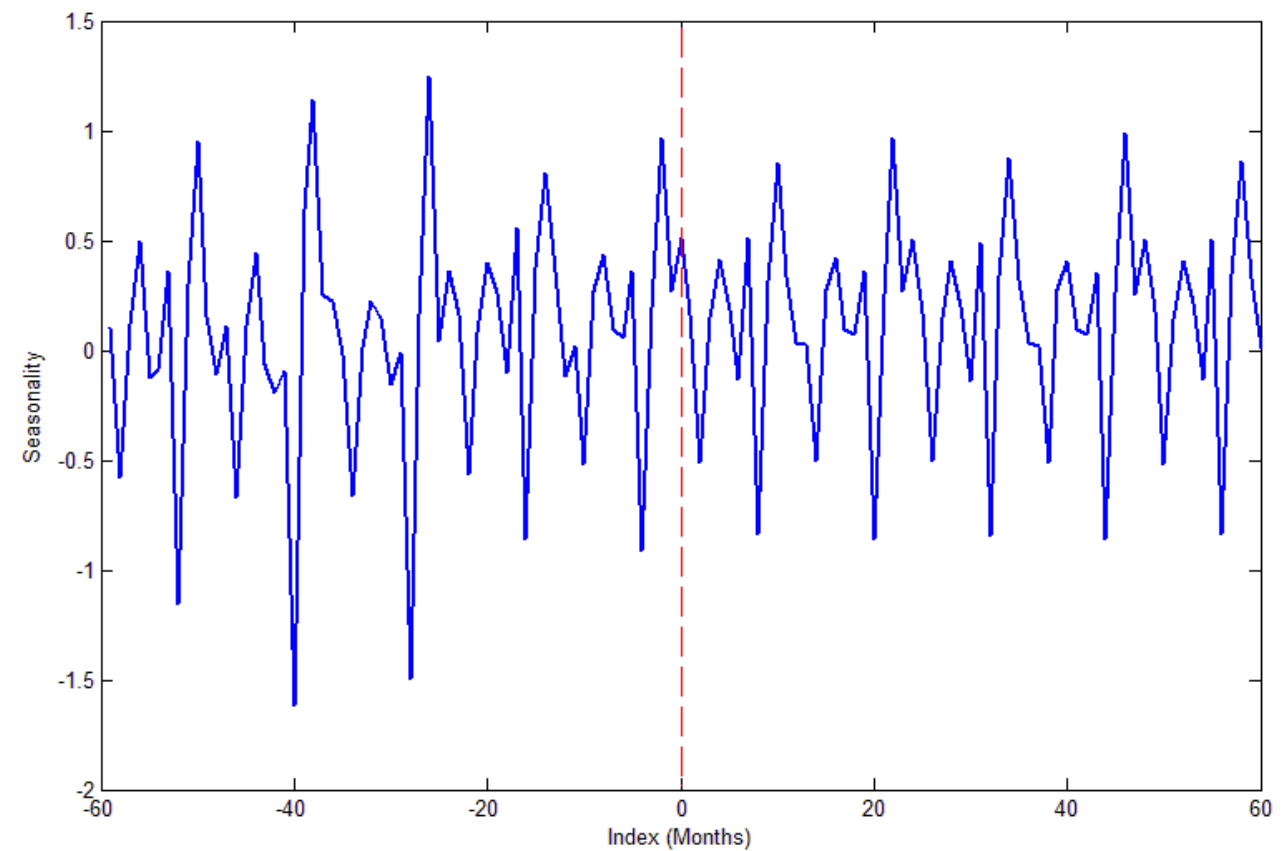

Figura 11. Stagionalità storica e prospettica stimata con la metodologia di Machine Learning

Considerando la nuova stagionalità $\mathfrak{R}$, si possono ottenere i valori della CPI prospettici sempre a partire dalla relazione (26). 


\section{Pricing di uno swap di tipo YYIIS indicizzato all’inflazione}

Presentati nel paragrafo precedente le due modalità per ricavare i valori prospettici della CPI, si procede ora alla valorizzazione di uno swap di tipo YYIIS, avente le caratteristiche riportate nella Tabella 4.

\begin{tabular}{|c|c|c|}
\hline & Receive Leg & Pay Leg \\
\hline Leg Type & Y-o-Y Inflation & Fixed \\
\hline Notional & 10.000 .000 & 10.000 .000 \\
\hline Currency & EUR & EUR \\
\hline Index & CPTFEMU Index & Fixed Coupon 1\% \\
\hline Effective Date & $30 / 08 / 2018$ & $30 / 08 / 2018$ \\
\hline Maturity Date & $30 / 08 / 2023$ & $30 / 08 / 2023$ \\
\hline Lag & 3 Months & \\
\hline Interpolation & Monthly & \\
\hline Spread & 0 & \\
\hline Reset Freq & Semi-Annual & Semi-Annual \\
\hline Payment Freq & Semi-Annual & ACT/365 \\
\hline Day Count & ACT/ACT & EUR - OIS \\
\hline Disc Curve & EUR - OIS & \\
\hline Fwd Curve & European Inflation Curve & \\
\hline
\end{tabular}

Tabella 4. Caratteristiche finanziarie dello YYIIS

La data di valutazione dello swap "In Arrears" è il 29 giugno 2018, per cui i dati inerenti l'indice inflattivo sono già quelli riportati nei prospetti e nei grafici precedenti. La struttura a termine dei tassi d'interesse impiegata per lo sconto dei flussi di cassa è l'EUR OIS, i cui Par Rates, Zero Rates e Discount Factors sono sintetizzati nella Tabella 5.

\begin{tabular}{|c|c|c|c|}
\hline Term & Par Rate & Zero Rate & Discount \\
\hline $1 \mathrm{DY}$ & $-0,358$ & $-0,358$ & 1,00001 \\
\hline $2 \mathrm{WK}$ & $-0,36$ & $-0,35938$ & 1,00014 \\
\hline $1 \mathrm{MO}$ & $-0,359$ & $-0,35841$ & 1,000309 \\
\hline $2 \mathrm{MO}$ & $-0,35775$ & $-0,35722$ & 1,000617 \\
\hline $3 \mathrm{MO}$ & $-0,3569$ & $-0,35643$ & 1,000913 \\
\hline $4 \mathrm{MO}$ & $-0,35775$ & $-0,35733$ & 1,001244 \\
\hline $5 \mathrm{MO}$ & $-0,357$ & $-0,35663$ & 1,00152 \\
\hline $6 \mathrm{MO}$ & $-0,357$ & $-0,35669$ & 1,001828 \\
\hline $7 \mathrm{MO}$ & $-0,357$ & $-0,35674$ & 1,002147 \\
\hline $8 \mathrm{MO}$ & $-0,356$ & $-0,3558$ & 1,002419 \\
\hline $9 \mathrm{MO}$ & $-0,356$ & $-0,35585$ & 1,002717 \\
\hline $10 \mathrm{MO}$ & $-0,355$ & $-0,3549$ & 1,003007 \\
\hline $11 \mathrm{MO}$ & $-0,355$ & $-0,35496$ & 1,003314 \\
\hline $12 \mathrm{MO}$ & $-0,35375$ & $-0,35376$ & 1,0036 \\
\hline $18 \mathrm{MO}$ & $-0,338$ & $-0,33795$ & 1,005176 \\
\hline $2 \mathrm{YR}$ & $-0,3$ & $-0,30009$ & 1,006121 \\
\hline $30 \mathrm{MO}$ & $-0,244$ & $-0,24419$ & 1,00624 \\
\hline $3 \mathrm{YR}$ & $-0,179$ & $-0,17926$ & 1,005487 \\
\hline $4 \mathrm{YR}$ & $-0,0465$ & $-0,04664$ & 1,001897 \\
\hline $5 \mathrm{YR}$ & 0,087 & 0,08745 & 0,995576 \\
\hline $6 \mathrm{YR}$ & 0,217 & 0,21867 & 0,986788 \\
\hline
\end{tabular}

Tabella 5. EUR-OIS Term Structure - 29 Giugno 2018 - Fonte: Bloomberg® 
Impiegando l'approccio metodologico di pricing presentato nel paragrafo 2, si stimano i flussi di cassa, ottenendo il NPV della gamba pagare e ricevere dello swap in esame [16]. In particolare si osserva:

- nella Tabella 6 sono riportati i cash flow scontati per la gamba pagare fissa dello swap.

- nella Tabella 7 sono riportati i cash flow scontati per la gamba ricevere indicizzata all'inflazione con la stima dei valori prospettici della CPI, impiegando il calcolo della stagionalità effettuato con la metodologia standard (Fig. 11).

- nella Tabella 8 sono riportati i cash flow scontati per la gamba ricevere indicizzata all'inflazione, con la stima dei valori prospettici della CPI, impiegando il calcolo della stagionalità effettuato con la metodologia di machine learning (Fig. 12).

\begin{tabular}{|c|c|c|c|c|c|c|}
\hline Accrual Start & Accrual End & Days & Notional & Payment & Discount & Present Value \\
\hline $08 / 30 / 2018$ & $02 / 28 / 2019$ & 182 & -10000000 & $-49863,01$ & 1,00241 & $-49983,18$ \\
\hline $02 / 28 / 2019$ & $08 / 30 / 2019$ & 183 & -10000000 & $-50136,99$ & 1,004141 & $-50344,60$ \\
\hline $08 / 30 / 2019$ & $02 / 28 / 2020$ & 182 & -10000000 & $-49863,01$ & 1,00553 & $-50138,76$ \\
\hline $02 / 28 / 2020$ & $08 / 31 / 2020$ & 185 & -10000000 & $-50684,93$ & 1,006258 & $-51002,12$ \\
\hline $08 / 31 / 2020$ & $02 / 26 / 2021$ & 179 & -10000000 & $-49041,10$ & 1,006133 & $-49341,86$ \\
\hline $02 / 26 / 2021$ & $08 / 30 / 2021$ & 185 & -10000000 & $-50684,93$ & 1,005156 & $-50946,26$ \\
\hline $08 / 30 / 2021$ & $02 / 28 / 2022$ & 182 & -10000000 & $-49863,01$ & 1,003492 & $-50037,14$ \\
\hline $02 / 28 / 2022$ & $08 / 30 / 2022$ & 183 & -10000000 & $-50136,99$ & 1,001136 & $-50193,94$ \\
\hline $08 / 30 / 2022$ & $02 / 28 / 2023$ & 182 & -10000000 & $-49863,01$ & 0,9981 & $-49768,27$ \\
\hline $02 / 28 / 2023$ & $08 / 30 / 2023$ & 183 & -10000000 & $-50136,99$ & 0,994411 & $-49856,77$ \\
\hline & & & & & NPV Leg & $-501612,91$ \\
\hline
\end{tabular}

Tabella 6. Flussi di cassa della gamba pagare dello swap YoY

\begin{tabular}{|c|c|c|c|c|c|c|c|}
\hline Accrual Start & Accrual End & Days & Notional & Reset Date & Rate [\%] & Payment & PV \\
\hline $08 / 30 / 2018$ & $02 / 28 / 2019$ & 182 & 10000000 & Nov 2018 & 0,4114122 & 41141,22 & 41240,37 \\
\hline $02 / 28 / 2019$ & $08 / 30 / 2019$ & 183 & 10000000 & May 2019 & 1,2194895 & 121948,95 & 122453,94 \\
\hline $08 / 30 / 2019$ & $02 / 28 / 2020$ & 182 & 10000000 & Nov 2019 & 0,2877428 & 28774,28 & 28933,40 \\
\hline $02 / 28 / 2020$ & $08 / 31 / 2020$ & 185 & 10000000 & May 2020 & 1,1190166 & 111901,66 & 112601,94 \\
\hline $08 / 31 / 2020$ & $02 / 26 / 2021$ & 179 & 10000000 & Nov 2020 & 0,3081261 & 30812,61 & 31001,58 \\
\hline $02 / 26 / 2021$ & $08 / 30 / 2021$ & 185 & 10000000 & May 2021 & 1,1405639 & 114056,39 & 114644,47 \\
\hline $08 / 30 / 2021$ & $02 / 28 / 2022$ & 182 & 10000000 & Nov 2021 & 0,3344362 & 33443,62 & 33560,40 \\
\hline $02 / 28 / 2022$ & $08 / 30 / 2022$ & 183 & 10000000 & May 2022 & 1,1641446 & 116414,46 & 116546,71 \\
\hline $08 / 30 / 2022$ & $02 / 28 / 2023$ & 182 & 10000000 & Nov 2022 & 0,3432091 & 34320,91 & 34255,70 \\
\hline $02 / 28 / 2023$ & $08 / 30 / 2023$ & 183 & 10000000 & May 2023 & 1,1785192 & 117851,92 & 117193,25 \\
\hline
\end{tabular}

Tabella 7. Flussi di cassa della gamba ricevere dello swap YoY (metodologia per la stagionalità standard)

\begin{tabular}{||c|c|c|c|c|c|c|c|}
\hline Accrual Start & Accrual End & Days & Notional & Infl. Reset Date & Rate [\%] & Payment & PV \\
\hline $08 / 30 / 2018$ & $02 / 28 / 2019$ & 182 & 10000000 & $11 / 01 / 2018$ & 0,3114122 & 31141,22 & 31216,27 \\
\hline $02 / 28 / 2019$ & $08 / 30 / 2019$ & 183 & 10000000 & $05 / 01 / 2019$ & 1,3940048 & 139400,48 & 139977,73 \\
\hline $08 / 30 / 2019$ & $02 / 28 / 2020$ & 182 & 10000000 & $11 / 01 / 2019$ & 0,3866633 & 38666,33 & 38880,16 \\
\hline $02 / 28 / 2020$ & $08 / 31 / 2020$ & 185 & 10000000 & $05 / 01 / 2020$ & 1,3055187 & 130551,87 & 131368,87 \\
\hline $08 / 31 / 2020$ & $02 / 26 / 2021$ & 179 & 10000000 & $11 / 01 / 2020$ & 0,2081261 & 20812,61 & 20940,25 \\
\hline $02 / 26 / 2021$ & $08 / 30 / 2021$ & 185 & 10000000 & $05 / 01 / 2021$ & 1,3148273 & 131482,73 & 132160,66 \\
\hline $08 / 30 / 2021$ & $02 / 28 / 2022$ & 182 & 10000000 & $11 / 01 / 2021$ & 0,7076675 & 70766,75 & 71013,87 \\
\hline $02 / 28 / 2022$ & $08 / 30 / 2022$ & 183 & 10000000 & $05 / 01 / 2022$ & 1,3680161 & 136801,61 & 136957,02 \\
\hline $08 / 30 / 2022$ & $02 / 28 / 2023$ & 182 & 10000000 & $11 / 01 / 2022$ & 0,3432091 & 34320,91 & 34255,70 \\
\hline $02 / 28 / 2023$ & $08 / 30 / 2023$ & 183 & 10000000 & $05 / 01 / 2023$ & 1,376496 & 137649,60 & 136880,28 \\
\hline & & & & & NPV Leg & 873650,81 \\
\hline
\end{tabular}

Tabella 8. Flussi di cassa della gamba ricevere dello swap YoY (metodologia per la stagionalità Machine Learning) 
Modellizzando la stagionalità dell'inflazione in accordo con la metodologia base, si ottiene un valore per lo swap Y-O-Y pari a $N P V_{L E G R E C}-N P V_{L E G ~ P A Y}=752.431,76-501.612,91=+250.818,85$, mentre eseguendo il pricing del derivato impiegando la rete neurale per la stagionalità si ottiene un valore pari a: $N P V_{L E G ~ R E C}-N P V_{L E G ~ P A Y}=873.650,81-$ $501.612,91=+372.037,90$. L'impiego di un differente modello per interpretare la stagionalità dell'indice CPI pone in evidenza una discrepanza significativa di pricing $(121.219,05)$, pari ad un gap percentuale rapportato al nozionale superiore all' $1 \%$.

\section{Conclusioni}

Il paper ha mostrato in che modo una metodologia di Machine Learning può essere implementata in un framework di pricing finalizzato alla determinazione del fair-value di derivati legati all'indice inflattivo. La rete neurale feed-forward, dotata di neuroni circolari, ha consentito di identificare in modo più affidabile l'effetto della stagionalità rispetto al metodo tradizionale base. Si ritiene particolarmente interessante come l'individuazione di tale periodicità, ad opera dell'intelligenza artificiale, possa essere utilmente integrata con gli approcci metodologici tradizionali di pricing propri della finanza quantitativa.

Si ritiene interessante, per il proseguo dello studio applicare la tecnologia proposta a derivati scritti su altri asset, in cui la modellizzazione della stagionalità è di fondamentale importanza: si pensi, a titolo di esempio, ai derivati sulle commodity e sull'energia.

\section{Ottavio Caligaris e Pier Giuseppe Giribone}

\section{Bibliografia}

[1] Bracco S., Delfino F., Pampararo F., Robba M., Rossi M., "A pilot facility for analysis and simulation of smart microgrids feeding smart buildings", Renewable and Sustainable Energy Reviews, Vol. 58, 1247-1255 (2016)

[2] Brigo D., Mercurio F., "Interest Rate Models: Theory and Practice with Smile, Inflation and Credit", Springer Finance (2006)

[3] Caligaris O., "Le reti neurali”, Lettera matematica PRISTEM, 61:20-28 (2007)

[4] Caligaris O., Giribone P. G., "Modellizzare la curva dei rendimenti mediante metodologie di apprendimento artificiale: analisi e confronto prestazionale tra le tecniche regressive tradizionali e le reti neurali”, AIFIRM (Italian Association of Financial Industry Risk Management) Magazine, Vol. 10, N.3 (2015)

[5] Caligaris O., Giribone P. G., Ligato S., "Applicazioni delle reti neurali feed-forward per la ricostruzione di superfici di volatilità", AIFIRM (Italian Association of Financial Industry Risk Management) Magazine, Vol. 10, N.1 (2015)

[6] Caligaris O., Giribone P. G., Neffelli M., "Ricostruzione di superfici di volatilità mediante l'utilizzo di reti neurali autoassociative: un caso studio basato sull'analisi non lineare delle component principali", Risk Management Magazine, Vol. 12, N.3 (2017)

[7] Company R., Egorova V. N., Jodar L., Soleymani F., "A local radial basis function method for high-dimensional American option pricing problems", Mathematical Modelling and Analysis, Vol. 23, Issue 1, 117 (2018)

[8] De Prado M. L., "Advances in Financial Machine Learning", Wiley (2018)

[9] Fioribello S., Giribone P. G., Ligato S., "Progettazione di una calibrazione robusta per l'albero stocastico di Hull-White mediante l'implementazione di euristiche globali di ricerca", AIFIRM (Italian Association of Financial Industry Risk Management) Magazine, Vol. 11, N.2 (2016)

[10] Heaton J. B., Polson N., Witte J. H., "Deep Learning for Finance: Deep Portfolios", Applied Stochastic Models in Business and Industry, Vol. 33, Issue 1 (2017)

[11] Jarrow, R., Yildirim Y., "Pricing Treasury Inflation Protected Securities and Related Derivatives using an HJM Model", Journal of Financial and Quantitative Analysis 38(2), 409-430 (2003)

[12] Kazziha, S., "Interest Rate Models, Inflation-based Derivatives, Trigger Notes And Cross-Currency Swaptions", PhD Thesis, Imperial College of Science, Technology and Medicine. London (1999).

[13] Kim P., "MATLAB Deep Learning with Machine learning, Neural Networks and Artificial Intelligence", Apress

[14] Kirby M. J., Miranda, R., "Circular nodes in neural networks", Journal of Neural Computing, 8(2), 390-402 (1996)

[15] Mammadi, S., "Financial time series prediction using artificial neural network based on Levenberg-Marquardt algorithm”, Elsevier, Procedia Computer Science, Vol. 120

[16] Mercurio, F., "Pricing Inflation-Indexed Derivatives", Quantitative Finance, 5-3 (2005)

[17] Pendyala V., "Veracity of Big Data: Machine Learning and other approaches to verifying truthfulness", Apress

[18] Raviola P., Ligato S., Giribone P. G., "Carige Bank integrates a MATLAB based valuation library with its enterprise pricing and risk platform - Case Study", MathWorks User-Story Journal (2013)

[19] Yanui C., Kaijian H., Geoffrey K. F., "Forecasting crude oil prices: a deep learning based model", Elsevier, Procedia Computer Science, Vol. 122

[20] Youngmin, H., "Machine Learning in quantitative finance", Ph.D. Thesis, University of Glasgow (2017) 


\section{Sintesi delle abbreviazioni e delle notazioni}

$C P I=$ Consumer Price Index

$I I S=$ Inflation indexed swap

ZCIIS = Zero-Coupon inflation indexed swap

$Y Y I I S=$ Year-on-Year inflation indexed swap

$I(T)$ o $\mathfrak{J}(T)=$ valore dell'indice inflattivo in corrispondenza dalla scadenza $T$

$P_{n}(t, T)=$ Prezzo di uno Zero-coupon bond al tempo $t$ per la scadenza $T$ nella nominal economy

$P_{r}(t, T)=$ Prezzo di uno Zero-coupon bond al tempo $t$ per la scadenza $T$ nella real economy

$T_{1}, \ldots, T_{i}, \ldots, T_{M}=$ date di pagamento di un $I I S . T_{M}$ coincide con la scadenza del derivato

$\mathfrak{R}_{M}=$ residui mensili standardizzati

$M=$ scadenza dello strumento finanziario (maturity)

$K=$ tasso fisso contrattualizzato per un $I I S$

$N=$ valore nominale

$\varphi_{i}=$ tempo intercorrente tra due date di pagamento, $\left[T_{i-1}, T_{i}\right]$ di un IIS (espresso in frazione d'anno)

$\mathrm{F}_{t}=\sigma$-algebra generata dal processo stocastico di riferimento sino al tempo $t$

$n(t)=$ spot rate istantaneo al tempo $t$ nella nominal economy

$r(t)=$ spot rate istantaneo al tempo $t$ nella real economy

$E(\cdot)=$ valore atteso

$\rho_{i, j}=$ correlazione tra l'asset $i$ e $j$.

$s(x)=$ funzione di attivazione

$\sigma(x)=$ sigmoide

$\tau(x)=$ tangente iperbolica

$\omega=$ peso associato ad un arco della rete neurale

$b=$ bias associato ad un neurone della rete neurale

$u=$ input associato ad un neurone della rete neurale

$\eta=$ segnale processato da un neurone della rete neurale

$\nabla f=$ gradiente della funzione $f$.

$E=$ errore della rete

$o=$ osservazione sperimentale da essere confrontata con l'output della rete neurale

$y=$ output della rete neurale

$\xi=$ segnale d'ingresso associato al primo neurone della coppia

$\eta=$ segnale d'ingresso associato al secondo neurone della coppia

$\delta=$ segnale

$l_{r}=$ learning rate della rete

$\delta=$ contributo da essere propagato all'indietro in accordo con la $\delta$ rule dell'algoritmo di back-propagation.

$t_{j}=$ valore prodotto dalla rete neurale da essere confrontato con quello osservato, $o_{j}$ nell'ambito del supervised learning 


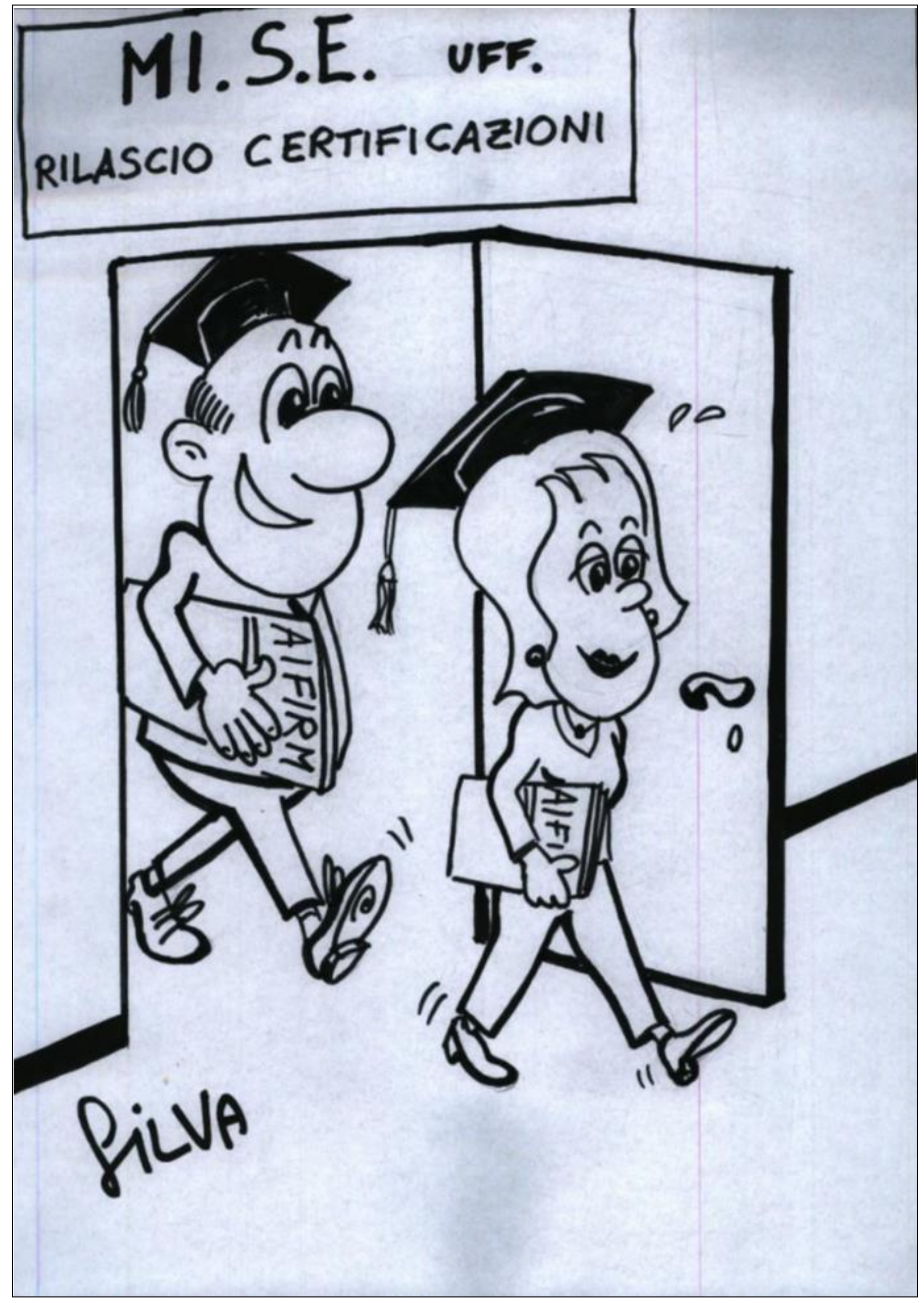

RISK MANAGEMENT MAGAZINE ANNO 13 N 3 - PAGINA - 54 - 
Risk Management Magazine

Anno 13, n 3 Settembre - Dicembre 2018

Direttore Responsabile

Maurizio Vallino

Condirettore

Corrado Meglio

Consiglio Scientifico

Giampaolo Gabbi (Direttore del Consiglio Scientifico),

Ruggero Bertelli,

Paola Bongini,

Anna Bottasso,

Marina Brogi

Ottavio Caligaris,

Simona Cosma,

Paola Ferretti,

Andrea Giacomelli,

Adele Grassi,

Pier Giuseppe Giribone,

Valentina Lagasio,

Duccio Martelli,

Laura Nieri,

Anna Grazia Quaranta,

Pasqualina Porretta,

Francesco Saita,

Enzo Scannella,

Cristiana Schena,

Giuseppe Torluccio

Comitato di redazione

Sara Brianza

Emanuele Diquattro

Fausto Galmarini

Rossano Giuppa

Aldo Letizia

Paolo Palliola

Enzo Rocca

Fabio Salis

Vignettista: Silvano Gaggero

Proprietà, Redazione e Segreteria:

Associazione Italiana Financial Industry Risk Managers (AIFIRM), Via Sile 18, 20139 Milano

Registrazione del Tribunale di Milano $\mathrm{n}^{\circ} 629$ del 10/9/2004

E-mail: segreteria@aifirm.it; Tel. 3896946315 Lunedì - Venerdì h.15-17

Stampa: Algraphy S.n.c. - Passo Ponte Carrega 62-62r, 16141 Genova

Le opinioni espresse negli articoli impegnano unicamente la responsabilità dei rispettivi autori

SPEDIZIONE IN ABBONAMENTO POSTALE AI SOCI AIFIRM RESIDENTI IN ITALIA, IN REGOLA CON L'ISCRIZIONE 


\section{RISK MANAGEMENT MAGAZINE}

Rivista dell'Associazione Italiana Financial Industry Risk Managers

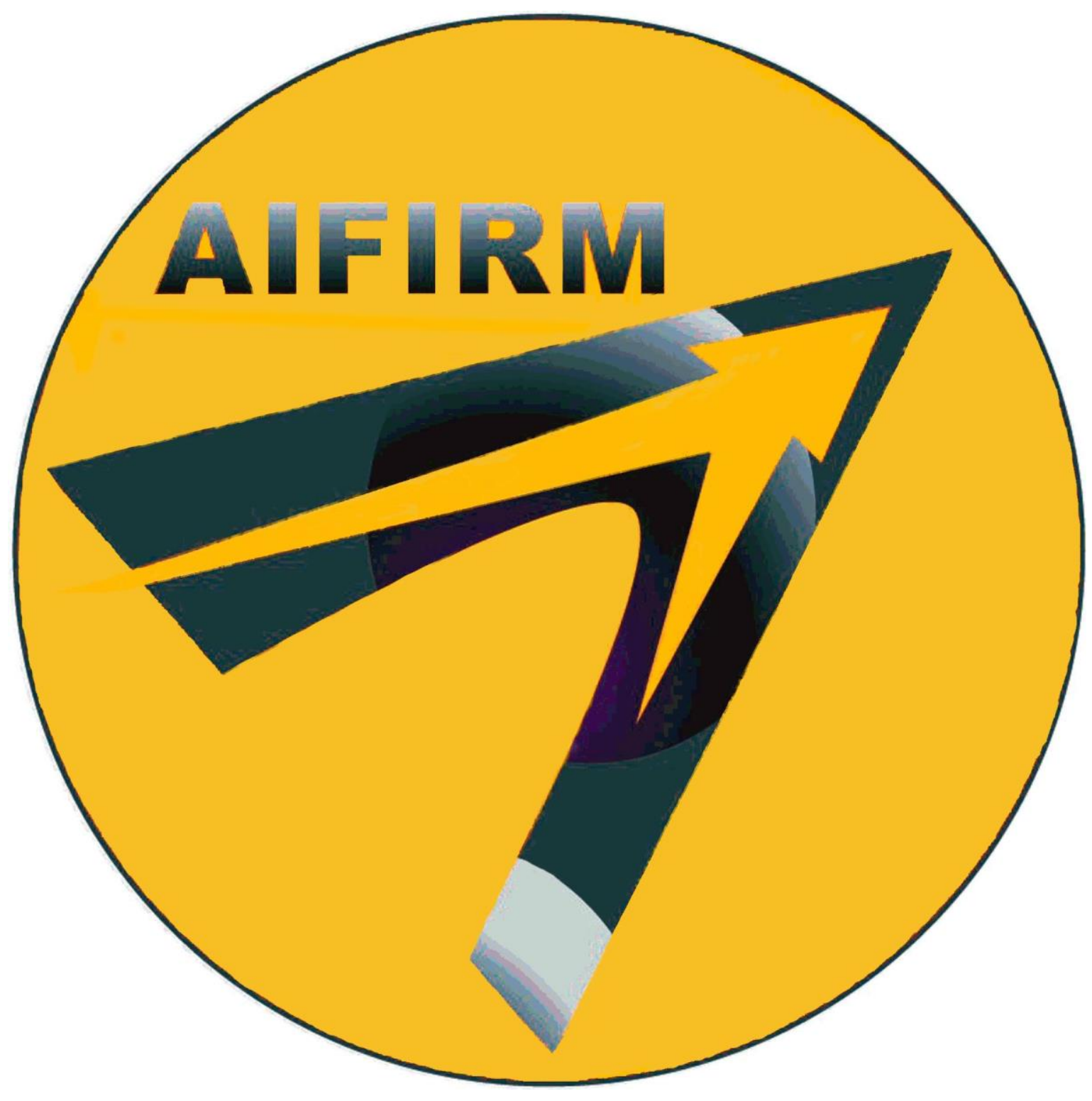

